\title{
Thermal Freeze-Out Parameters and Pseudoentropy from Charged Hadron Spectra in High-Energy Collisions
}

\author{
Xu-Hong Zhang, ${ }^{1}$ Ya-Qin Gao, ${ }^{2}$ Fu-Hu Liu $\mathbb{D},{ }^{1}$ and Khusniddin K. Olimov $\mathbb{D}^{3}$ \\ ${ }^{1}$ Institute of Theoretical Physics, State Key Laboratory of Quantum Optics and Quantum Optics Devices \& Collaborative Innovation \\ Center of Extreme Optics, Shanxi University, Taiyuan 030006, China \\ ${ }^{2}$ Department of Physics, Taiyuan University of Science and Technology, Taiyuan 030024, China \\ ${ }^{3}$ Laboratory of High Energy Physics, Physical-Technical Institute of Uzbekistan Academy of Sciences, Chingiz Aytmatov Str. $2 b$, \\ 100084 Tashkent, Uzbekistan
}

Correspondence should be addressed to Fu-Hu Liu; fuhuliu@163.com and Khusniddin K. Olimov; khkolimov@gmail.com

Received 2 August 2021; Accepted 20 January 2022; Published 21 February 2022

Academic Editor: Carlos Pajares

Copyright (c) $2022 \mathrm{Xu}$-Hong Zhang et al. This is an open access article distributed under the Creative Commons Attribution License, which permits unrestricted use, distribution, and reproduction in any medium, provided the original work is properly cited. The publication of this article was funded by SCOAP S. $^{3}$

\begin{abstract}
We collected the transverse momentum (mass) spectra of charged hadrons $\left(\pi^{-}, \pi^{+}, K^{-}, K^{+}, \bar{p}\right.$, and $\left.p\right)$ produced in collisions over a center-of-mass energy range from 2.70 to $200 \mathrm{GeV}$ (per nucleon pair). The modified Tsallis-Pareto-type function (the TP-like function) with average transverse flow velocity is used to describe the contribution of participant or constituent quarks to transverse momentum of considered hadron. The experimental spectra of $\pi^{\mp}$ and $K^{\mp}$ (or $\bar{p}$ and $p$ ) are fitted by the convolution of two (or three) TP-like functions due to the fact that two (or three) constituent quarks are regarded as two (or three) energy resources in the formation of considered hadron. From the reasonable fits to the spectra, the thermal freeze-out parameters are extracted, and the pseudoentropy is newly defined and extracted. Some parameters quickly change in the energy range of less than $7.7 \mathrm{GeV}$, and slowly change in the energy range of greater than $7.7 \mathrm{GeV}$, indicating the variation of collision mechanism at around $7.7 \mathrm{GeV}$.
\end{abstract}

\section{Introduction}

As the basic gauge field theory [1-3] which is used for describing strong interactions, quantum chromodynamics (QCD) predicts that under the condition of high temperature and high density [4], partons can be released from the confined hadron phase to form a new form of substance that is called quark-gluon plasma (QGP) [5-8]. The hightemperature and high-density fireball formed in relativistic heavy-ion collisions can take the shape of this kind of new form of strongly interacting substance. In the meantime, the particular system composed by this kind of substance is usually described by the QCD phase diagram $[9,10]$. Nevertheless, once the external conditions are changed, the strongly interacting substances described by QCD will undergo a phase transition $[9,10]$. On the one hand, if the thermodynamic properties of the system are expressed by the temperature and the chemical potential of baryons
[11-13], the first-order phase transition will occur in the region where the chemical potential of baryons is higher, and the temperature is lower. On the other hand, in the region where both the baryon chemical potential [11-13] and temperature are high, the system maintains dynamic balance which passes a smooth transition. In other words, there is a critical point [14-19] from hadronic to QGP phases in the deconfinement process, that is the end point of the first-order phase transition of the QCD matter.

One of the most important targets of relativistic heavyion collisions [20-23] is to explore the QCD phase diagram, understand the structure of QGP, and determine the phase boundary $[24,25]$ between different phases. The beam energy scan (BES) project [26-29] started by the Relativistic Heavy Ion Collider (RHIC) in 2010 is to study the phase diagram $[9,10]$ of strongly interacting nuclear matter. The RHIC-BES program [30-32] is performed for mainly three objectives: the first one is to find the onset energy of 


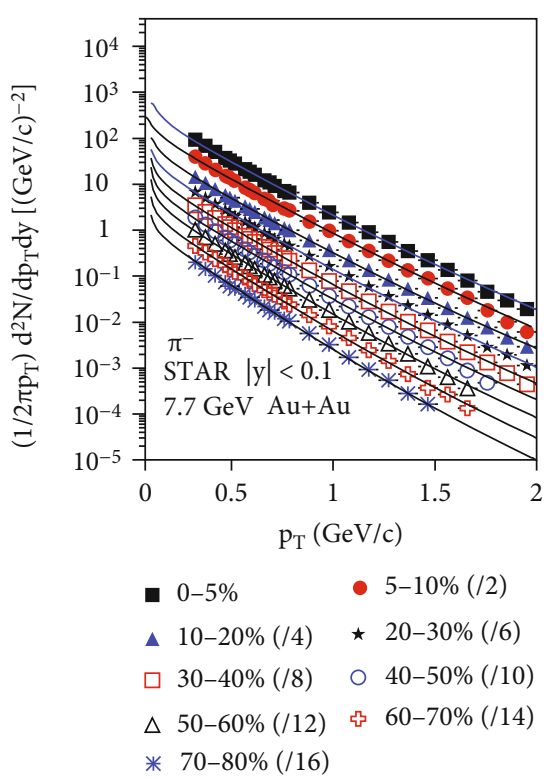

(a)

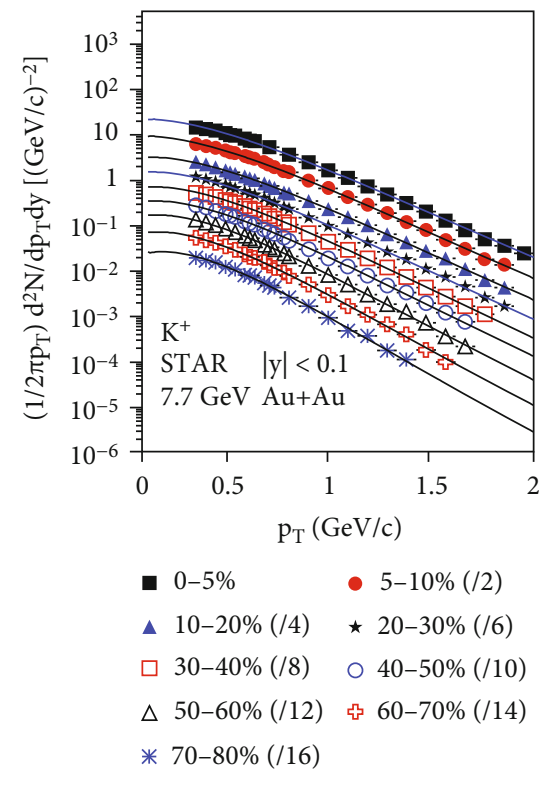

(d)

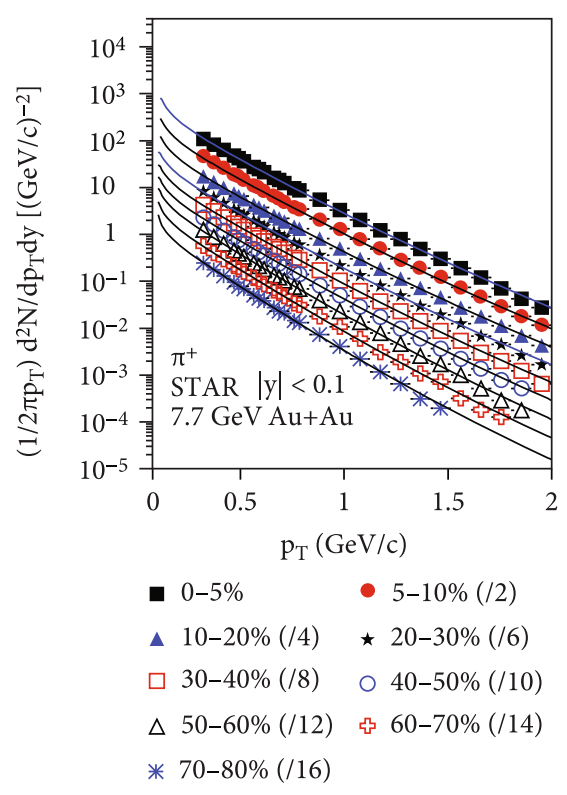

(b)

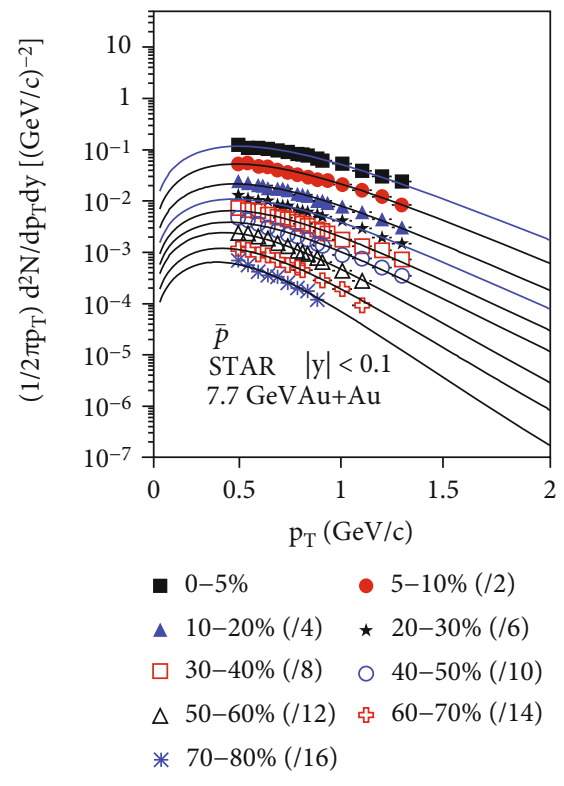

(e)

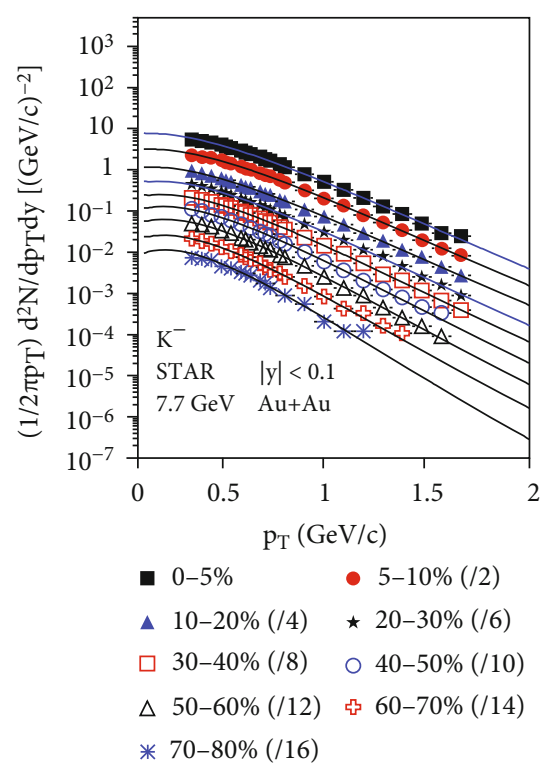

(c)

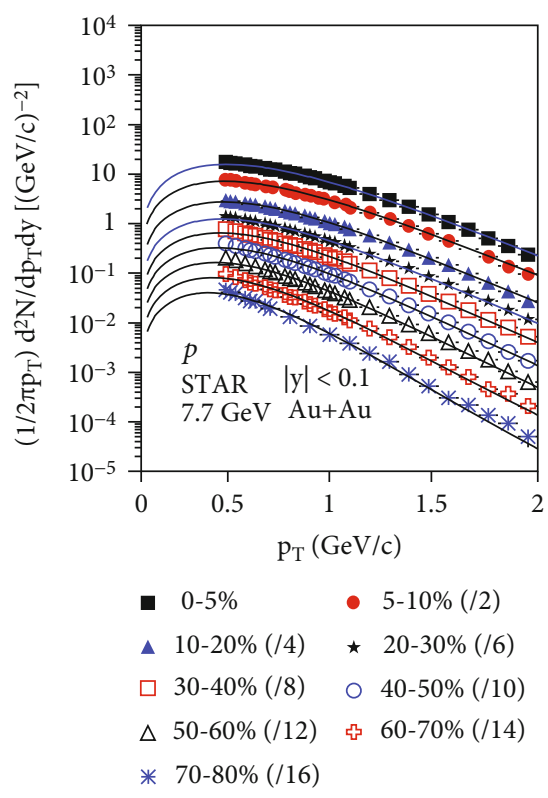

(f)

FIGURE 1: Transverse momentum spectra of $\pi^{-}(\mathrm{a}), \pi^{+}(\mathrm{b}), K^{-}(\mathrm{c}), K^{+}(\mathrm{d}), \bar{p}$ (e), and $p$ (f) produced in $7.7 \mathrm{GeV}$ Au-Au collisions with various centrality intervals and at the midrapidity. The symbols represent the experimental data measured by the STAR Collaboration in the RHICBES program $[33,34]$ and rescaled by different amounts marked in the panels. The curves are our results fitted by Equation (4) for $\pi^{\mp}$ and $K^{\mp}$ (panels (a)-(d)) or Equation (6) for $\bar{p}$ and $p$ (panels (e) and (f)).

deconfinement phase transition $[9,10]$. The second one is to identify the critical point of the QCD phase diagram $[9,10$, 14-17]. And the third one is to determine the characteristics of the first-order phase transition. Since the baryon chemical potential of matter is related to the collision energy [11-13], researchers expect to find the corresponding critical point of phase transition by the way of changing the collision energy. The RHIC-BES program including its fixed target experiments is able to vary the collision energy over a wide range in low energy region, thereby achieving an extensive range of baryon chemical potential, further expanding the search objective.

Hadrons are particles involved in strong interactions, including two kinds of particles: mesons (bosons) and baryons (fermions). The most common mesons measured in experiments include, but are not limited to, negatively and positively charged $\pi$ mesons $\left(\pi^{-}\right.$and $\left.\pi^{+}\right)$and negatively and positively charged $K$ mesons $\left(K^{-}\right.$and $\left.K^{+}\right)$. The most common baryons measured in experiments include, but are not limited to, antiprotons and protons $(\bar{p}$ and $p)$. In 
TABLe 1: Values of $n, T_{0}, a_{0},\left\langle\beta_{t}\right\rangle, \chi^{2}$, and ndof corresponding to the curves in Figure 1.

\begin{tabular}{|c|c|c|c|c|c|c|c|c|}
\hline Figure & Particle & $\sqrt{s_{N N}}(\mathrm{GeV})$ & Selection & $n$ & $T_{0}(\mathrm{GeV})$ & $a_{0}$ & $\left\langle\beta_{t}\right\rangle(c)$ & $\chi^{2} /$ ndof \\
\hline \multirow[t]{9}{*}{ Figure 1(a) } & $\pi^{-}$ & 7.7 & $0-5 \%$ & $17.8 \pm 0.7$ & $0.165 \pm 0.003$ & $-0.396 \pm 0.005$ & $0.112 \pm 0.003$ & $14 / 21$ \\
\hline & & $|y|<0.1$ & $5-10 \%$ & $16.5 \pm 0.7$ & $0.158 \pm 0.003$ & $-0.383 \pm 0.005$ & $0.109 \pm 0.003$ & $27 / 21$ \\
\hline & & & $10-20 \%$ & $15.0 \pm 0.5$ & $0.155 \pm 0.003$ & $-0.379 \pm 0.005$ & $0.106 \pm 0.003$ & $8 / 21$ \\
\hline & & & $20-30 \%$ & $14.9 \pm 0.5$ & $0.150 \pm 0.003$ & $-0.374 \pm 0.005$ & $0.102 \pm 0.003$ & $14 / 21$ \\
\hline & & & $30-40 \%$ & $14.6 \pm 0.5$ & $0.147 \pm 0.002$ & $-0.369 \pm 0.004$ & $0.099 \pm 0.002$ & $18 / 21$ \\
\hline & & & $40-50 \%$ & $14.4 \pm 0.5$ & $0.143 \pm 0.002$ & $-0.365 \pm 0.004$ & $0.098 \pm 0.002$ & $15 / 19$ \\
\hline & & & $50-60 \%$ & $14.0 \pm 0.4$ & $0.135 \pm 0.002$ & $-0.362 \pm 0.004$ & $0.098 \pm 0.002$ & $10 / 18$ \\
\hline & & & $60-70 \%$ & $13.8 \pm 0.4$ & $0.128 \pm 0.002$ & $-0.358 \pm 0.004$ & $0.094 \pm 0.002$ & $17 / 18$ \\
\hline & & & $70-80 \%$ & $13.7 \pm 0.4$ & $0.121 \pm 0.002$ & $-0.356 \pm 0.004$ & $0.093 \pm 0.002$ & $6 / 16$ \\
\hline \multirow[t]{9}{*}{ Figure 1(b) } & $\pi^{+}$ & 7.7 & $0-5 \%$ & $18.2 \pm 0.8$ & $0.171 \pm 0.003$ & $-0.420 \pm 0.005$ & $0.119 \pm 0.003$ & $12 / 21$ \\
\hline & & $|y|<0.1$ & $5-10 \%$ & $17.3 \pm 0.7$ & $0.168 \pm 0.003$ & $-0.417 \pm 0.005$ & $0.116 \pm 0.003$ & $5 / 21$ \\
\hline & & & $10-20 \%$ & $16.0 \pm 0.6$ & $0.164 \pm 0.003$ & $-0.411 \pm 0.005$ & $0.114 \pm 0.003$ & $5 / 21$ \\
\hline & & & $20-30 \%$ & $15.7 \pm 0.6$ & $0.160 \pm 0.003$ & $-0.400 \pm 0.005$ & $0.111 \pm 0.003$ & $9 / 21$ \\
\hline & & & $30-40 \%$ & $15.3 \pm 0.6$ & $0.154 \pm 0.003$ & $-0.398 \pm 0.005$ & $0.109 \pm 0.003$ & $12 / 21$ \\
\hline & & & $40-50 \%$ & $14.9 \pm 0.5$ & $0.147 \pm 0.002$ & $-0.390 \pm 0.005$ & $0.106 \pm 0.003$ & $6 / 20$ \\
\hline & & & $50-60 \%$ & $14.4 \pm 0.5$ & $0.138 \pm 0.002$ & $-0.385 \pm 0.005$ & $0.104 \pm 0.003$ & $15 / 20$ \\
\hline & & & $60-70 \%$ & $13.9 \pm 0.4$ & $0.133 \pm 0.002$ & $-0.377 \pm 0.005$ & $0.102 \pm 0.003$ & $13 / 19$ \\
\hline & & & $70-80 \%$ & $13.4 \pm 0.4$ & $0.126 \pm 0.002$ & $-0.370 \pm 0.005$ & $0.100 \pm 0.002$ & $10 / 16$ \\
\hline \multirow[t]{9}{*}{ Figure 1(c) } & $K^{-}$ & 7.7 & $0-5 \%$ & $27.9 \pm 1.7$ & $0.147 \pm 0.002$ & $0.011 \pm 0.004$ & $0.106 \pm 0.005$ & $9 / 18$ \\
\hline & & $|y|<0.1$ & $5-10 \%$ & $24.8 \pm 1.4$ & $0.143 \pm 0.002$ & $0.018 \pm 0.004$ & $0.105 \pm 0.005$ & $3 / 18$ \\
\hline & & & $10-20 \%$ & $22.8 \pm 1.2$ & $0.139 \pm 0.002$ & $0.025 \pm 0.004$ & $0.103 \pm 0.005$ & $5 / 18$ \\
\hline & & & $20-30 \%$ & $21.8 \pm 1.1$ & $0.129 \pm 0.002$ & $0.045 \pm 0.005$ & $0.102 \pm 0.005$ & $8 / 18$ \\
\hline & & & $30-40 \%$ & $21.5 \pm 1.1$ & $0.124 \pm 0.002$ & $0.061 \pm 0.005$ & $0.101 \pm 0.005$ & $5 / 18$ \\
\hline & & & $40-50 \%$ & $20.8 \pm 1.0$ & $0.115 \pm 0.002$ & $0.070 \pm 0.005$ & $0.096 \pm 0.004$ & $11 / 15$ \\
\hline & & & $50-60 \%$ & $20.6 \pm 1.0$ & $0.107 \pm 0.002$ & $0.081 \pm 0.005$ & $0.096 \pm 0.004$ & $5 / 16$ \\
\hline & & & $60-70 \%$ & $18.9 \pm 0.8$ & $0.100 \pm 0.002$ & $0.085 \pm 0.005$ & $0.094 \pm 0.004$ & $11 / 14$ \\
\hline & & & $70-80 \%$ & $18.2 \pm 0.8$ & $0.089 \pm 0.002$ & $0.093 \pm 0.005$ & $0.092 \pm 0.004$ & $26 / 11$ \\
\hline \multirow[t]{9}{*}{ Figure $1(\mathrm{~d})$} & $K^{+}$ & 7.7 & $0-5 \%$ & $29.1 \pm 1.9$ & $0.164 \pm 0.003$ & $-0.022 \pm 0.004$ & $0.124 \pm 0.005$ & $9 / 18$ \\
\hline & & $|y|<0.1$ & $5-10 \%$ & $27.2 \pm 1.7$ & $0.160 \pm 0.003$ & $-0.020 \pm 0.004$ & $0.112 \pm 0.005$ & $6 / 20$ \\
\hline & & & $10-20 \%$ & $25.7 \pm 1.5$ & $0.155 \pm 0.003$ & $0.001 \pm 0.004$ & $0.109 \pm 0.005$ & $6 / 20$ \\
\hline & & & $20-30 \%$ & $25.1 \pm 1.5$ & $0.149 \pm 0.002$ & $0.005 \pm 0.004$ & $0.105 \pm 0.005$ & $5 / 20$ \\
\hline & & & $30-40 \%$ & $24.5 \pm 1.4$ & $0.144 \pm 0.002$ & $0.014 \pm 0.004$ & $0.099 \pm 0.004$ & $4 / 19$ \\
\hline & & & $40-50 \%$ & $22.5 \pm 1.2$ & $0.139 \pm 0.002$ & $0.019 \pm 0.004$ & $0.091 \pm 0.004$ & $9 / 18$ \\
\hline & & & $50-60 \%$ & $21.9 \pm 1.1$ & $0.129 \pm 0.002$ & $0.027 \pm 0.004$ & $0.085 \pm 0.004$ & $7 / 17$ \\
\hline & & & $60-70 \%$ & $20.1 \pm 1.0$ & $0.120 \pm 0.002$ & $0.034 \pm 0.005$ & $0.082 \pm 0.004$ & $8 / 16$ \\
\hline & & & $70-80 \%$ & $18.7 \pm 0.8$ & $0.112 \pm 0.002$ & $0.049 \pm 0.005$ & $0.079 \pm 0.004$ & $9 / 13$ \\
\hline
\end{tabular}


TABLE 1: Continued.

\begin{tabular}{|c|c|c|c|c|c|c|c|c|}
\hline Figure & Particle & $\sqrt{s_{N N}}(\mathrm{GeV})$ & Selection & $n$ & $T_{0}(\mathrm{GeV})$ & $a_{0}$ & $\left\langle\beta_{t}\right\rangle(c)$ & $\chi^{2} /$ ndof \\
\hline \multirow[t]{9}{*}{ Figure 1(e) } & $\bar{p}$ & 7.7 & $0-5 \%$ & $16.6 \pm 0.7$ & $0.128 \pm 0.002$ & $0.006 \pm 0.004$ & $0.347 \pm 0.008$ & $2 / 10$ \\
\hline & & $|y|<0.1$ & $5-10 \%$ & $15.8 \pm 0.6$ & $0.123 \pm 0.002$ & $0.007 \pm 0.004$ & $0.336 \pm 0.008$ & $4 / 9$ \\
\hline & & & $10-20 \%$ & $15.1 \pm 0.6$ & $0.116 \pm 0.002$ & $0.011 \pm 0.004$ & $0.325 \pm 0.008$ & $5 / 13$ \\
\hline & & & $20-30 \%$ & $14.6 \pm 0.5$ & $0.113 \pm 0.002$ & $0.015 \pm 0.004$ & $0.317 \pm 0.008$ & $5 / 11$ \\
\hline & & & $30-40 \%$ & $13.6 \pm 0.4$ & $0.104 \pm 0.002$ & $0.017 \pm 0.004$ & $0.309 \pm 0.008$ & $6 / 12$ \\
\hline & & & $40-50 \%$ & $12.8 \pm 0.3$ & $0.098 \pm 0.002$ & $0.020 \pm 0.004$ & $0.291 \pm 0.007$ & $11 / 9$ \\
\hline & & & $50-60 \%$ & $12.5 \pm 0.3$ & $0.086 \pm 0.001$ & $0.020 \pm 0.004$ & $0.276 \pm 0.007$ & $1 / 8$ \\
\hline & & & $60-70 \%$ & $11.9 \pm 0.3$ & $0.079 \pm 0.001$ & $0.023 \pm 0.004$ & $0.271 \pm 0.007$ & $2 / 6$ \\
\hline & & & $70-80 \%$ & $11.2 \pm 0.3$ & $0.069 \pm 0.001$ & $0.024 \pm 0.004$ & $0.260 \pm 0.007$ & $2 / 4$ \\
\hline \multirow[t]{9}{*}{ Figure 1(f) } & $p$ & 7.7 & $0-5 \%$ & $18.4 \pm 0.8$ & $0.129 \pm 0.002$ & $0.006 \pm 0.004$ & $0.346 \pm 0.008$ & $5 / 24$ \\
\hline & & $|y|<0.1$ & $5-10 \%$ & $17.6 \pm 0.7$ & $0.127 \pm 0.002$ & $0.011 \pm 0.004$ & $0.334 \pm 0.008$ & $4 / 24$ \\
\hline & & & $10-20 \%$ & $16.7 \pm 0.7$ & $0.120 \pm 0.002$ & $0.016 \pm 0.004$ & $0.321 \pm 0.008$ & $4 / 24$ \\
\hline & & & $20-30 \%$ & $16.4 \pm 0.7$ & $0.117 \pm 0.002$ & $0.017 \pm 0.004$ & $0.314 \pm 0.008$ & $5 / 24$ \\
\hline & & & $30-40 \%$ & $15.0 \pm 0.5$ & $0.112 \pm 0.002$ & $0.019 \pm 0.004$ & $0.304 \pm 0.008$ & $9 / 23$ \\
\hline & & & $40-50 \%$ & $14.6 \pm 0.5$ & $0.105 \pm 0.002$ & $0.021 \pm 0.004$ & $0.298 \pm 0.007$ & $11 / 23$ \\
\hline & & & $50-60 \%$ & $13.4 \pm 0.4$ & $0.100 \pm 0.002$ & $0.023 \pm 0.004$ & $0.282 \pm 0.007$ & $16 / 22$ \\
\hline & & & $60-70 \%$ & $12.5 \pm 0.3$ & $0.091 \pm 0.001$ & $0.025 \pm 0.004$ & $0.271 \pm 0.007$ & $20 / 13$ \\
\hline & & & $70-80 \%$ & $11.3 \pm 0.3$ & $0.079 \pm 0.001$ & $0.028 \pm 0.004$ & $0.260 \pm 0.007$ & $25 / 16$ \\
\hline
\end{tabular}

the RHIC-BES program and at the previous and lower energy such as the Alternating Gradient Synchrotron (AGS) energy, $\pi^{-}, \pi^{+}, K^{-}, K^{+}, \bar{p}$, and $p$ are particularly important. In fact, the abundant transverse momentum (mass) spectra of the mentioned charged hadrons can be used to extract the common thermal parameters such as the freeze-out temperature and transverse flow velocity. Meanwhile, the novel pseudoentropy, which will be defined later in this paper, can be also extracted from the spectra.

In this paper, the transverse momentum spectra of $\pi^{-}$, $\pi^{+}, K^{-}, K^{+}, \bar{p}$, and $p$ produced at midrapidity (mid- $y$ ) in gold-gold $(\mathrm{Au}-\mathrm{Au})$ collisions with different centralities at the RHIC and its BES energy [33-35] are collected. Meanwhile, the transverse mass spectra of the mentioned charged hadrons produced at mid- $y$ in central $\mathrm{Au}-\mathrm{Au}$ collisions at the AGS energy [36-38] are also collected, though the spectra of $\bar{p}$ are not available due to low energy. These spectra are used to extract the thermal freeze-out parameters and the pseudoentropy.

The remainder of this paper is structured as follows. The formalism and method are briefly introduced in Section 2. The results and discussion are given in Section 3. In Section 4 , we summarize our main observations and conclusions.

\section{Formalism and Method}

Nonextensive thermodynamics is a new method for studying heavy-ion collisions at relativistic energy. In the collisions such as in Au-Au collisions, the Tsallis-Pareto-type function [39-44] can fit the transverse momentum $\left(p_{T}\right)$ spectra in low and intermediate regions, particularly in the final-state or hadronization process, demonstrating a strong relation among particles. However, in very low $-p_{T}$ region, the fit result is not ideal, and the meaning of Tsallis parameters remains an open question.

In order to better fit the spectra in very low- $p_{T}$ region, we express the information contained in the parameter more intuitively using the modified Tsallis-Pareto-type function (the TP-like function) as follows $[45,46]$ :

$$
f_{p_{T}}\left(p_{T}\right)=\frac{1}{N} \frac{d N}{d p_{T}}=C p_{T}^{a_{0}}\left(1+\frac{m_{T}-m_{0}}{n T}\right)^{-n} .
$$

Here, $N$ is the number of particles, $C$ is the normalization constant, $m_{0}$ is the rest mass of the considered particle, $n$ is the power index that describes the degree of nonequilibrium, $T$ is the effective temperature of the collision system, $a_{0}$ is the correction index, and $m_{T}=\sqrt{p_{T}^{2}+m_{0}^{2}}$ is the transverse mass of the particle. As an extention, the TP-like function is naturally converged to the Tsallis-Pareto-type function if we set $a_{0}=1$ in Equation (1).

In order to obtain the thermal or kinetic (or kinematic) freeze-out temperature $T_{0}$ and the average transverse flow velocity $\left\langle\beta_{t}\right\rangle[47,48]$, one may fit firstly the $p_{T}$ spectra of particles to obtain the effective temperature $T$. The average $p_{T}\left(\left\langle p_{T}\right\rangle\right)$ and average energy $(\bar{m})$ of particles in the source rest frame can be obtained by using the Monte Carlo algorithm. Then, one may extract the intercept as $T_{0}$ in the linear relation of $T$ versus $m_{0}$ and further obtain the slope as $\left\langle\beta_{t}\right\rangle$ in the linear relation of $\left\langle p_{T}\right\rangle$ versus $\bar{m}$. 


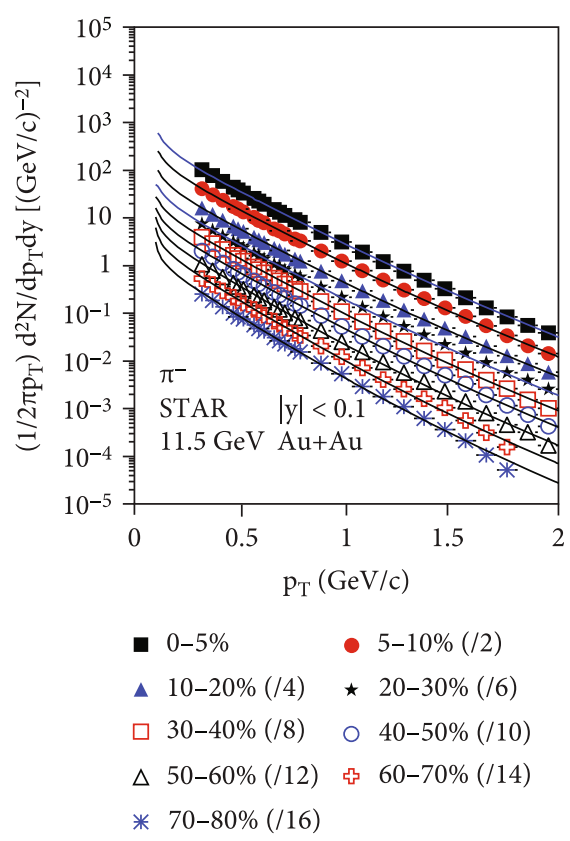

(a)

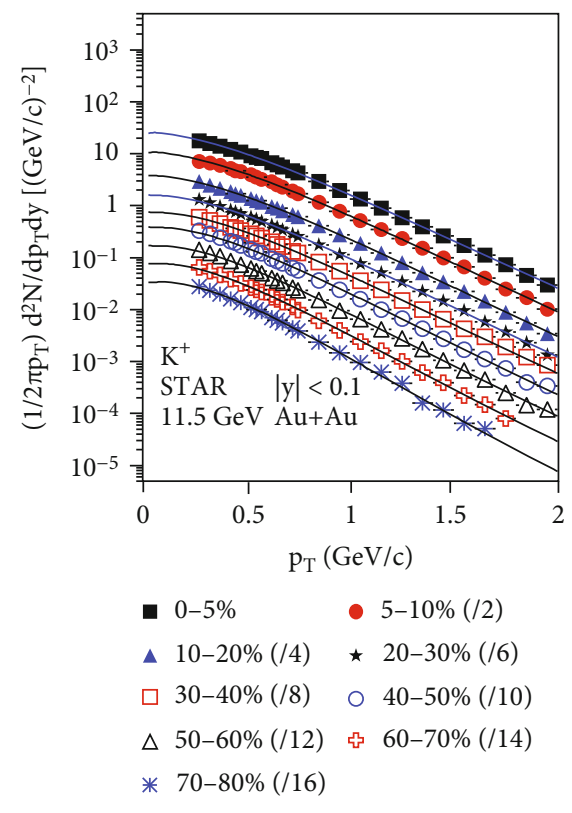

(d)

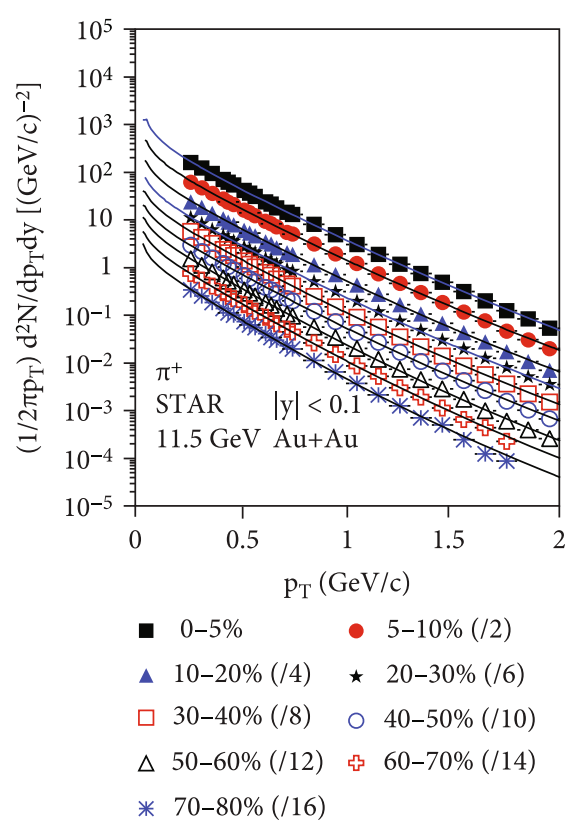

(b)

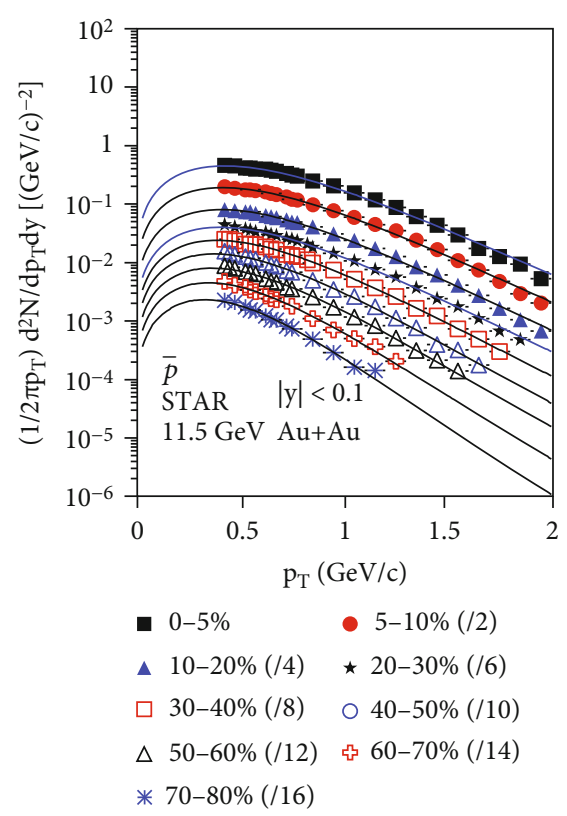

(e)

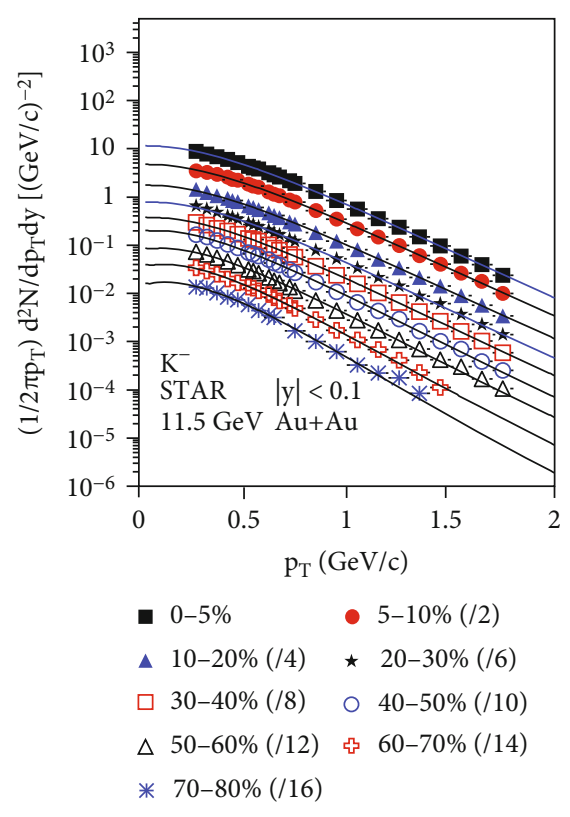

(c)

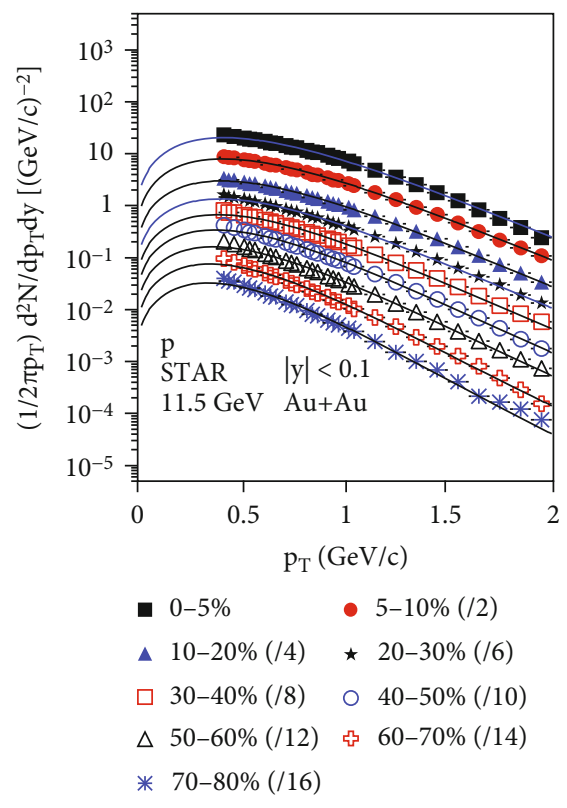

(f)

FIgURE 2: Same as Figure 1, but showing the results for $11.5 \mathrm{GeV}$ Au-Au collisions. The symbols represent the STAR data [33].

For purpose of simplifying this complicated solution process, based on the idea of other work [49], we modify $m_{T}$ and $p_{T}$ in Equation (1). For clarity, we use $m_{T}^{\prime}$ and $p^{\prime}{ }_{T}$ instead of $m_{T}$ and $p_{T}$ in Equation (1) respectively. The Lorentz-like transformation is $m_{T}^{\prime}=\left\langle\gamma_{t}\right\rangle\left(m_{T}-p_{T}\left\langle\beta_{t}\right\rangle\right)$ and $\left|p^{\prime}{ }_{T}\right|=\left\langle\gamma_{t}\right\rangle\left|p_{T}-m_{T}\left\langle\beta_{t}\right\rangle\right|$, where $\left\langle\gamma_{t}\right\rangle=1 / \sqrt{1-\left\langle\beta_{t}\right\rangle^{2}}$ is the Lorentz-like factor. The absolute value $\left|p_{T}-m_{T}\left\langle\beta_{t}\right\rangle\right|$ is used due to the fact that $p_{T}^{\prime}$ is positive and $p_{T}-m_{T}\left\langle\beta_{t}\right\rangle$ is possibly negative in low- $p_{T}$ region. After the conversion, the new TP-like function $f\left(p_{T}\right)$ certainly obeys the relation
$f_{p_{T}^{\prime}}\left(p_{T}^{\prime}\right)\left|d p_{T}^{\prime}\right|=f\left(p_{T}\right)\left|d p_{T}\right|$. We have

$$
\begin{aligned}
f\left(p_{T}\right)= & C \frac{\left\langle\gamma_{t}\right\rangle^{a_{0}+1}}{m_{T}}\left(m_{T}-p_{T}\left\langle\beta_{t}\right\rangle\right)\left|p_{T}-m_{T}\left\langle\beta_{t}\right\rangle\right|^{a_{0}} \\
& \times\left[1+\frac{\left\langle\gamma_{t}\right\rangle\left(m_{T}-p_{T}\left\langle\beta_{t}\right\rangle\right)-m_{0}}{n T_{0}}\right]^{-n} .
\end{aligned}
$$

One can see that $T$ in Equation (1) is naturally converted to $T_{0}$ in Equation (2). 


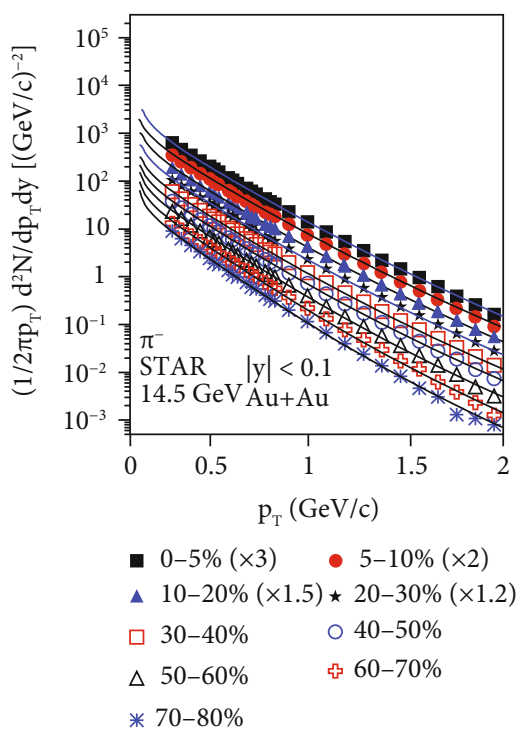

(a)

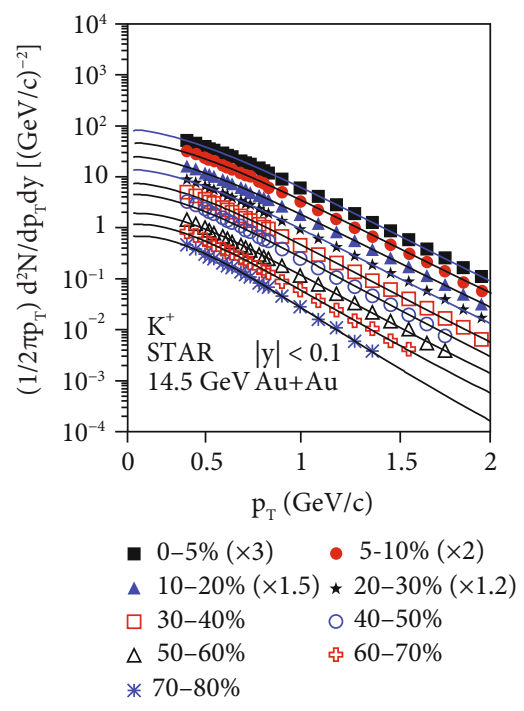

(d)

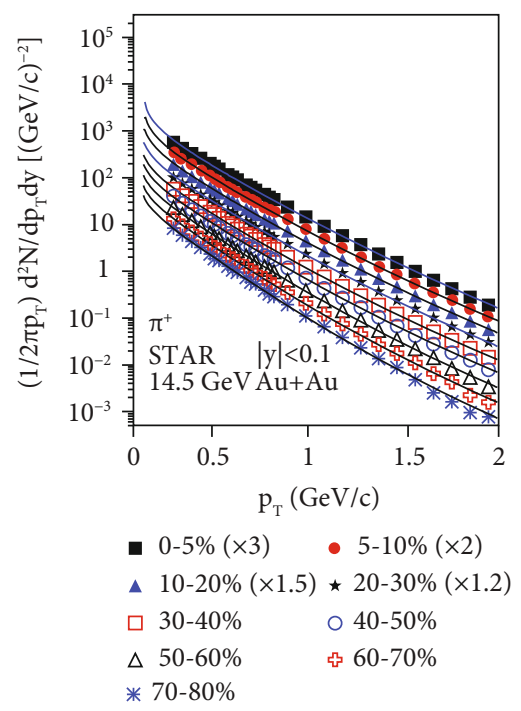

(b)

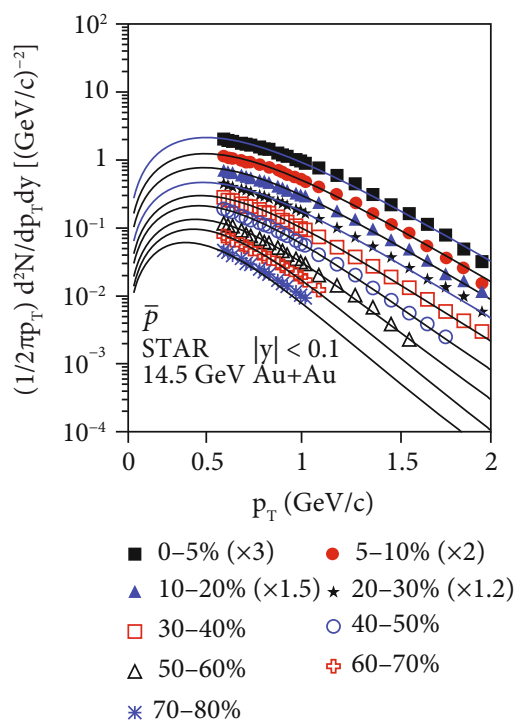

(e)

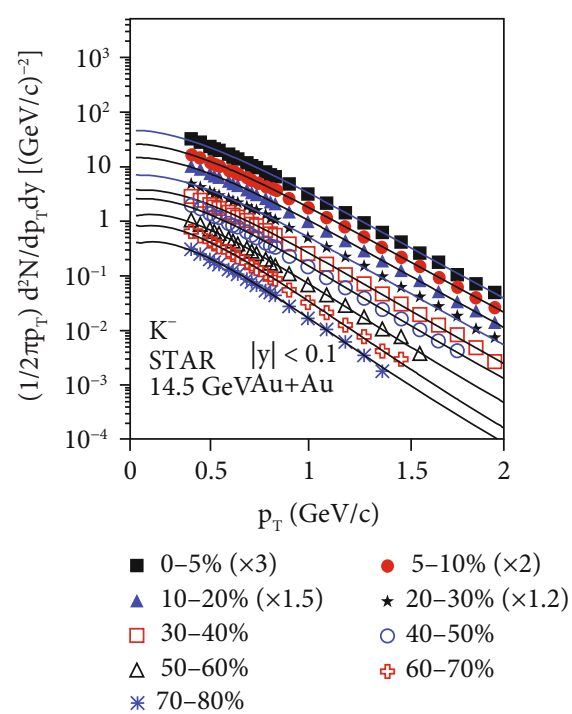

(c)

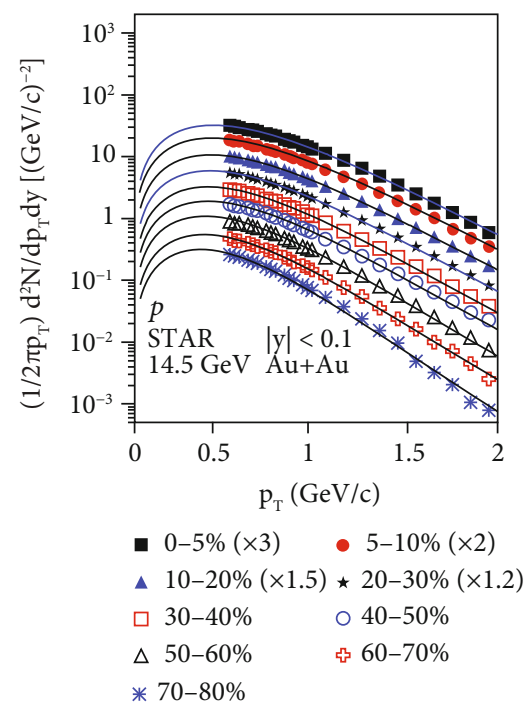

(f)

FIGURE 3: Same as Figure 1, but showing the results for $14.5 \mathrm{GeV} \mathrm{Au-Au} \mathrm{collisions.} \mathrm{The} \mathrm{symbols} \mathrm{represent} \mathrm{the} \mathrm{STAR} \mathrm{data} \mathrm{[34].}$

Our exploratory research shows that although Equation (2) is applicable in the fit of $p_{T}$ spectra, it is not flexible in some cases and explicable in depth at quark level due to $m_{0}$ being the rest mass of the considered particle. Empirically, Equation (2) can be regarded as the probability density function obeyed by the transverse momentum $p_{t i}$ of the $i$-th constituent quark that contributes to $p_{T}$ of particle. Concretely, we have new TP-like function to be

$$
\begin{aligned}
f_{i}\left(p_{t i}\right)= & C_{i} \frac{\left\langle\gamma_{t}\right\rangle^{a_{0}+1}}{m_{t i}}\left(m_{t i}-p_{t i}\left\langle\beta_{t}\right\rangle\right)\left|p_{t i}-m_{t i}\left\langle\beta_{t}\right\rangle\right|^{a_{0}} \\
& \times\left[1+\frac{\left\langle\gamma_{t}\right\rangle\left(m_{t i}-p_{t i}\left\langle\beta_{t}\right\rangle\right)-m_{0 i}}{n T_{0}}\right]^{-n}
\end{aligned}
$$

where $m_{0 i}$ is the constituent mass $\left(0.31 \mathrm{MeV} / c^{2}\right.$ for $u$ and $d$ quarks and $0.5 \mathrm{GeV} / c^{2}$ for $s$ quark, as given in general textbook [50]), and $m_{t i}=\sqrt{p_{t i}^{2}+m_{0 i}^{2}}$ is the transverse mass of the $i$-th quark.

For a meson, $p_{T}=p_{t 1}+p_{t 2}$ due to two constituent quarks which contribute independently. The probability density function of meson's $p_{T}$ is the convolution of two TP-like functions. For a baryon, $p_{T}=p_{t 1}+p_{t 2}+p_{t 3}$ due to three constituent quarks which also contribute independently. The probability density function of baryon's $p_{T}$ is the convolution of three TP-like functions. This analysis is at the quark level due to the fact that it is based on the constituent mass of quark. The present method is similar to the analysis at the particle level in terms of the similar convolution. 


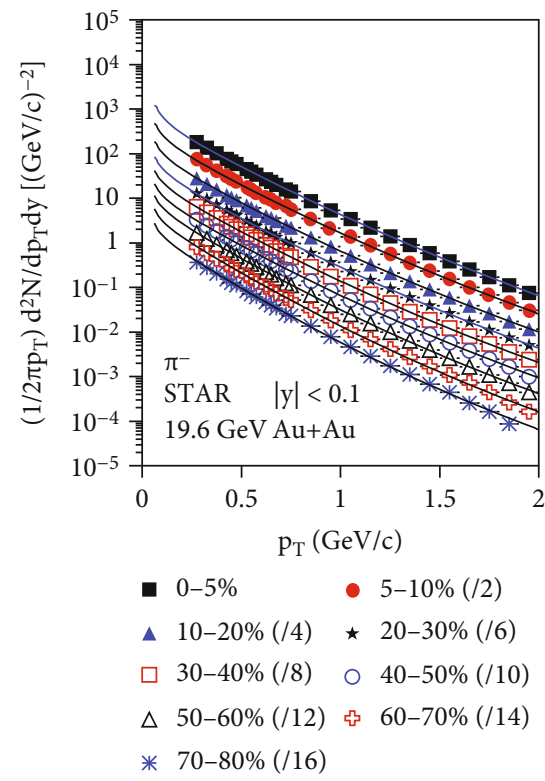

(a)

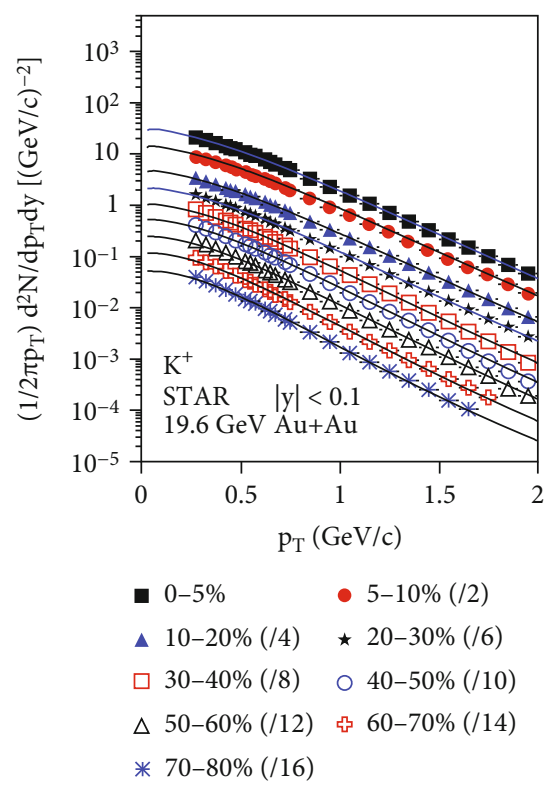

(d)

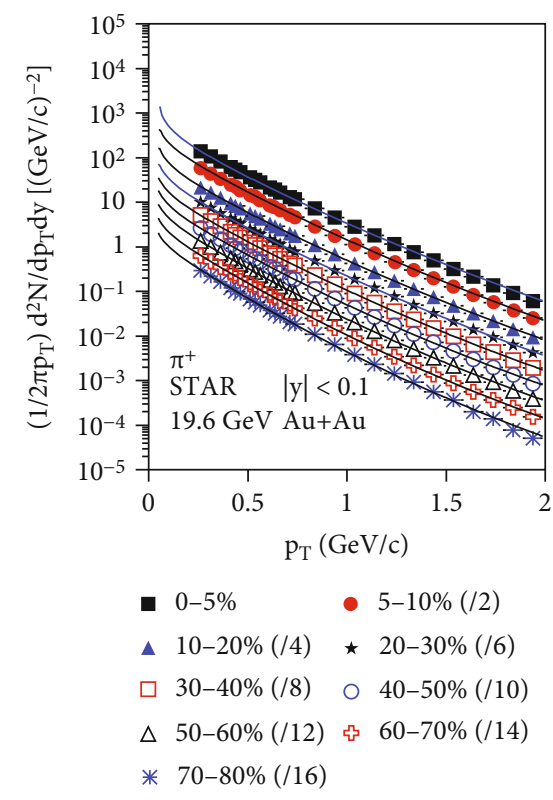

(b)

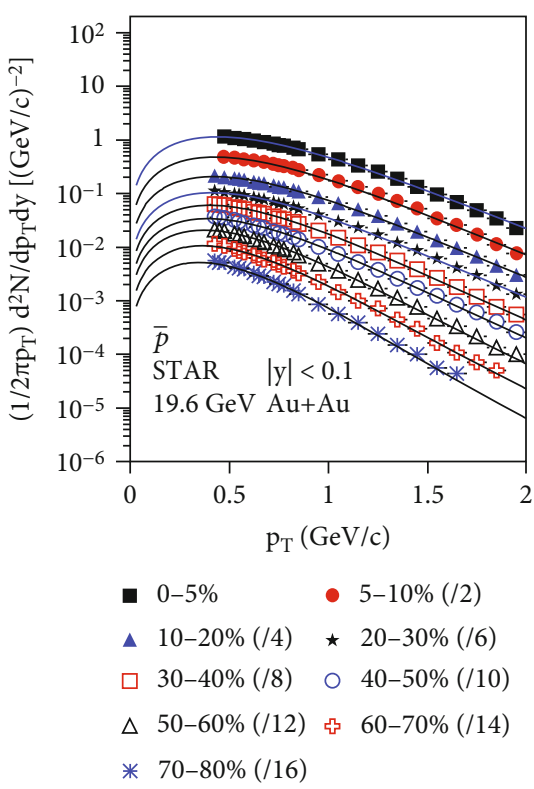

(e)

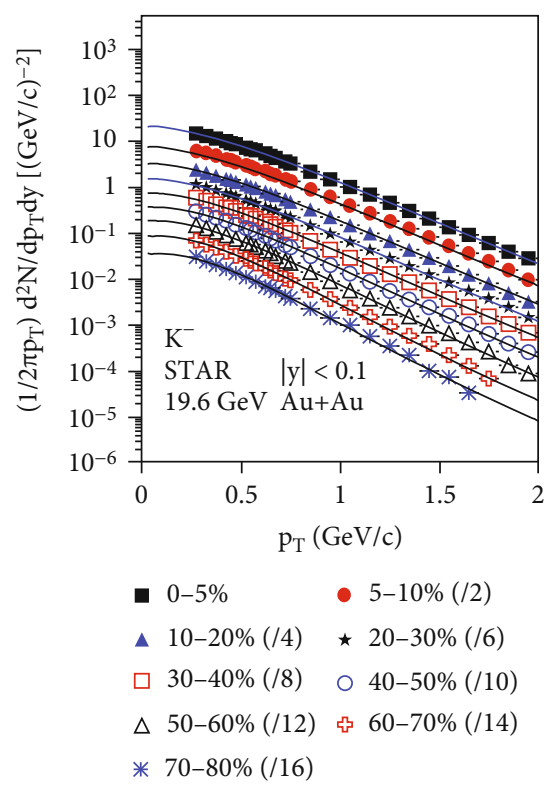

(c)

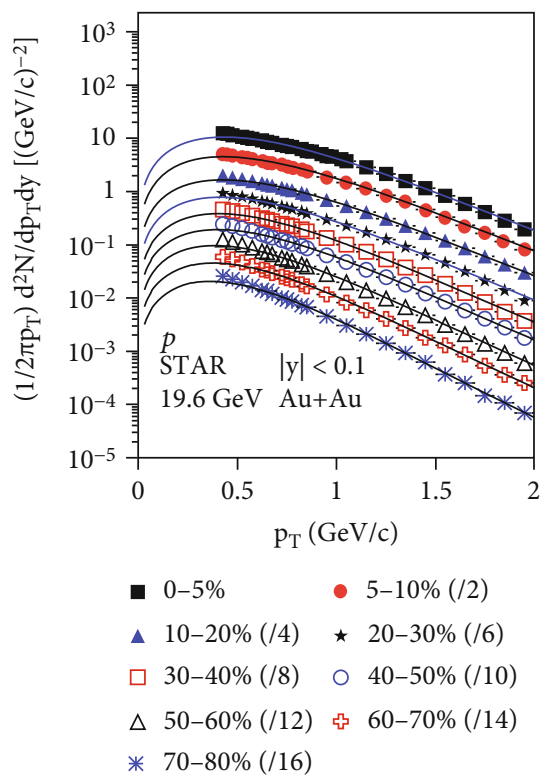

(f)

FIgURE 4: Same as Figure 1, but showing the results for $19.6 \mathrm{GeV} \mathrm{Au-Au} \mathrm{collisions.} \mathrm{The} \mathrm{symbols} \mathrm{represent} \mathrm{the} \mathrm{STAR} \mathrm{data} \mathrm{[33].}$

For mesons, we have the convolution of two TP-like functions to be

$$
\begin{aligned}
f\left(p_{T}\right) & =\int_{0}^{p_{T}} f_{1}\left(p_{t 1}\right) f_{2}\left(p_{T}-p_{t 1}\right) d p_{t 1} \\
& =\int_{0}^{p_{T}} f_{2}\left(p_{t 2}\right) f_{1}\left(p_{T}-p_{t 2}\right) d p_{t 2} .
\end{aligned}
$$

For baryons, we have the convolution of the first two TPlike functions to be

$$
\begin{aligned}
f_{12}\left(p_{t 12}\right) & =\int_{0}^{p_{t 12}} f_{1}\left(p_{t 1}\right) f_{2}\left(p_{t 12}-p_{t 1}\right) d p_{t 1} \\
& =\int_{0}^{p_{t 12}} f_{2}\left(p_{t 2}\right) f_{1}\left(p_{t 12}-p_{t 2}\right) d p_{t 2} .
\end{aligned}
$$

The convolution of three TP-like functions is

$$
\begin{aligned}
f\left(p_{T}\right) & =\int_{0}^{p_{T}} f_{12}\left(p_{t 12}\right) f_{3}\left(p_{T}-p_{t 12}\right) d p_{t 12} \\
& =\int_{0}^{p_{T}} f_{3}\left(p_{t 3}\right) f_{12}\left(p_{T}-p_{t 3}\right) d p_{t 3}
\end{aligned}
$$




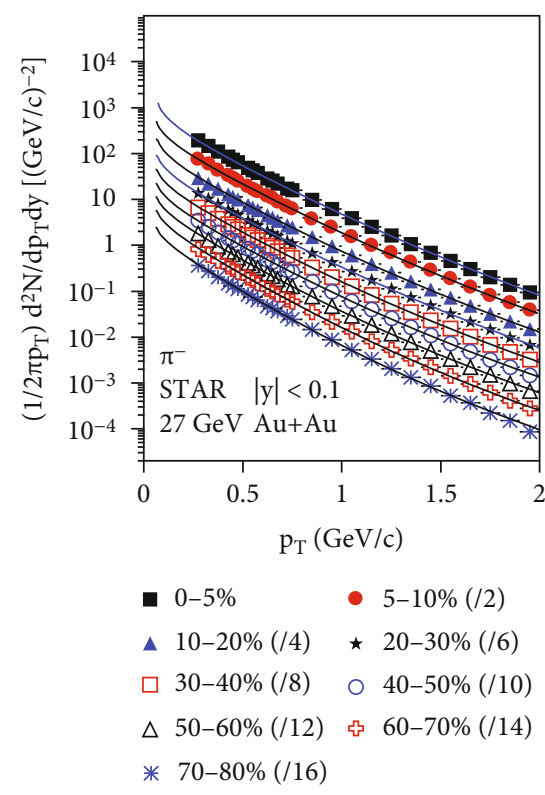

(a)

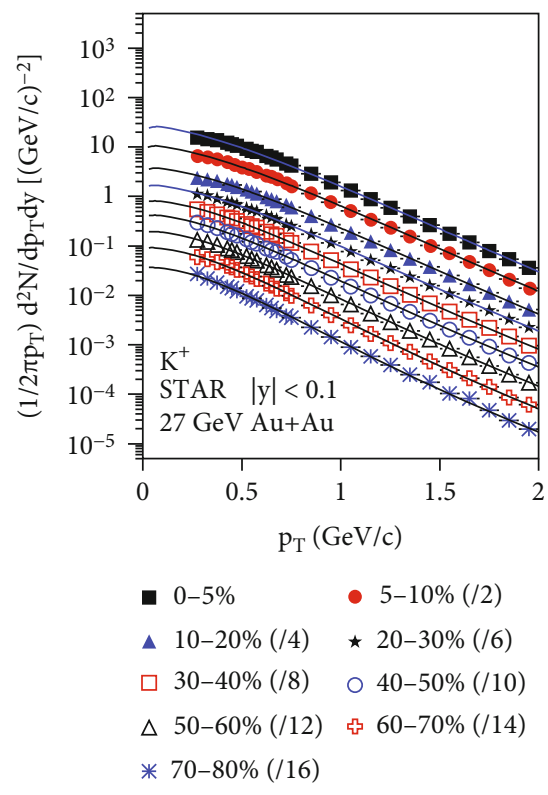

(d)

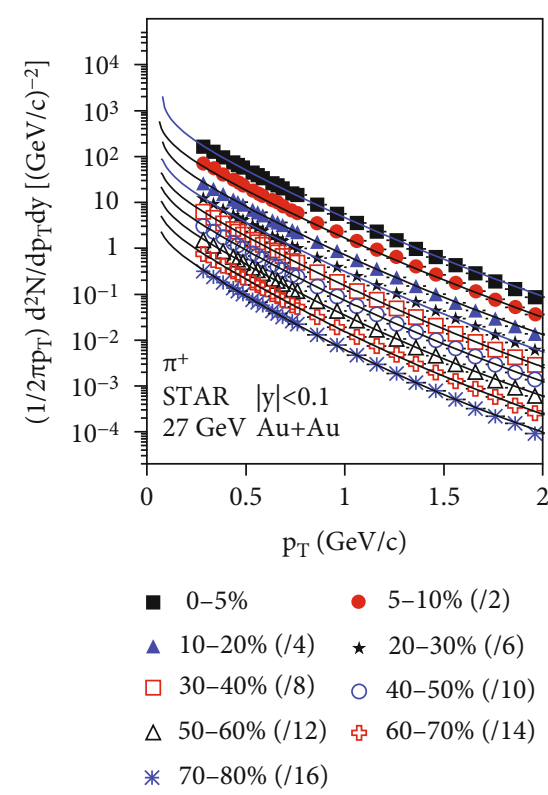

(b)

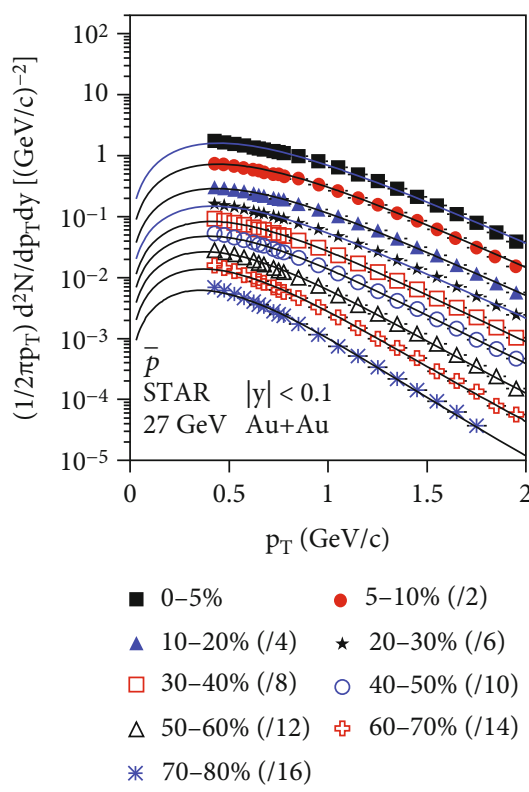

(e)

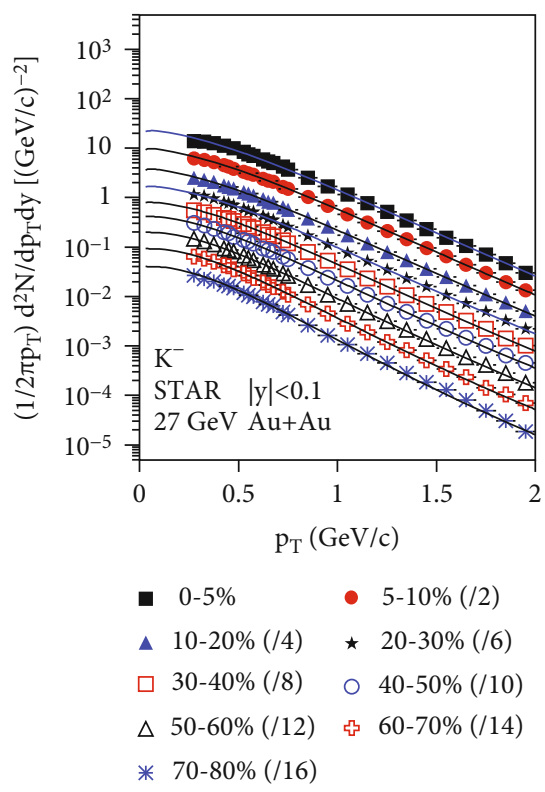

(c)

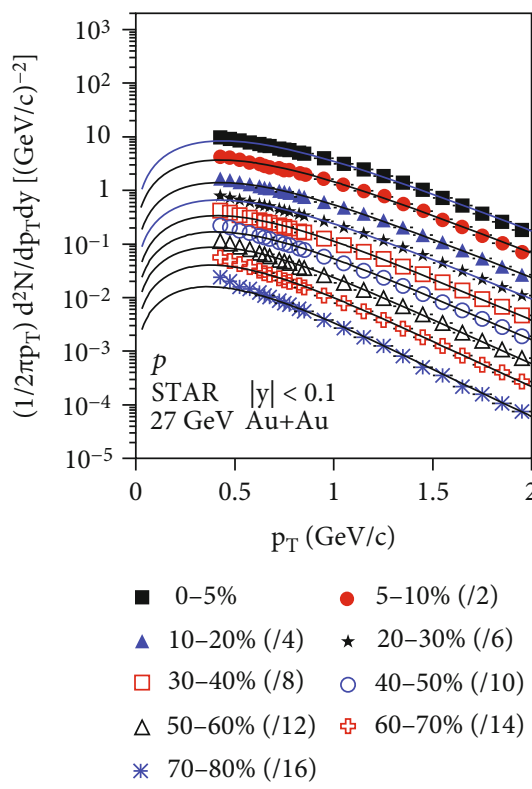

(f)

Figure 5: Same as Figure 1, but showing the results for $27 \mathrm{GeV}$ Au-Au collisions. The symbols represent the STAR data [33].

In some cases, the spectra in experiments are for $m_{T}$. We need to convert the probability density function $\left[f\left(p_{T}\right)\right]$ of $p_{T}$ to that $\left[f_{m_{T}}\left(m_{T}\right)\right]$ of $m_{T}$. In fact, we have the relation $f$ $\left(p_{T}\right)\left|d p_{T}\right|=f_{m_{T}}\left(m_{T}\right)\left|d m_{T}\right|$. Further, we have

$$
f_{m_{T}}\left(m_{T}\right)=\frac{m_{T}}{\sqrt{m_{T}^{2}-m_{0}^{2}}} f\left(\sqrt{m_{T}^{2}-m_{0}^{2}}\right) .
$$

For invariant $p_{T}$ and $m_{T}$ spectra which are used usually in experiments, we have

$$
\frac{1}{2 \pi p_{T}} \frac{d^{2} N}{d p_{T} d y}=\frac{1}{2 \pi m_{T}} \frac{d^{2} N}{d m_{T} d y} .
$$

Other forms of experimental spectra can be transformed from Equation (8) conveniently if $d y$ and the cross section are considered reasonably.

According to statistical physics and information theory, the essence of entropy is an expression of the degree of chaos existed inherently in a system $[51-53,71]$. Entropy has a certain relationship with the probability density function in thermodynamics. In order to further study the role of color confinement in relativistic heavy-ion collisions, the quantum 


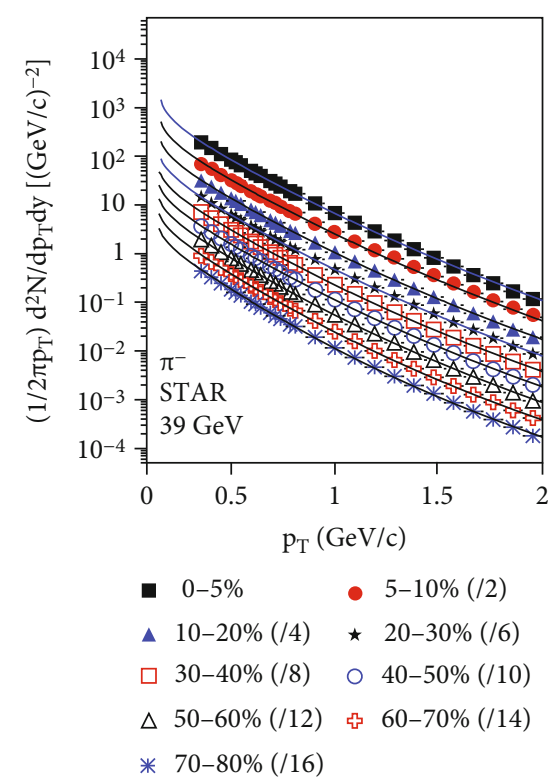

(a)

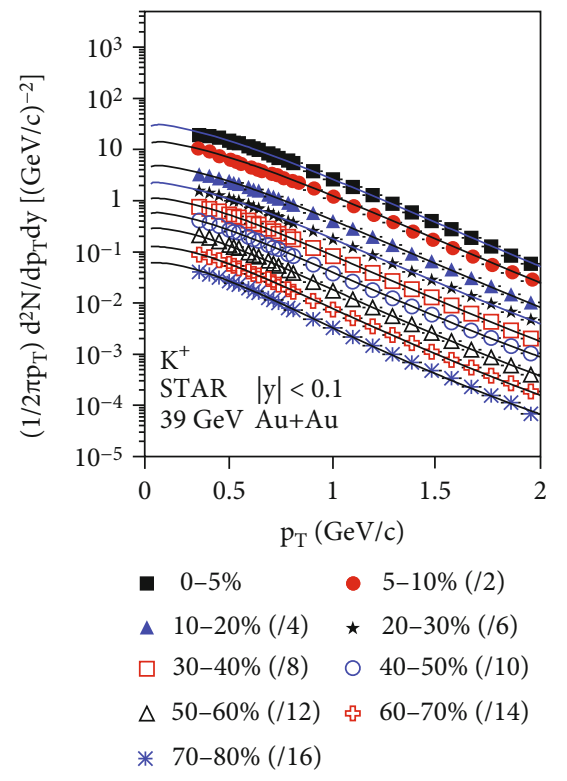

(d)

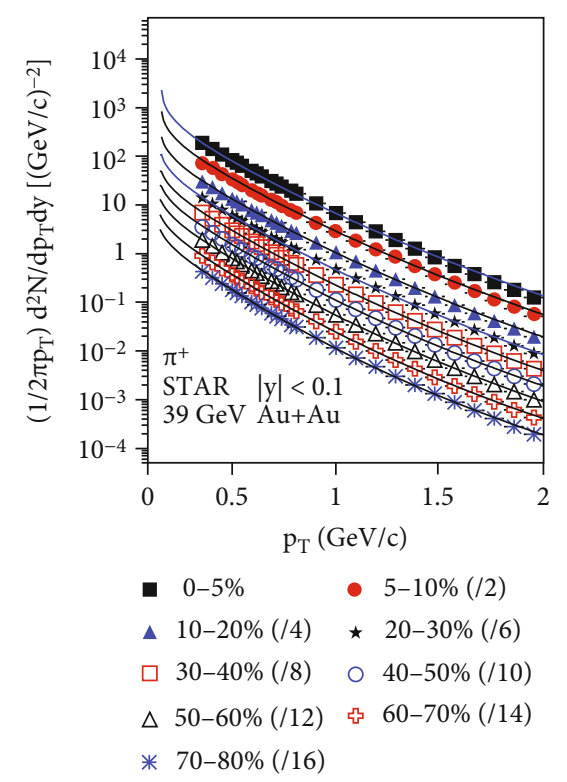

(b)

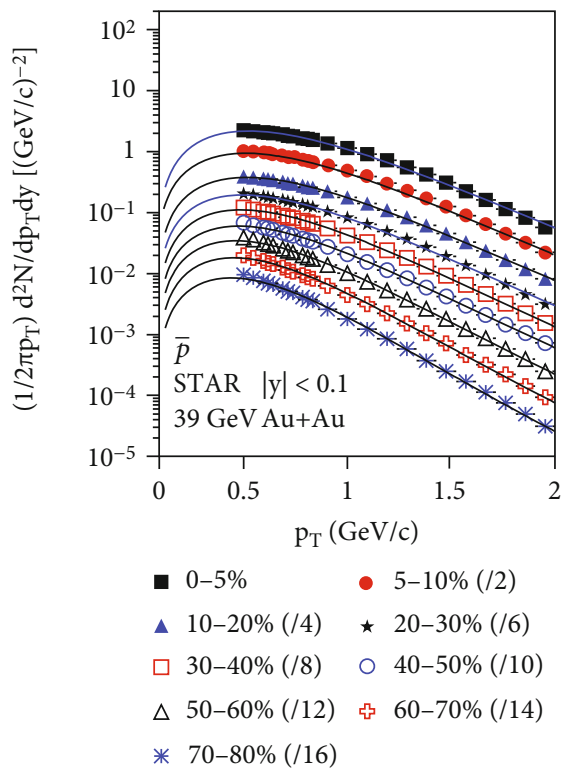

(e)

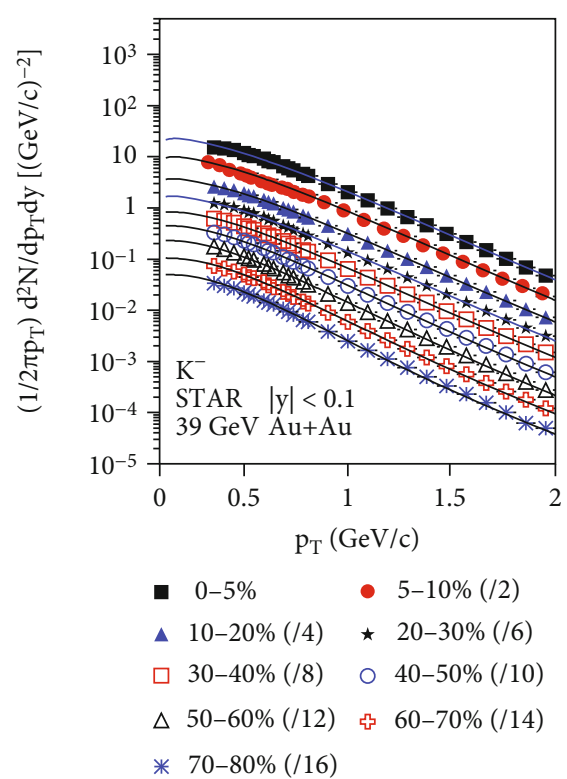

(c)

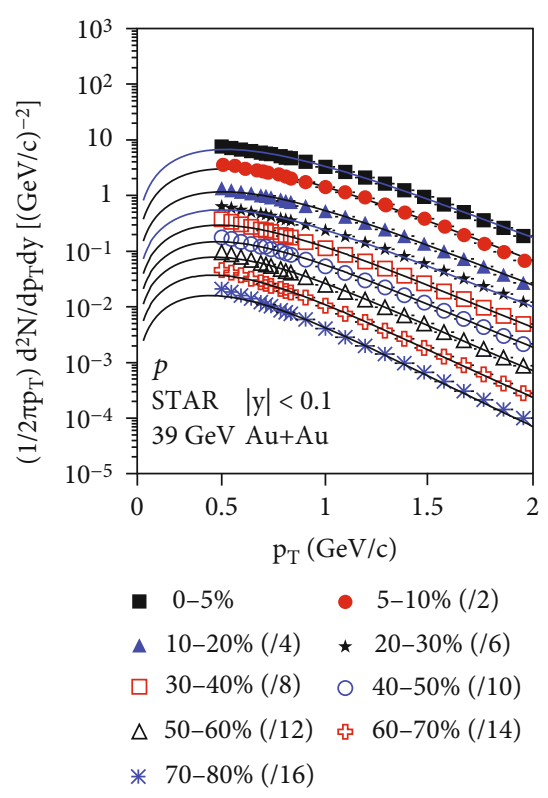

(f)

FIgURE 6: Same as Figure 1, but showing the results for $39 \mathrm{GeV} \mathrm{Au-Au} \mathrm{collisions.} \mathrm{The} \mathrm{symbols} \mathrm{represent} \mathrm{the} \mathrm{STAR} \mathrm{data} \mathrm{[33].}$

entanglement entropy (the entropy of gluons or partons) is proposed to explore the underlying mechanism. As we know, the relationship between the measured particle multiplicity distribution in the deep inelastic scattering process and the entropy of final hadrons is expressed as $[51-53,71]$

$$
S_{\text {hadron }}=-\sum P(N) \ln P(N) \text {, }
$$

where $P(N)$ is the multiplicity distribution of the charged particles measured in a given measurement area. The entropy of hadrons is assumed to be equal to the partonic entropy $[51-53,71]$.
The TP-like function and the corresponding convolutions, which are used to describe the $p_{t i}$ and $p_{T}$ distributions respectively, are also the probability density functions. Analogous to the multiplicity distribution of the above formula, for the first time, we define the pseudoentropy as follows:

$$
S_{\text {hadron }}^{\prime}=-\sum f\left(p_{T}\right) \ln f\left(p_{T}\right)
$$

in which the $p_{T}$ distribution $f\left(p_{T}\right)$ replaced the multiplicity distribution $P(N)$ in Equation (9). Different from $P(N)$ in which $N$ is discrete, $p_{T}$ in $f\left(p_{T}\right)$ is continuous. To give a suitable and uniform description, we set the bin width of $p_{T}$ to be $0.1 \mathrm{GeV} / c$ in the considered energy range in this paper. 
TABLE 2: Values of $n, T_{0}, a_{0},\left\langle\beta_{t}\right\rangle, \chi^{2}$, and ndof corresponding to the curves in Figure 2.

\begin{tabular}{|c|c|c|c|c|c|c|c|c|}
\hline Figure & Particle & $\sqrt{s_{N N}}(\mathrm{GeV})$ & Selection & $n$ & $T_{0}(\mathrm{GeV})$ & $a_{0}$ & $\left\langle\beta_{t}\right\rangle(c)$ & $\chi^{2} /$ ndof \\
\hline \multirow[t]{9}{*}{ Figure 2(a) } & $\pi^{-}$ & 11.5 & $0-5 \%$ & $16.0 \pm 0.6$ & $0.168 \pm 0.003$ & $-0.397 \pm 0.005$ & $0.114 \pm 0.003$ & $3 / 21$ \\
\hline & & $|y|<0.1$ & $5-10 \%$ & $14.5 \pm 0.5$ & $0.162 \pm 0.003$ & $-0.396 \pm 0.005$ & $0.113 \pm 0.003$ & $6 / 21$ \\
\hline & & & $10-20 \%$ & $13.4 \pm 0.4$ & $0.158 \pm 0.003$ & $-0.389 \pm 0.005$ & $0.110 \pm 0.003$ & $6 / 21$ \\
\hline & & & $20-30 \%$ & $13.4 \pm 0.4$ & $0.153 \pm 0.003$ & $-0.382 \pm 0.005$ & $0.109 \pm 0.003$ & $20 / 21$ \\
\hline & & & $30-40 \%$ & $13.2 \pm 0.4$ & $0.150 \pm 0.003$ & $-0.381 \pm 0.005$ & $0.107 \pm 0.003$ & $11 / 21$ \\
\hline & & & $40-50 \%$ & $13.3 \pm 0.4$ & $0.147 \pm 0.002$ & $-0.376 \pm 0.005$ & $0.103 \pm 0.003$ & $15 / 21$ \\
\hline & & & $50-60 \%$ & $12.5 \pm 0.3$ & $0.138 \pm 0.002$ & $-0.368 \pm 0.005$ & $0.100 \pm 0.002$ & $28 / 21$ \\
\hline & & & $60-70 \%$ & $12.3 \pm 0.3$ & $0.133 \pm 0.002$ & $-0.370 \pm 0.005$ & $0.099 \pm 0.002$ & $28 / 19$ \\
\hline & & & $70-80 \%$ & $12.0 \pm 0.3$ & $0.128 \pm 0.002$ & $-0.365 \pm 0.004$ & $0.094 \pm 0.002$ & $29 / 19$ \\
\hline \multirow[t]{9}{*}{ Figure 2(b) } & $\pi^{+}$ & 11.5 & $0-5 \%$ & $16.1 \pm 0.7$ & $0.175 \pm 0.003$ & $-0.422 \pm 0.005$ & $0.122 \pm 0.003$ & $3 / 21$ \\
\hline & & $|y|<0.1$ & $5-10 \%$ & $14.5 \pm 0.5$ & $0.170 \pm 0.003$ & $-0.420 \pm 0.005$ & $0.119 \pm 0.003$ & $5 / 21$ \\
\hline & & & $10-20 \%$ & $14.0 \pm 0.4$ & $0.166 \pm 0.003$ & $-0.417 \pm 0.005$ & $0.116 \pm 0.003$ & $5 / 21$ \\
\hline & & & $20-30 \%$ & $12.7 \pm 0.3$ & $0.161 \pm 0.003$ & $-0.407 \pm 0.005$ & $0.115 \pm 0.003$ & $4 / 21$ \\
\hline & & & $30-40 \%$ & $12.2 \pm 0.3$ & $0.156 \pm 0.003$ & $-0.400 \pm 0.005$ & $0.113 \pm 0.003$ & $3 / 21$ \\
\hline & & & $40-50 \%$ & $11.3 \pm 0.3$ & $0.149 \pm 0.002$ & $-0.391 \pm 0.005$ & $0.112 \pm 0.003$ & $6 / 21$ \\
\hline & & & $50-60 \%$ & $11.1 \pm 0.3$ & $0.140 \pm 0.002$ & $-0.386 \pm 0.005$ & $0.108 \pm 0.003$ & $10 / 21$ \\
\hline & & & $60-70 \%$ & $10.8 \pm 0.3$ & $0.135 \pm 0.002$ & $-0.378 \pm 0.005$ & $0.106 \pm 0.003$ & $8 / 19$ \\
\hline & & & $70-80 \%$ & $10.2 \pm 0.3$ & $0.129 \pm 0.002$ & $-0.374 \pm 0.005$ & $0.102 \pm 0.003$ & $20 / 19$ \\
\hline \multirow[t]{9}{*}{ Figure 2(c) } & $K^{-}$ & 11.5 & $0-5 \%$ & $26.9 \pm 1.6$ & $0.153 \pm 0.003$ & $0.007 \pm 0.004$ & $0.114 \pm 0.005$ & $1 / 18$ \\
\hline & & $|y|<0.1$ & $5-10 \%$ & $24.3 \pm 1.4$ & $0.152 \pm 0.003$ & $0.011 \pm 0.004$ & $0.111 \pm 0.005$ & $5 / 19$ \\
\hline & & & $10-20 \%$ & $22.9 \pm 1.2$ & $0.149 \pm 0.002$ & $0.012 \pm 0.004$ & $0.109 \pm 0.005$ & $4 / 19$ \\
\hline & & & $20-30 \%$ & $21.8 \pm 1.1$ & $0.145 \pm 0.002$ & $0.015 \pm 0.004$ & $0.107 \pm 0.005$ & $4 / 19$ \\
\hline & & & $30-40 \%$ & $20.9 \pm 1.0$ & $0.141 \pm 0.002$ & $0.022 \pm 0.004$ & $0.107 \pm 0.005$ & $4 / 18$ \\
\hline & & & $40-50 \%$ & $17.5 \pm 0.7$ & $0.128 \pm 0.002$ & $0.032 \pm 0.005$ & $0.106 \pm 0.005$ & $11 / 18$ \\
\hline & & & $50-60 \%$ & $15.6 \pm 0.6$ & $0.121 \pm 0.002$ & $0.051 \pm 0.005$ & $0.103 \pm 0.005$ & $12 / 18$ \\
\hline & & & $60-70 \%$ & $15.1 \pm 0.6$ & $0.110 \pm 0.002$ & $0.064 \pm 0.005$ & $0.102 \pm 0.005$ & $8 / 15$ \\
\hline & & & $70-80 \%$ & $15.0 \pm 0.5$ & $0.103 \pm 0.002$ & $0.076 \pm 0.005$ & $0.092 \pm 0.004$ & $18 / 11$ \\
\hline \multirow[t]{9}{*}{ Figure 2(d) } & $K^{+}$ & 11.5 & $0-5 \%$ & $28.3 \pm 1.8$ & $0.167 \pm 0.003$ & $-0.026 \pm 0.004$ & $0.127 \pm 0.005$ & $2 / 20$ \\
\hline & & $|y|<0.1$ & $5-10 \%$ & $27.4 \pm 1.7$ & $0.162 \pm 0.003$ & $-0.022 \pm 0.004$ & $0.121 \pm 0.005$ & $6 / 21$ \\
\hline & & & $10-20 \%$ & $24.9 \pm 1.4$ & $0.158 \pm 0.003$ & $-0.009 \pm 0.004$ & $0.115 \pm 0.005$ & $4 / 21$ \\
\hline & & & $20-30 \%$ & $23.1 \pm 1.3$ & $0.154 \pm 0.003$ & $0.003 \pm 0.004$ & $0.111 \pm 0.005$ & $8 / 21$ \\
\hline & & & $30-40 \%$ & $18.4 \pm 0.8$ & $0.149 \pm 0.002$ & $0.014 \pm 0.004$ & $0.108 \pm 0.005$ & $7 / 21$ \\
\hline & & & $40-50 \%$ & $17.0 \pm 0.7$ & $0.141 \pm 0.002$ & $0.019 \pm 0.004$ & $0.101 \pm 0.005$ & $16 / 21$ \\
\hline & & & $50-60 \%$ & $14.5 \pm 0.5$ & $0.134 \pm 0.002$ & $0.022 \pm 0.004$ & $0.099 \pm 0.004$ & $12 / 20$ \\
\hline & & & $60-70 \%$ & $13.9 \pm 0.4$ & $0.126 \pm 0.002$ & $0.032 \pm 0.005$ & $0.091 \pm 0.004$ & $5 / 18$ \\
\hline & & & $70-80 \%$ & $13.9 \pm 0.4$ & $0.116 \pm 0.002$ & $0.041 \pm 0.005$ & $0.089 \pm 0.004$ & $13 / 17$ \\
\hline
\end{tabular}


TABLE 2: Continued.

\begin{tabular}{|c|c|c|c|c|c|c|c|c|}
\hline Figure & Particle & $\sqrt{s_{N N}}(\mathrm{GeV})$ & Selection & $n$ & $T_{0}(\mathrm{GeV})$ & $a_{0}$ & $\left\langle\beta_{t}\right\rangle(c)$ & $\chi^{2} /$ ndof \\
\hline \multirow[t]{9}{*}{ Figure 2(e) } & $\bar{p}$ & 11.5 & $0-5 \%$ & $16.1 \pm 0.7$ & $0.128 \pm 0.002$ & $0.003 \pm 0.004$ & $0.346 \pm 0.008$ & $19 / 18$ \\
\hline & & $|y|<0.1$ & $5-10 \%$ & $15.6 \pm 0.6$ & $0.124 \pm 0.002$ & $0.006 \pm 0.004$ & $0.330 \pm 0.008$ & $12 / 18$ \\
\hline & & & $10-20 \%$ & $15.1 \pm 0.6$ & $0.118 \pm 0.002$ & $0.010 \pm 0.004$ & $0.326 \pm 0.008$ & $13 / 18$ \\
\hline & & & $20-30 \%$ & $14.7 \pm 0.5$ & $0.116 \pm 0.002$ & $0.012 \pm 0.004$ & $0.314 \pm 0.008$ & $5 / 18$ \\
\hline & & & $30-40 \%$ & $14.0 \pm 0.4$ & $0.107 \pm 0.002$ & $0.015 \pm 0.004$ & $0.306 \pm 0.008$ & $3 / 18$ \\
\hline & & & $40-50 \%$ & $13.2 \pm 0.4$ & $0.098 \pm 0.002$ & $0.017 \pm 0.004$ & $0.296 \pm 0.007$ & $8 / 15$ \\
\hline & & & $50-60 \%$ & $12.1 \pm 0.3$ & $0.091 \pm 0.002$ & $0.020 \pm 0.004$ & $0.292 \pm 0.007$ & $7 / 14$ \\
\hline & & & $60-70 \%$ & $11.1 \pm 0.3$ & $0.081 \pm 0.001$ & $0.021 \pm 0.004$ & $0.284 \pm 0.007$ & $7 / 9$ \\
\hline & & & $70-80 \%$ & $10.1 \pm 0.3$ & $0.072 \pm 0.001$ & $0.022 \pm 0.004$ & $0.272 \pm 0.007$ & $6 / 9$ \\
\hline \multirow[t]{9}{*}{ Figure 2(f) } & $p$ & 11.5 & $0-5 \%$ & $17.8 \pm 0.7$ & $0.129 \pm 0.002$ & $0.001 \pm 0.004$ & $0.347 \pm 0.008$ & $8 / 23$ \\
\hline & & $|y|<0.1$ & $5-10 \%$ & $16.2 \pm 0.7$ & $0.128 \pm 0.002$ & $0.004 \pm 0.004$ & $0.333 \pm 0.008$ & $2 / 24$ \\
\hline & & & $10-20 \%$ & $14.9 \pm 0.5$ & $0.122 \pm 0.002$ & $0.011 \pm 0.004$ & $0.327 \pm 0.008$ & $5 / 24$ \\
\hline & & & $20-30 \%$ & $13.8 \pm 0.4$ & $0.119 \pm 0.002$ & $0.013 \pm 0.004$ & $0.314 \pm 0.008$ & $7 / 24$ \\
\hline & & & $30-40 \%$ & $13.1 \pm 0.4$ & $0.115 \pm 0.002$ & $0.015 \pm 0.004$ & $0.302 \pm 0.008$ & $12 / 24$ \\
\hline & & & $40-50 \%$ & $12.4 \pm 0.3$ & $0.108 \pm 0.002$ & $0.017 \pm 0.004$ & $0.290 \pm 0.007$ & $11 / 23$ \\
\hline & & & $50-60 \%$ & $11.7 \pm 0.3$ & $0.104 \pm 0.002$ & $0.018 \pm 0.004$ & $0.274 \pm 0.007$ & $14 / 23$ \\
\hline & & & $60-70 \%$ & $11.1 \pm 0.3$ & $0.094 \pm 0.002$ & $0.021 \pm 0.004$ & $0.269 \pm 0.007$ & $18 / 23$ \\
\hline & & & $70-80 \%$ & $10.8 \pm 0.3$ & $0.088 \pm 0.001$ & $0.023 \pm 0.004$ & $0.265 \pm 0.007$ & $30 / 24$ \\
\hline
\end{tabular}

Although the pseudoentropy is the bin width dependent, we may compare the relative sizes in different cases if the bin width is beforehand fixed.

\section{Results and Discussion}

3.1. Comparison with Data. Figure 1 shows the $p_{T}$ spectra, $\left(1 / 2 \pi p_{T}\right) d^{2} N / d p_{T} d y$, of $\pi^{-}$(a), $\pi^{+}(\mathrm{b}), K^{-}$(c), $K^{+}(\mathrm{d}), \bar{p}$ (e), and $p$ (f) produced at mid- $y(|y|<0.1)$ in Au-Au collisions at center-of-mass energy $\sqrt{s_{N N}}=7.7 \mathrm{GeV}$ (per nucleon pair) with different centrality intervals. The symbols represent the experimental data measured by the STAR Collaboration in the RHIC-BES project $[33,34]$ and rescaled by different amounts marked in the panels for clarity. The curves in Figures 1(a)-1(d) represent the results fitted by the convolution of two TP-like functions, for the spectra of $\pi^{\mp}$ and $K^{\mp}$. The curves for the spectra of $\bar{p}(\mathrm{e})$ and $p(\mathrm{f})$ are the results fitted by the convolution of three TP-like functions. We have used the method of least squares to determine the values of parameters and the method of statistical simulation to determine the errors of parameters. The values of $n, T_{0}, a_{0},\left\langle\beta_{t}\right\rangle, \chi^{2}$, and degrees of freedom (ndof) are listed in Table 1. It can be seen that the convolution of two (three) TP-like functions fits well the experimental spectra of $\pi^{\mp}$ and $K^{\mp}(\bar{p}$ and $p$ ) produced in $7.7 \mathrm{GeV} \mathrm{Au}$-Au collisions with different centrality intervals in the available $p_{T}$ range.

Similarly, the $p_{T}$ spectra of $\pi^{-}(\mathrm{a}), \pi^{+}(\mathrm{b}), K^{-}(\mathrm{c}), K^{+}(\mathrm{d})$, $\bar{p}(\mathrm{e})$, and $p(\mathrm{f})$ produced at mid- $y$ in Au-Au collisions with different centrality intervals at $\sqrt{s_{N N}}=11.5,14.5,19.6,27$, and $39 \mathrm{GeV}$ are demonstrated in Figures 2-6, respectively, where the data are cited from the STAR Collaboration [33,
34]. The values of parameters, $\chi^{2}$, and ndof are listed in Tables 2-6 for different energies. One can see that the convolution of two (three) TP-like functions fits well the experimental spectra of $\pi^{\mp}$ and $K^{\mp}$ ( $\bar{p}$ and $p$ ) produced in $\mathrm{Au}-\mathrm{Au}$ collisions at $11.5,14.5,19.6,27$, and $39 \mathrm{GeV}$ with different centrality intervals in the available $p_{T}$ range.

In order to better explore the change trends of parameters, besides the RHIC-BES energies, we have also conducted data collection and research on the $p_{T}$ spectra at other RHIC energies. Figures 7-9 show the $p_{T}$ spectra of $\pi^{-}(a), \pi^{+}(b)$, $K^{-}(\mathrm{c}), K^{+}(\mathrm{d}), \bar{p}(\mathrm{e})$, and $p$ (f) produced in Au-Au collisions at $62.4,130$, and $200 \mathrm{GeV}$, respectively. The data with $|y|<$ 0.1 are also cited from the STAR Collaboration [35]. The centrality intervals at 62.4 and $200 \mathrm{GeV}$ are the same as those at RHIC-BES energies. However, the centrality intervals at $130 \mathrm{GeV}$ are somehow different from others. The related parameters are listed in Tables 7-9 for different energies. One can see again that the convolution of two (three) TPlike functions fits well the experimental spectra of $\pi^{\mp}$ and $K^{\mp}(\bar{p}$ and $p)$ produced in Au-Au collisions at 62.4, 130, and $200 \mathrm{GeV}$ with different centrality intervals in the available $p_{T}$ range.

Except the above comparisons, we have studied the $m_{T}$ spectra of $\pi^{-}(\mathrm{a}), \pi^{+}(\mathrm{b}), K^{-}(\mathrm{c}), K^{+}(\mathrm{d})$, and $p$ (e) produced at mid- $y$ in $0-5 \%$ Au-Au collisions at $\sqrt{s_{N N}}=2.70,3.32$, $3.84,4.30$, and $4.88 \mathrm{GeV}$ in Figure 10, where the five energies are only available in panel (d). The symbols for $\pi^{\mp}$ in $|y|<$ $0.05, K^{\mp}$ in $|y|<0.25$, and $p$ in $|y|<0.05$ represent the experimental data measured by the E895 [36], E866 [37], and E895 [38] Collaborations, respectively, where some sets of data are rescaled by different amounts for clarity. The data 
TABLE 3: Values of $n, T_{0}, a_{0},\left\langle\beta_{t}\right\rangle, \chi^{2}$, and ndof corresponding to the curves in Figure 3.

\begin{tabular}{|c|c|c|c|c|c|c|c|c|}
\hline Figure & Particle & $\sqrt{s_{N N}}(\mathrm{GeV})$ & Selection & $n$ & $T_{0}(\mathrm{GeV})$ & $a_{0}$ & $\left\langle\beta_{t}\right\rangle(c)$ & $\chi^{2} /$ ndof \\
\hline \multirow[t]{9}{*}{ Figure 3(a) } & $\pi^{-}$ & 14.5 & $0-5 \%$ & $14.3 \pm 0.5$ & $0.171 \pm 0.003$ & $-0.407 \pm 0.005$ & $0.117 \pm 0.003$ & $1 / 23$ \\
\hline & & $|y|<0.1$ & $5-10 \%$ & $12.6 \pm 0.3$ & $0.165 \pm 0.003$ & $-0.405 \pm 0.005$ & $0.114 \pm 0.003$ & $2 / 23$ \\
\hline & & & $10-20 \%$ & $11.9 \pm 0.3$ & $0.161 \pm 0.003$ & $-0.396 \pm 0.005$ & $0.112 \pm 0.003$ & $3 / 23$ \\
\hline & & & $20-30 \%$ & $11.5 \pm 0.3$ & $0.158 \pm 0.003$ & $-0.392 \pm 0.005$ & $0.110 \pm 0.003$ & $4 / 23$ \\
\hline & & & $30-40 \%$ & $11.0 \pm 0.3$ & $0.153 \pm 0.003$ & $-0.385 \pm 0.005$ & $0.108 \pm 0.003$ & $4 / 23$ \\
\hline & & & $40-50 \%$ & $10.8 \pm 0.3$ & $0.150 \pm 0.003$ & $-0.378 \pm 0.005$ & $0.104 \pm 0.003$ & $3 / 23$ \\
\hline & & & $50-60 \%$ & $10.7 \pm 0.3$ & $0.141 \pm 0.002$ & $-0.377 \pm 0.005$ & $0.102 \pm 0.003$ & $13 / 23$ \\
\hline & & & $60-70 \%$ & $10.4 \pm 0.3$ & $0.133 \pm 0.002$ & $-0.370 \pm 0.005$ & $0.101 \pm 0.003$ & $25 / 23$ \\
\hline & & & $70-80 \%$ & $10.3 \pm 0.3$ & $0.128 \pm 0.002$ & $-0.368 \pm 0.004$ & $0.098 \pm 0.002$ & $25 / 23$ \\
\hline \multirow[t]{9}{*}{ Figure 3(b) } & $\pi^{+}$ & 14.5 & $0-5 \%$ & $14.6 \pm 0.5$ & $0.179 \pm 0.003$ & $-0.424 \pm 0.005$ & $0.124 \pm 0.003$ & $5 / 23$ \\
\hline & & $|y|<0.1$ & $5-10 \%$ & $12.7 \pm 0.3$ & $0.172 \pm 0.003$ & $-0.421 \pm 0.005$ & $0.122 \pm 0.003$ & $2 / 23$ \\
\hline & & & $10-20 \%$ & $11.9 \pm 0.3$ & $0.167 \pm 0.003$ & $-0.419 \pm 0.005$ & $0.120 \pm 0.003$ & $4 / 23$ \\
\hline & & & $20-30 \%$ & $11.4 \pm 0.3$ & $0.163 \pm 0.003$ & $-0.416 \pm 0.005$ & $0.116 \pm 0.003$ & $4 / 23$ \\
\hline & & & $30-40 \%$ & $10.9 \pm 0.3$ & $0.159 \pm 0.003$ & $-0.411 \pm 0.005$ & $0.114 \pm 0.003$ & $3 / 23$ \\
\hline & & & $40-50 \%$ & $10.4 \pm 0.3$ & $0.151 \pm 0.003$ & $-0.404 \pm 0.005$ & $0.112 \pm 0.003$ & $1 / 23$ \\
\hline & & & $50-60 \%$ & $10.2 \pm 0.3$ & $0.143 \pm 0.002$ & $-0.396 \pm 0.005$ & $0.110 \pm 0.003$ & $7 / 23$ \\
\hline & & & $60-70 \%$ & $10.0 \pm 0.3$ & $0.137 \pm 0.002$ & $-0.394 \pm 0.005$ & $0.108 \pm 0.003$ & $14 / 23$ \\
\hline & & & $70-80 \%$ & $9.9 \pm 0.2$ & $0.129 \pm 0.002$ & $-0.384 \pm 0.005$ & $0.105 \pm 0.003$ & $26 / 23$ \\
\hline \multirow[t]{9}{*}{ Figure 3(c) } & $K^{-}$ & 14.5 & $0-5 \%$ & $23.3 \pm 1.3$ & $0.159 \pm 0.003$ & $-0.015 \pm 0.004$ & $0.116 \pm 0.005$ & $2 / 21$ \\
\hline & & $|y|<0.1$ & $5-10 \%$ & $20.8 \pm 1.0$ & $0.157 \pm 0.003$ & $-0.013 \pm 0.004$ & $0.113 \pm 0.005$ & $2 / 21$ \\
\hline & & & $10-20 \%$ & $20.3 \pm 1.0$ & $0.152 \pm 0.003$ & $0.002 \pm 0.004$ & $0.112 \pm 0.005$ & $2 / 21$ \\
\hline & & & $20-30 \%$ & $18.9 \pm 0.8$ & $0.148 \pm 0.002$ & $0.014 \pm 0.004$ & $0.113 \pm 0.005$ & $7 / 21$ \\
\hline & & & $30-40 \%$ & $18.4 \pm 0.8$ & $0.143 \pm 0.002$ & $0.026 \pm 0.004$ & $0.107 \pm 0.005$ & $6 / 21$ \\
\hline & & & $40-50 \%$ & $13.7 \pm 0.4$ & $0.130 \pm 0.002$ & $0.029 \pm 0.004$ & $0.105 \pm 0.005$ & $5 / 19$ \\
\hline & & & $50-60 \%$ & $14.0 \pm 0.4$ & $0.122 \pm 0.002$ & $0.044 \pm 0.005$ & $0.102 \pm 0.005$ & $6 / 17$ \\
\hline & & & $60-70 \%$ & $13.2 \pm 0.3$ & $0.109 \pm 0.002$ & $0.053 \pm 0.005$ & $0.101 \pm 0.005$ & $6 / 16$ \\
\hline & & & $70-80 \%$ & $12.1 \pm 0.3$ & $0.104 \pm 0.002$ & $0.062 \pm 0.005$ & $0.098 \pm 0.004$ & $3 / 15$ \\
\hline \multirow[t]{9}{*}{ Figure $3(\mathrm{~d})$} & $K^{+}$ & 14.5 & $0-5 \%$ & $26.6 \pm 1.6$ & $0.170 \pm 0.003$ & $-0.037 \pm 0.005$ & $0.129 \pm 0.005$ & $0.9 / 21$ \\
\hline & & $|y|<0.1$ & $5-10 \%$ & $22.1 \pm 1.2$ & $0.163 \pm 0.003$ & $-0.034 \pm 0.005$ & $0.125 \pm 0.005$ & $3 / 21$ \\
\hline & & & $10-20 \%$ & $17.8 \pm 0.7$ & $0.162 \pm 0.003$ & $-0.024 \pm 0.004$ & $0.123 \pm 0.005$ & $1 / 21$ \\
\hline & & & $20-30 \%$ & $16.7 \pm 0.7$ & $0.158 \pm 0.003$ & $-0.020 \pm 0.004$ & $0.112 \pm 0.005$ & $2 / 21$ \\
\hline & & & $30-40 \%$ & $15.9 \pm 0.6$ & $0.151 \pm 0.003$ & $-0.017 \pm 0.004$ & $0.108 \pm 0.005$ & $5 / 21$ \\
\hline & & & $40-50 \%$ & $13.6 \pm 0.4$ & $0.142 \pm 0.002$ & $-0.008 \pm 0.004$ & $0.105 \pm 0.005$ & $10 / 19$ \\
\hline & & & $50-60 \%$ & $11.8 \pm 0.3$ & $0.137 \pm 0.002$ & $0.012 \pm 0.004$ & $0.101 \pm 0.005$ & $6 / 19$ \\
\hline & & & $60-70 \%$ & $11.3 \pm 0.3$ & $0.127 \pm 0.002$ & $0.015 \pm 0.004$ & $0.095 \pm 0.004$ & $4 / 17$ \\
\hline & & & $70-80 \%$ & $12.3 \pm 0.3$ & $0.115 \pm 0.002$ & $0.025 \pm 0.004$ & $0.091 \pm 0.004$ & $2 / 15$ \\
\hline
\end{tabular}


TABLE 3: Continued.

\begin{tabular}{|c|c|c|c|c|c|c|c|c|}
\hline Figure & Particle & $\sqrt{s_{N N}}(\mathrm{GeV})$ & Selection & $n$ & $T_{0}(\mathrm{GeV})$ & $a_{0}$ & $\left\langle\beta_{t}\right\rangle(c)$ & $\chi^{2} /$ ndof \\
\hline \multirow[t]{9}{*}{ Figure 3(e) } & $\bar{p}$ & 14.5 & $0-5 \%$ & $15.9 \pm 0.6$ & $0.127 \pm 0.002$ & $0.001 \pm 0.004$ & $0.358 \pm 0.008$ & $8 / 20$ \\
\hline & & $|y|<0.1$ & $5-10 \%$ & $14.6 \pm 0.5$ & $0.124 \pm 0.002$ & $0.004 \pm 0.004$ & $0.344 \pm 0.008$ & $9 / 20$ \\
\hline & & & $10-20 \%$ & $13.7 \pm 0.4$ & $0.120 \pm 0.002$ & $0.008 \pm 0.004$ & $0.339 \pm 0.008$ & $7 / 20$ \\
\hline & & & $20-30 \%$ & $13.3 \pm 0.4$ & $0.117 \pm 0.002$ & $0.011 \pm 0.004$ & $0.333 \pm 0.008$ & $5 / 20$ \\
\hline & & & $30-40 \%$ & $11.5 \pm 0.3$ & $0.108 \pm 0.002$ & $0.014 \pm 0.004$ & $0.324 \pm 0.008$ & $5 / 20$ \\
\hline & & & $40-50 \%$ & $11.7 \pm 0.3$ & $0.097 \pm 0.002$ & $0.016 \pm 0.004$ & $0.318 \pm 0.008$ & $2 / 18$ \\
\hline & & & $50-60 \%$ & $11.7 \pm 0.3$ & $0.089 \pm 0.001$ & $0.018 \pm 0.004$ & $0.312 \pm 0.008$ & $4 / 16$ \\
\hline & & & $60-70 \%$ & $10.5 \pm 0.3$ & $0.078 \pm 0.001$ & $0.020 \pm 0.004$ & $0.306 \pm 0.008$ & $3 / 11$ \\
\hline & & & $70-80 \%$ & $9.9 \pm 0.2$ & $0.073 \pm 0.001$ & $0.021 \pm 0.004$ & $0.298 \pm 0.007$ & $3 / 10$ \\
\hline \multirow[t]{9}{*}{ Figure 3(f) } & $p$ & 14.5 & $0-5 \%$ & $17.0 \pm 0.7$ & $0.133 \pm 0.002$ & $-0.001 \pm 0.004$ & $0.350 \pm 0.008$ & $1 / 20$ \\
\hline & & $|y|<0.1$ & $5-10 \%$ & $15.4 \pm 0.6$ & $0.131 \pm 0.002$ & $0.001 \pm 0.004$ & $0.343 \pm 0.008$ & $0.9 / 20$ \\
\hline & & & $10-20 \%$ & $13.8 \pm 0.4$ & $0.126 \pm 0.002$ & $0.008 \pm 0.004$ & $0.331 \pm 0.008$ & $0.9 / 20$ \\
\hline & & & $20-30 \%$ & $13.1 \pm 0.4$ & $0.122 \pm 0.002$ & $0.011 \pm 0.004$ & $0.318 \pm 0.008$ & $2 / 20$ \\
\hline & & & $30-40 \%$ & $12.6 \pm 0.3$ & $0.118 \pm 0.002$ & $0.014 \pm 0.004$ & $0.304 \pm 0.008$ & $3 / 20$ \\
\hline & & & $40-50 \%$ & $12.1 \pm 0.3$ & $0.116 \pm 0.002$ & $0.016 \pm 0.004$ & $0.298 \pm 0.007$ & $7 / 20$ \\
\hline & & & $50-60 \%$ & $11.5 \pm 0.3$ & $0.107 \pm 0.002$ & $0.017 \pm 0.004$ & $0.290 \pm 0.007$ & $7 / 20$ \\
\hline & & & $60-70 \%$ & $10.9 \pm 0.3$ & $0.103 \pm 0.002$ & $0.020 \pm 0.004$ & $0.283 \pm 0.007$ & $4 / 20$ \\
\hline & & & $70-80 \%$ & $10.6 \pm 0.3$ & $0.093 \pm 0.002$ & $0.021 \pm 0.004$ & $0.272 \pm 0.007$ & $19 / 20$ \\
\hline
\end{tabular}

for $\bar{p}$ is not available in the same or similar experiments due to low energy. The related parameters are listed in Table 10. One can see that the convolution of two (three) TP-like functions fits approximately the experimental spectra of $\pi^{\mp}$ and $K^{\mp}(p)$ produced in $0-5 \% \mathrm{Au}-\mathrm{Au}$ collisions at 2.70, $3.32,3.84,4.30$, and $4.88 \mathrm{GeV}$.

3.2. Tendencies of Parameters. We now analyze the tendencies of parameters on collision energy $\sqrt{s_{N N}}$ and centrality interval (percentage). In Figure 11, the dependence of parameter $n$ on $\sqrt{s_{N N}}$ and centrality is shown, where panels (a)-(f) are for the results from the spectra of $\pi^{-}, \pi^{+}, K^{-}, K^{+}, \bar{p}$, and $p$, respectively. It is not difficult to find out that with the increase of $\sqrt{s_{N N}}$, the values of $n$ decrease significantly. Meanwhile, with the decrease of centrality from central to peripheral collisions, the values of $n$ also decrease. As we know, $n$ is used to describe the degree of nonequilibrium of the system. The larger the value of $n$ is, the closer to equilibrium the system is. This work shows that the system at lower energy and in central collisions corresponds to larger $n$ and hence to higher degree of equilibrium, compared with the system at higher energy and in peripheral collisions.

As one of the core concepts in thermodynamics and statistical mechanics, temperature is also the attention of our research. Generally, we can directly extract the temperature information of the system from particle spectra. Panels (a)-(f) in Figure 12 show the dependence of thermal freeze-out temperature $T_{0}$ on $\sqrt{s_{N N}}$ extracted from the spectra of six kinds of charged hadrons in different centrality intervals. It can be clearly seen that in the available central collisions, $T_{0}$ increases rapidly in $\sqrt{s_{N N}}=2.7-7.7 \mathrm{GeV}$ range. When $\sqrt{s_{N N}}$ is larger than $7.7 \mathrm{GeV}, T_{0}$ increases slowly. The higher the temperature is, the higher the excitation degree is. As $\sqrt{s_{N N}}$ increases, the excitation degree of the system also increases. The change trend of $T_{0}$ with $\sqrt{s_{N N}}$ can well reflect this change phenomenon of the excitation degree. At nine collision energies and for six kinds of particles, $T_{0}$ decreases with the decrease of the centrality from central to peripheral collisions.

Similar to Figures 11 and 12, the dependence of the dimensionless correction index $a_{0}$ on $\sqrt{s_{N N}}$ is shown in Figures 13(a)-13(f) for different centrality intervals and charged hadrons. From Figure 13, one can see that with the increase of $\sqrt{s_{N N}}$, in the available central collisions, $a_{0}$ increases quickly and then decreases significantly, and the boundary energy is $7.7 \mathrm{GeV}$. The values of $a_{0}$ from $\pi^{\mp}$ spectra are negative, which is significantly less than those from $K^{\mp}$ and $\bar{p}(p)$ spectra. From central to peripheral collisions, $a_{0}$ increases significantly for different kinds of particles in the available energy range.

Figure 14 demonstrates the relationship between the average transverse flow velocity $\left\langle\beta_{t}\right\rangle$ and $\sqrt{s_{N N}}$ for different particles in panels (a)-(f), where the results for different centrality intervals are shown. With increasing $\sqrt{s_{N N}}$, one can see a significantly increasing $\left\langle\beta_{t}\right\rangle$ for different hadrons in most cases. At different energies, $\left\langle\beta_{t}\right\rangle$ for each kind of particles from central to peripheral collisions decreases gradually. Observing the values of $\left\langle\beta_{t}\right\rangle$, one can clearly find that the order of flow velocity is $\bar{p}(p)>K^{\mp}>\pi^{\mp}$. 
TABLE 4: Values of $n, T_{0}, a_{0},\left\langle\beta_{t}\right\rangle, \chi^{2}$, and ndof corresponding to the curves in Figure 4.

\begin{tabular}{|c|c|c|c|c|c|c|c|c|}
\hline Figure & Particle & $\sqrt{s_{N N}}(\mathrm{GeV})$ & Selection & $n$ & $T_{0}(\mathrm{GeV})$ & $a_{0}$ & $\left\langle\beta_{t}\right\rangle(c)$ & $\chi^{2} /$ ndof \\
\hline \multirow[t]{9}{*}{ Figure 4(a) } & $\pi^{-}$ & 19.6 & $0-5 \%$ & $12.9 \pm 0.3$ & $0.173 \pm 0.003$ & $-0.410 \pm 0.005$ & $0.119 \pm 0.003$ & $1 / 21$ \\
\hline & & $|y|<0.1$ & $5-10 \%$ & $11.5 \pm 0.3$ & $0.168 \pm 0.003$ & $-0.409 \pm 0.005$ & $0.117 \pm 0.003$ & $2 / 21$ \\
\hline & & & $10-20 \%$ & $10.9 \pm 0.3$ & $0.164 \pm 0.003$ & $-0.402 \pm 0.005$ & $0.115 \pm 0.003$ & $2 / 21$ \\
\hline & & & $20-30 \%$ & $10.3 \pm 0.3$ & $0.161 \pm 0.003$ & $-0.404 \pm 0.005$ & $0.112 \pm 0.003$ & $2 / 21$ \\
\hline & & & $30-40 \%$ & $10.0 \pm 0.3$ & $0.157 \pm 0.003$ & $-0.395 \pm 0.005$ & $0.109 \pm 0.003$ & $3 / 21$ \\
\hline & & & $40-50 \%$ & $9.8 \pm 0.2$ & $0.153 \pm 0.003$ & $-0.390 \pm 0.005$ & $0.107 \pm 0.003$ & $3 / 21$ \\
\hline & & & $50-60 \%$ & $9.4 \pm 0.2$ & $0.145 \pm 0.002$ & $-0.383 \pm 0.005$ & $0.104 \pm 0.003$ & $4 / 21$ \\
\hline & & & $60-70 \%$ & $9.0 \pm 0.2$ & $0.136 \pm 0.002$ & $-0.377 \pm 0.005$ & $0.103 \pm 0.003$ & $13 / 21$ \\
\hline & & & $70-80 \%$ & $8.9 \pm 0.2$ & $0.131 \pm 0.002$ & $-0.372 \pm 0.005$ & $0.102 \pm 0.003$ & $13 / 20$ \\
\hline \multirow[t]{9}{*}{ Figure 4(b) } & $\pi^{+}$ & 19.6 & $0-5 \%$ & $12.9 \pm 0.3$ & $0.183 \pm 0.003$ & $-0.424 \pm 0.005$ & $0.125 \pm 0.003$ & $14 / 21$ \\
\hline & & $|y|<0.1$ & $5-10 \%$ & $11.9 \pm 0.3$ & $0.177 \pm 0.003$ & $-0.420 \pm 0.005$ & $0.124 \pm 0.003$ & $6 / 21$ \\
\hline & & & $10-20 \%$ & $11.4 \pm 0.3$ & $0.170 \pm 0.003$ & $-0.417 \pm 0.005$ & $0.121 \pm 0.003$ & $1 / 21$ \\
\hline & & & $20-30 \%$ & $10.6 \pm 0.3$ & $0.166 \pm 0.003$ & $-0.415 \pm 0.005$ & $0.119 \pm 0.003$ & $1 / 21$ \\
\hline & & & $30-40 \%$ & $10.3 \pm 0.3$ & $0.162 \pm 0.003$ & $-0.414 \pm 0.005$ & $0.115 \pm 0.003$ & $2 / 21$ \\
\hline & & & $40-50 \%$ & $9.3 \pm 0.2$ & $0.153 \pm 0.003$ & $-0.409 \pm 0.005$ & $0.114 \pm 0.003$ & $3 / 21$ \\
\hline & & & $50-60 \%$ & $8.8 \pm 0.2$ & $0.146 \pm 0.002$ & $-0.401 \pm 0.005$ & $0.113 \pm 0.003$ & $5 / 21$ \\
\hline & & & $60-70 \%$ & $8.7 \pm 0.2$ & $0.139 \pm 0.002$ & $-0.395 \pm 0.005$ & $0.111 \pm 0.003$ & $4 / 21$ \\
\hline & & & $70-80 \%$ & $8.6 \pm 0.2$ & $0.132 \pm 0.002$ & $-0.387 \pm 0.005$ & $0.109 \pm 0.003$ & $18 / 21$ \\
\hline \multirow[t]{9}{*}{ Figure 4(c) } & $K^{-}$ & 19.6 & $0-5 \%$ & $21.1 \pm 1.1$ & $0.165 \pm 0.003$ & $-0.034 \pm 0.005$ & $0.124 \pm 0.005$ & $2 / 21$ \\
\hline & & $|y|<0.1$ & $5-10 \%$ & $19.3 \pm 0.9$ & $0.161 \pm 0.003$ & $-0.030 \pm 0.005$ & $0.121 \pm 0.005$ & $2 / 21$ \\
\hline & & & $10-20 \%$ & $17.9 \pm 0.8$ & $0.155 \pm 0.003$ & $-0.018 \pm 0.004$ & $0.119 \pm 0.005$ & $3 / 21$ \\
\hline & & & $20-30 \%$ & $16.5 \pm 0.7$ & $0.151 \pm 0.003$ & $-0.016 \pm 0.004$ & $0.115 \pm 0.005$ & $7 / 21$ \\
\hline & & & $30-40 \%$ & $14.4 \pm 0.5$ & $0.144 \pm 0.002$ & $-0.009 \pm 0.004$ & $0.114 \pm 0.005$ & $12 / 21$ \\
\hline & & & $40-50 \%$ & $13.3 \pm 0.4$ & $0.133 \pm 0.002$ & $0.012 \pm 0.004$ & $0.106 \pm 0.005$ & $12 / 20$ \\
\hline & & & $50-60 \%$ & $13.1 \pm 0.4$ & $0.126 \pm 0.002$ & $0.016 \pm 0.004$ & $0.103 \pm 0.005$ & $18 / 20$ \\
\hline & & & $60-70 \%$ & $11.0 \pm 0.3$ & $0.111 \pm 0.002$ & $0.032 \pm 0.005$ & $0.102 \pm 0.005$ & $30 / 18$ \\
\hline & & & $70-80 \%$ & $10.3 \pm 0.3$ & $0.105 \pm 0.002$ & $0.044 \pm 0.005$ & $0.102 \pm 0.005$ & $25 / 16$ \\
\hline \multirow[t]{9}{*}{ Figure 4(d) } & $K^{+}$ & 19.6 & $0-5 \%$ & $22.7 \pm 1.2$ & $0.172 \pm 0.003$ & $-0.045 \pm 0.005$ & $0.132 \pm 0.005$ & $1 / 21$ \\
\hline & & $|y|<0.1$ & $5-10 \%$ & $19.2 \pm 0.9$ & $0.168 \pm 0.003$ & $-0.034 \pm 0.005$ & $0.128 \pm 0.005$ & $3 / 21$ \\
\hline & & & $10-20 \%$ & $17.2 \pm 0.7$ & $0.164 \pm 0.003$ & $-0.030 \pm 0.005$ & $0.119 \pm 0.005$ & $3 / 21$ \\
\hline & & & $20-30 \%$ & $15.6 \pm 0.6$ & $0.159 \pm 0.003$ & $-0.026 \pm 0.004$ & $0.115 \pm 0.005$ & $5 / 21$ \\
\hline & & & $30-40 \%$ & $15.1 \pm 0.6$ & $0.151 \pm 0.003$ & $-0.020 \pm 0.004$ & $0.109 \pm 0.005$ & $15 / 21$ \\
\hline & & & $40-50 \%$ & $14.4 \pm 0.5$ & $0.145 \pm 0.002$ & $-0.017 \pm 0.004$ & $0.105 \pm 0.005$ & $14 / 20$ \\
\hline & & & $50-60 \%$ & $12.5 \pm 0.3$ & $0.141 \pm 0.002$ & $-0.011 \pm 0.004$ & $0.102 \pm 0.005$ & $23 / 20$ \\
\hline & & & $60-70 \%$ & $9.9 \pm 0.2$ & $0.127 \pm 0.002$ & $-0.006 \pm 0.004$ & $0.099 \pm 0.004$ & $15 / 18$ \\
\hline & & & $70-80 \%$ & $7.9 \pm 0.2$ & $0.115 \pm 0.002$ & $0.007 \pm 0.004$ & $0.093 \pm 0.004$ & $10 / 17$ \\
\hline
\end{tabular}


TABLE 4: Continued.

\begin{tabular}{|c|c|c|c|c|c|c|c|c|}
\hline Figure & Particle & $\sqrt{s_{N N}}(\mathrm{GeV})$ & Selection & $n$ & $T_{0}(\mathrm{GeV})$ & $a_{0}$ & $\left\langle\beta_{t}\right\rangle(c)$ & $\chi^{2} /$ ndof \\
\hline \multirow[t]{9}{*}{ Figure 4(e) } & $\bar{p}$ & 19.6 & $0-5 \%$ & $15.3 \pm 0.6$ & $0.132 \pm 0.002$ & $0.001 \pm 0.004$ & $0.367 \pm 0.008$ & $5 / 17$ \\
\hline & & $|y|<0.1$ & $5-10 \%$ & $14.3 \pm 0.5$ & $0.126 \pm 0.002$ & $0.003 \pm 0.004$ & $0.353 \pm 0.008$ & $9 / 17$ \\
\hline & & & $10-20 \%$ & $13.2 \pm 0.4$ & $0.123 \pm 0.002$ & $0.006 \pm 0.004$ & $0.341 \pm 0.008$ & $5 / 19$ \\
\hline & & & $20-30 \%$ & $12.8 \pm 0.3$ & $0.120 \pm 0.002$ & $0.009 \pm 0.004$ & $0.328 \pm 0.008$ & $3 / 19$ \\
\hline & & & $30-40 \%$ & $12.4 \pm 0.3$ & $0.111 \pm 0.002$ & $0.013 \pm 0.004$ & $0.315 \pm 0.008$ & $5 / 20$ \\
\hline & & & $40-50 \%$ & $11.0 \pm 0.3$ & $0.106 \pm 0.002$ & $0.014 \pm 0.004$ & $0.305 \pm 0.008$ & $7 / 20$ \\
\hline & & & $50-60 \%$ & $10.2 \pm 0.3$ & $0.094 \pm 0.001$ & $0.015 \pm 0.004$ & $0.299 \pm 0.008$ & $11 / 20$ \\
\hline & & & $60-70 \%$ & $9.7 \pm 0.2$ & $0.086 \pm 0.001$ & $0.017 \pm 0.004$ & $0.301 \pm 0.008$ & $9 / 18$ \\
\hline & & & $70-80 \%$ & $9.5 \pm 0.2$ & $0.080 \pm 0.001$ & $0.019 \pm 0.004$ & $0.284 \pm 0.007$ & $11 / 17$ \\
\hline \multirow[t]{9}{*}{ Figure 4(f) } & $p$ & 19.6 & $0-5 \%$ & $15.9 \pm 0.6$ & $0.133 \pm 0.002$ & $-0.002 \pm 0.004$ & $0.355 \pm 0.008$ & $7 / 24$ \\
\hline & & $|y|<0.1$ & $5-10 \%$ & $14.4 \pm 0.5$ & $0.132 \pm 0.002$ & $0.001 \pm 0.004$ & $0.345 \pm 0.008$ & $6 / 20$ \\
\hline & & & $10-20 \%$ & $13.3 \pm 0.4$ & $0.129 \pm 0.002$ & $0.005 \pm 0.004$ & $0.339 \pm 0.008$ & $5 / 18$ \\
\hline & & & $20-30 \%$ & $12.7 \pm 0.3$ & $0.122 \pm 0.002$ & $0.007 \pm 0.004$ & $0.328 \pm 0.008$ & $9 / 18$ \\
\hline & & & $30-40 \%$ & $12.3 \pm 0.3$ & $0.117 \pm 0.002$ & $0.010 \pm 0.004$ & $0.316 \pm 0.008$ & $8 / 18$ \\
\hline & & & $40-50 \%$ & $11.9 \pm 0.3$ & $0.116 \pm 0.002$ & $0.013 \pm 0.004$ & $0.305 \pm 0.008$ & $12 / 18$ \\
\hline & & & $50-60 \%$ & $11.3 \pm 0.3$ & $0.109 \pm 0.002$ & $0.015 \pm 0.004$ & $0.286 \pm 0.007$ & $15 / 18$ \\
\hline & & & $60-70 \%$ & $10.6 \pm 0.3$ & $0.104 \pm 0.002$ & $0.017 \pm 0.004$ & $0.280 \pm 0.007$ & $16 / 18$ \\
\hline & & & $70-80 \%$ & $9.8 \pm 0.2$ & $0.095 \pm 0.002$ & $0.019 \pm 0.004$ & $0.269 \pm 0.007$ & $13 / 18$ \\
\hline
\end{tabular}

In order to represent the chaos degree of the final state particle information, we show the dependence of pseudoentropy $S_{\text {hadron }}^{\prime}$ on $\sqrt{s_{N N}}$ and centrality in Figure 15, where panels (a)-(f) are orderly for the results of $\pi^{-}, \pi^{+}, K^{-}, K^{+}$, $\bar{p}$, and $p$. One can see that $S_{\text {hadron }}^{\prime}$ of the six kinds of hadrons in different centrality intervals increases with the increase of $\sqrt{s_{N N}}$. In particular, for $\pi^{\mp}$ and $K^{\mp}$ and in central collisions, $S_{\text {hadron }}^{\prime}$ increases quickly and then slowly with the increase of $\sqrt{s_{N N}}$, and the boundary energy is $7.7 \mathrm{GeV}$. From central to peripheral collisions, $S_{\text {hadron }}^{\prime}$ decreases gradually. The chaos degree of particle information at low energy is less than that at high energy. In addition, the chaos degree in central collisions is larger than that in peripheral collisions. Observing the values of $S_{\text {hadron }}^{\prime}$, one can clearly find that the order of chaos degree is $\bar{p}(p)>K^{\mp}>\pi^{\mp}$.

In the above discussion, we have used a particular parameterization for the $p_{T}$ distribution and obtained the tendencies of parameters, but many other parameterizations are available and used in the field. Our conclusions on the behaviors of main parameters $n$ [or $q=(n+1) / n$ ], $T_{0}$, and $\left\langle\beta_{t}\right\rangle$ with increasing the collision energy, event centrality, and particle mass are similar to others if one uses different parameterizations [48], though the absolute sizes may be different. The parameter $a_{0}$ is a new one introduced by us recently [45], which is hard to compare with others at present due to the lack of related work, though the relative sizes of $a_{0}$ on particle mass are understandable. The parameterization used in this paper is also suitable for smaller system studied in our previous work $[45,46]$, where the dependences of parameters on the collision energy, event centrality (or multiplicity), and particle mass are similar, which is not very sensitive to the system size.

3.3. Further Discussions. We now discuss this topic further. As we know, high-energy collisions contain abundant information which are related to the productions of QGP and particles [55-62]. Of course, after a very short time, QGP finally decays into particles. The final-state products are naturally various kinds of particles. Indeed, understanding the characteristics of particle productions is particularly necessary for researchers to study the evolution of collision system and interactions among various particles [63-70]. By studying the particle spectra, this paper has extracted some quantities.

From the above comparison, one can see that this paper has used the transverse mass which contains the average transverse flow velocity $\left\langle\beta_{t}\right\rangle$ through the Lorentz-like transformation, $m^{\prime}{ }_{T} \longrightarrow\left\langle\gamma_{t}\right\rangle\left(m_{T}-p_{T}\left\langle\beta_{t}\right\rangle\right)$ [49]. Considering the influence of transverse flow, the effective temperature $T$ in Equation (1) has been changed to the thermal freeze-out temperature $T_{0}$ in Equation (2). In this way, $T_{0}$ and $\left\langle\beta_{t}\right\rangle$ can be directly extracted [49]. In this work, $T_{0}$ and $\left\langle\beta_{t}\right\rangle$ are quantities at the quark level, but not the quantities at the particle level. This renders that the values obtained at the two levels are different.

There is a relation between the power index $n$ and the entropy index $q, n=1 /(q-1)$. It shows indeed that a larger $n$ corresponds to a smaller $q$ that is also closer to 1 . As we know, an exact and concrete $q$ that separates an approximate equilibrium and nonequilibrium states is not available in literature. Empirically, we may use $q=1.25$, i.e., $n>4$, as the 
TABLe 5: Values of $n, T_{0}, a_{0},\left\langle\beta_{t}\right\rangle, \chi^{2}$, and ndof corresponding to the curves in Figure 5.

\begin{tabular}{|c|c|c|c|c|c|c|c|c|}
\hline Figure & Particle & $\sqrt{s_{N N}}(\mathrm{GeV})$ & Selection & $n$ & $T_{0}(\mathrm{GeV})$ & $a_{0}$ & $\left\langle\beta_{t}\right\rangle(c)$ & $\chi^{2} /$ ndof \\
\hline \multirow[t]{9}{*}{ Figure 5(a) } & $\pi^{-}$ & 27 & $0-5 \%$ & $11.8 \pm 0.3$ & $0.175 \pm 0.003$ & $-0.410 \pm 0.005$ & $0.121 \pm 0.003$ & $4 / 21$ \\
\hline & & $|y|<0.1$ & $5-10 \%$ & $10.8 \pm 0.3$ & $0.170 \pm 0.003$ & $-0.409 \pm 0.005$ & $0.119 \pm 0.003$ & $1 / 21$ \\
\hline & & & $10-20 \%$ & $10.1 \pm 0.3$ & $0.167 \pm 0.003$ & $-0.407 \pm 0.005$ & $0.118 \pm 0.003$ & $2 / 21$ \\
\hline & & & $20-30 \%$ & $9.5 \pm 0.2$ & $0.164 \pm 0.003$ & $-0.405 \pm 0.005$ & $0.116 \pm 0.003$ & $1 / 21$ \\
\hline & & & $30-40 \%$ & $8.9 \pm 0.2$ & $0.160 \pm 0.003$ & $-0.403 \pm 0.005$ & $0.112 \pm 0.003$ & $1 / 21$ \\
\hline & & & $40-50 \%$ & $8.4 \pm 0.2$ & $0.155 \pm 0.003$ & $-0.398 \pm 0.005$ & $0.109 \pm 0.003$ & $3 / 21$ \\
\hline & & & $50-60 \%$ & $8.1 \pm 0.2$ & $0.148 \pm 0.002$ & $-0.388 \pm 0.005$ & $0.107 \pm 0.003$ & $2 / 21$ \\
\hline & & & $60-70 \%$ & $7.7 \pm 0.2$ & $0.139 \pm 0.002$ & $-0.385 \pm 0.005$ & $0.106 \pm 0.003$ & $4 / 21$ \\
\hline & & & $70-80 \%$ & $7.6 \pm 0.2$ & $0.133 \pm 0.002$ & $-0.373 \pm 0.005$ & $0.105 \pm 0.003$ & $13 / 21$ \\
\hline \multirow[t]{9}{*}{ Figure 5(b) } & $\pi^{+}$ & 27 & $0-5 \%$ & $11.7 \pm 0.3$ & $0.186 \pm 0.003$ & $-0.425 \pm 0.005$ & $0.126 \pm 0.003$ & $14 / 21$ \\
\hline & & $|y|<0.1$ & $5-10 \%$ & $11.2 \pm 0.3$ & $0.180 \pm 0.003$ & $-0.424 \pm 0.005$ & $0.124 \pm 0.003$ & $6 / 21$ \\
\hline & & & $10-20 \%$ & $9.9 \pm 0.2$ & $0.172 \pm 0.003$ & $-0.421 \pm 0.005$ & $0.122 \pm 0.003$ & $3 / 21$ \\
\hline & & & $20-30 \%$ & $9.8 \pm 0.2$ & $0.168 \pm 0.003$ & $-0.416 \pm 0.005$ & $0.120 \pm 0.003$ & $1 / 21$ \\
\hline & & & $30-40 \%$ & $9.6 \pm 0.2$ & $0.164 \pm 0.003$ & $-0.415 \pm 0.005$ & $0.118 \pm 0.003$ & $1 / 21$ \\
\hline & & & $40-50 \%$ & $8.6 \pm 0.2$ & $0.156 \pm 0.003$ & $-0.409 \pm 0.005$ & $0.115 \pm 0.003$ & $1 / 21$ \\
\hline & & & $50-60 \%$ & $7.9 \pm 0.2$ & $0.149 \pm 0.002$ & $-0.402 \pm 0.005$ & $0.116 \pm 0.003$ & $4 / 21$ \\
\hline & & & $60-70 \%$ & $7.8 \pm 0.2$ & $0.142 \pm 0.002$ & $-0.396 \pm 0.005$ & $0.113 \pm 0.003$ & $4 / 21$ \\
\hline & & & $70-80 \%$ & $7.3 \pm 0.2$ & $0.135 \pm 0.002$ & $-0.395 \pm 0.005$ & $0.112 \pm 0.003$ & $9 / 21$ \\
\hline \multirow[t]{9}{*}{ Figure 5(c) } & $K^{-}$ & 27 & $0-5 \%$ & $21.0 \pm 1.0$ & $0.166 \pm 0.003$ & $-0.038 \pm 0.005$ & $0.136 \pm 0.005$ & $12 / 20$ \\
\hline & & $|y|<0.1$ & $5-10 \%$ & $18.5 \pm 0.8$ & $0.164 \pm 0.003$ & $-0.036 \pm 0.005$ & $0.129 \pm 0.005$ & $4 / 21$ \\
\hline & & & $10-20 \%$ & $15.6 \pm 0.6$ & $0.158 \pm 0.003$ & $-0.028 \pm 0.004$ & $0.128 \pm 0.005$ & $4 / 21$ \\
\hline & & & $20-30 \%$ & $13.7 \pm 0.4$ & $0.152 \pm 0.003$ & $-0.019 \pm 0.004$ & $0.126 \pm 0.005$ & $5 / 21$ \\
\hline & & & $30-40 \%$ & $12.6 \pm 0.3$ & $0.148 \pm 0.002$ & $-0.010 \pm 0.004$ & $0.116 \pm 0.005$ & $5 / 21$ \\
\hline & & & $40-50 \%$ & $10.2 \pm 0.3$ & $0.137 \pm 0.002$ & $-0.002 \pm 0.004$ & $0.111 \pm 0.005$ & $6 / 21$ \\
\hline & & & $50-60 \%$ & $9.4 \pm 0.2$ & $0.128 \pm 0.002$ & $0.009 \pm 0.005$ & $0.110 \pm 0.005$ & $6 / 21$ \\
\hline & & & $60-70 \%$ & $8.9 \pm 0.2$ & $0.120 \pm 0.002$ & $0.014 \pm 0.004$ & $0.105 \pm 0.005$ & $10 / 21$ \\
\hline & & & $70-80 \%$ & $8.6 \pm 0.2$ & $0.111 \pm 0.002$ & $0.018 \pm 0.004$ & $0.105 \pm 0.005$ & $22 / 21$ \\
\hline \multirow[t]{9}{*}{ Figure 5(d) } & $K^{+}$ & 27 & $0-5 \%$ & $21.9 \pm 1.1$ & $0.175 \pm 0.003$ & $-0.050 \pm 0.005$ & $0.144 \pm 0.005$ & $12 / 21$ \\
\hline & & $|y|<0.1$ & $5-10 \%$ & $19.2 \pm 0.9$ & $0.171 \pm 0.003$ & $-0.035 \pm 0.005$ & $0.137 \pm 0.005$ & $7 / 21$ \\
\hline & & & $10-20 \%$ & $17.2 \pm 0.7$ & $0.167 \pm 0.003$ & $-0.029 \pm 0.004$ & $0.133 \pm 0.005$ & $7 / 21$ \\
\hline & & & $20-30 \%$ & $13.4 \pm 0.4$ & $0.162 \pm 0.003$ & $-0.026 \pm 0.004$ & $0.126 \pm 0.005$ & $3 / 21$ \\
\hline & & & $30-40 \%$ & $11.0 \pm 0.3$ & $0.152 \pm 0.003$ & $-0.022 \pm 0.004$ & $0.123 \pm 0.005$ & $3 / 21$ \\
\hline & & & $40-50 \%$ & $9.5 \pm 0.2$ & $0.143 \pm 0.002$ & $-0.019 \pm 0.004$ & $0.115 \pm 0.005$ & $4 / 21$ \\
\hline & & & $50-60 \%$ & $9.1 \pm 0.2$ & $0.138 \pm 0.002$ & $-0.015 \pm 0.004$ & $0.109 \pm 0.005$ & $5 / 21$ \\
\hline & & & $60-70 \%$ & $8.6 \pm 0.2$ & $0.129 \pm 0.002$ & $-0.012 \pm 0.004$ & $0.102 \pm 0.005$ & $6 / 21$ \\
\hline & & & $70-80 \%$ & $7.5 \pm 0.2$ & $0.119 \pm 0.002$ & $-0.004 \pm 0.004$ & $0.096 \pm 0.004$ & $13 / 21$ \\
\hline
\end{tabular}


TABLE 5: Continued.

\begin{tabular}{|c|c|c|c|c|c|c|c|c|}
\hline Figure & Particle & $\sqrt{s_{N N}}(\mathrm{GeV})$ & Selection & $n$ & $T_{0}(\mathrm{GeV})$ & $a_{0}$ & $\left\langle\beta_{t}\right\rangle(c)$ & $\chi^{2} /$ ndof \\
\hline \multirow[t]{9}{*}{ Figure 5(e) } & $\bar{p}$ & 27 & $0-5 \%$ & $14.6 \pm 0.5$ & $0.134 \pm 0.002$ & $-0.001 \pm 0.004$ & $0.379 \pm 0.008$ & $5 / 17$ \\
\hline & & $|y|<0.1$ & $5-10 \%$ & $14.0 \pm 0.4$ & $0.130 \pm 0.002$ & $0.003 \pm 0.004$ & $0.375 \pm 0.008$ & $4 / 17$ \\
\hline & & & $10-20 \%$ & $12.4 \pm 0.3$ & $0.127 \pm 0.002$ & $0.005 \pm 0.004$ & $0.356 \pm 0.008$ & $3 / 17$ \\
\hline & & & $20-30 \%$ & $11.3 \pm 0.3$ & $0.124 \pm 0.002$ & $0.008 \pm 0.004$ & $0.330 \pm 0.008$ & $2 / 17$ \\
\hline & & & $30-40 \%$ & $10.7 \pm 0.3$ & $0.115 \pm 0.002$ & $0.011 \pm 0.004$ & $0.328 \pm 0.008$ & $3 / 17$ \\
\hline & & & $40-50 \%$ & $9.8 \pm 0.2$ & $0.110 \pm 0.002$ & $0.015 \pm 0.004$ & $0.304 \pm 0.008$ & $3 / 17$ \\
\hline & & & $50-60 \%$ & $9.2 \pm 0.2$ & $0.097 \pm 0.002$ & $0.016 \pm 0.004$ & $0.307 \pm 0.008$ & $7 / 17$ \\
\hline & & & $60-70 \%$ & $8.9 \pm 0.2$ & $0.090 \pm 0.002$ & $0.018 \pm 0.004$ & $0.300 \pm 0.007$ & $10 / 17$ \\
\hline & & & $70-80 \%$ & $8.8 \pm 0.2$ & $0.084 \pm 0.001$ & $0.019 \pm 0.004$ & $0.285 \pm 0.007$ & $10 / 15$ \\
\hline \multirow[t]{9}{*}{ Figure 5(f) } & $p$ & 27 & $0-5 \%$ & $14.7 \pm 0.5$ & $0.136 \pm 0.002$ & $-0.005 \pm 0.004$ & $0.359 \pm 0.008$ & $6 / 18$ \\
\hline & & $|y|<0.1$ & $5-10 \%$ & $13.7 \pm 0.4$ & $0.133 \pm 0.002$ & $-0.003 \pm 0.004$ & $0.349 \pm 0.008$ & $6 / 18$ \\
\hline & & & $10-20 \%$ & $13.1 \pm 0.4$ & $0.130 \pm 0.002$ & $0.004 \pm 0.004$ & $0.339 \pm 0.008$ & $5 / 18$ \\
\hline & & & $20-30 \%$ & $11.6 \pm 0.3$ & $0.124 \pm 0.002$ & $0.005 \pm 0.004$ & $0.333 \pm 0.008$ & $6 / 18$ \\
\hline & & & $30-40 \%$ & $12.0 \pm 0.3$ & $0.119 \pm 0.002$ & $0.007 \pm 0.004$ & $0.328 \pm 0.008$ & $8 / 18$ \\
\hline & & & $40-50 \%$ & $12.1 \pm 0.3$ & $0.118 \pm 0.002$ & $0.011 \pm 0.004$ & $0.311 \pm 0.008$ & $13 / 18$ \\
\hline & & & $50-60 \%$ & $10.7 \pm 0.3$ & $0.109 \pm 0.002$ & $0.013 \pm 0.004$ & $0.298 \pm 0.007$ & $15 / 18$ \\
\hline & & & $60-70 \%$ & $10.0 \pm 0.3$ & $0.105 \pm 0.002$ & $0.016 \pm 0.004$ & $0.283 \pm 0.007$ & $20 / 18$ \\
\hline & & & $70-80 \%$ & $9.7 \pm 0.2$ & $0.100 \pm 0.002$ & $0.018 \pm 0.004$ & $0.264 \pm 0.007$ & $28 / 18$ \\
\hline
\end{tabular}

boundary between the approximate equilibrium and nonequilibrium states. The present work shows that $n>4$ in most cases. This means that the system in terms of quarks or partons discussed here is at the approximate equilibrium or local equilibriums. The concept of temperature can be approximately used.

At lower energy and in central collisions, the system consisted of quarks or partons is at larger degree of equilibrium. This is understood in view of the longer reaction time in lower collision energy and more participant partons in central collisions. These two factors can affect the system to be at more equilibrium due to more cascade collisions happening. At the present energy at the LHC, the system is approximately at the equilibrium. It is possible that at very high energy, the system will be at nonequilibrium due to very short reaction time. In most cases, the system in peripheral collisions is approximately at the equilibrium. It is not preclusive that in very peripheral collisions, the system is possible at nonequilibrium due to very less cascade collisions in very limited collision volume, where the multiplicity is also low.

There are possible scenarios for single, double, or multiple kinetic freeze-out in literature. The single (two or multiple) kinetic freeze-out means that a set (two or multiple sets) of parameters can fit the spectra. There is not a sold conclusion for a definite scenario. The present work shows that some parameters from different particle spectra are different. This reflects the scenario for multiple kinetic freeze-out in high-energy collisions. According to the present work, we may say that different particles are produced in the system at different times. The decay of the system is not all at once, but successive.
The value of $T_{0}$ reflects the excitation degree of the system at the parton level, which is somehow larger than that at the particle level, due to the former happening earlier. The higher the value of $T_{0}$ is, the higher the excitation degree of the system is, and the larger the deposited energy is. The value of $\left\langle\beta_{t}\right\rangle$ reflects impact and squeeze between the projectile and target at parton level. In high-energy collisions, a lot of energy are deposited in the process of parton interactions, though some partons go through the system as spectators.

Some soft excitation and hard scattering processes among partons have happened. The contribution of soft component from resonance in $\pi^{\mp}$ spectra is significantly larger than those in $K^{\mp}$ and $\bar{p}(p)$ spectra. This renders that the spectra of $\pi^{\mp}$ have a significant increase in low- $p_{T}$ region, comparing with the spectra of other particles. This change in low- $p_{T}$ region is mainly described by the value of $a_{0}$ in this work. A small $a_{0}$ describes a significant increase, and a large $a_{0}$ describes a decrease. Naturally, this change also affects the parameters $n, T_{0}$, and $\left\langle\beta_{t}\right\rangle$.

Indeed, for $\pi^{\mp}$ spectra in low- $p_{T}$ region, the production of resonances cannot be ignored. More contributions from the resonances in $\pi^{\mp}$ productions render that the shape of the spectra of the considered different kinds of particles is different, in particular, in low- $p_{T}$ region. This also renders the different sets of parameters for different particles. In addition, multiple kinetic freeze-out also results in multiple sets of parameters. The earlier the particles are emitted, the wider the spectrum is, and the higher the temperature is.

At given collision energy, the nuclear environments (the sizes of participant or spectator regions) in central and 
TABLe 6: Values of $n, T_{0}, a_{0},\left\langle\beta_{t}\right\rangle, \chi^{2}$, and ndof corresponding to the curves in Figure 6.

\begin{tabular}{|c|c|c|c|c|c|c|c|c|}
\hline Figure & Particle & $\sqrt{s_{N N}}(\mathrm{GeV})$ & Selection & $n$ & $T_{0}(\mathrm{GeV})$ & $a_{0}$ & $\left\langle\beta_{t}\right\rangle(c)$ & $\chi^{2} /$ ndof \\
\hline \multirow[t]{9}{*}{ Figure 6(a) } & $\pi^{-}$ & 39 & $0-5 \%$ & $10.1 \pm 0.3$ & $0.179 \pm 0.003$ & $-0.418 \pm 0.005$ & $0.122 \pm 0.003$ & $2 / 21$ \\
\hline & & $|y|<0.1$ & $5-10 \%$ & $9.2 \pm 0.2$ & $0.173 \pm 0.003$ & $-0.414 \pm 0.005$ & $0.120 \pm 0.003$ & $2 / 21$ \\
\hline & & & $10-20 \%$ & $8.8 \pm 0.2$ & $0.170 \pm 0.003$ & $-0.412 \pm 0.005$ & $0.118 \pm 0.003$ & $1 / 21$ \\
\hline & & & $20-30 \%$ & $8.3 \pm 0.2$ & $0.167 \pm 0.003$ & $-0.411 \pm 0.005$ & $0.115 \pm 0.003$ & $1 / 21$ \\
\hline & & & $30-40 \%$ & $7.9 \pm 0.2$ & $0.162 \pm 0.003$ & $-0.403 \pm 0.005$ & $0.113 \pm 0.003$ & $1 / 21$ \\
\hline & & & $40-50 \%$ & $7.6 \pm 0.2$ & $0.158 \pm 0.003$ & $-0.399 \pm 0.005$ & $0.107 \pm 0.003$ & $1 / 21$ \\
\hline & & & $50-60 \%$ & $7.1 \pm 0.2$ & $0.150 \pm 0.003$ & $-0.394 \pm 0.005$ & $0.106 \pm 0.003$ & $1 / 21$ \\
\hline & & & $60-70 \%$ & $6.7 \pm 0.1$ & $0.142 \pm 0.002$ & $-0.391 \pm 0.005$ & $0.105 \pm 0.003$ & $3 / 21$ \\
\hline & & & $70-80 \%$ & $6.5 \pm 0.1$ & $0.136 \pm 0.002$ & $-0.384 \pm 0.005$ & $0.104 \pm 0.003$ & $6 / 21$ \\
\hline \multirow[t]{9}{*}{ Figure 6(b) } & $\pi^{+}$ & 39 & $0-5 \%$ & $9.2 \pm 0.2$ & $0.190 \pm 0.003$ & $-0.428 \pm 0.005$ & $0.126 \pm 0.003$ & $28 / 21$ \\
\hline & & $|y|<0.1$ & $5-10 \%$ & $8.8 \pm 0.2$ & $0.183 \pm 0.003$ & $-0.426 \pm 0.005$ & $0.125 \pm 0.003$ & $19 / 21$ \\
\hline & & & $10-20 \%$ & $8.4 \pm 0.2$ & $0.175 \pm 0.003$ & $-0.422 \pm 0.005$ & $0.123 \pm 0.003$ & $5 / 21$ \\
\hline & & & $20-30 \%$ & $8.1 \pm 0.2$ & $0.170 \pm 0.003$ & $-0.418 \pm 0.005$ & $0.122 \pm 0.003$ & $2 / 21$ \\
\hline & & & $30-40 \%$ & $7.9 \pm 0.2$ & $0.166 \pm 0.003$ & $-0.417 \pm 0.005$ & $0.119 \pm 0.003$ & $1 / 21$ \\
\hline & & & $40-50 \%$ & $7.3 \pm 0.2$ & $0.158 \pm 0.003$ & $-0.411 \pm 0.005$ & $0.118 \pm 0.003$ & $2 / 21$ \\
\hline & & & $50-60 \%$ & $6.9 \pm 0.1$ & $0.151 \pm 0.003$ & $-0.406 \pm 0.005$ & $0.114 \pm 0.003$ & $1 / 21$ \\
\hline & & & $60-70 \%$ & $6.5 \pm 0.1$ & $0.144 \pm 0.002$ & $-0.403 \pm 0.005$ & $0.112 \pm 0.003$ & $3 / 21$ \\
\hline & & & $70-80 \%$ & $6.1 \pm 0.1$ & $0.137 \pm 0.002$ & $-0.399 \pm 0.005$ & $0.111 \pm 0.003$ & $5 / 21$ \\
\hline \multirow[t]{9}{*}{ Figure 6(c) } & $K^{-}$ & 39 & $0-5 \%$ & $19.8 \pm 0.9$ & $0.171 \pm 0.003$ & $-0.039 \pm 0.005$ & $0.164 \pm 0.005$ & $6 / 21$ \\
\hline & & $|y|<0.1$ & $5-10 \%$ & $17.8 \pm 0.7$ & $0.167 \pm 0.003$ & $-0.033 \pm 0.005$ & $0.152 \pm 0.005$ & $5 / 21$ \\
\hline & & & $10-20 \%$ & $15.6 \pm 0.6$ & $0.165 \pm 0.003$ & $-0.024 \pm 0.004$ & $0.142 \pm 0.005$ & $3 / 21$ \\
\hline & & & $20-30 \%$ & $14.4 \pm 0.5$ & $0.161 \pm 0.003$ & $-0.019 \pm 0.004$ & $0.137 \pm 0.005$ & $5 / 21$ \\
\hline & & & $30-40 \%$ & $11.4 \pm 0.3$ & $0.152 \pm 0.003$ & $-0.009 \pm 0.004$ & $0.133 \pm 0.005$ & $5 / 21$ \\
\hline & & & $40-50 \%$ & $9.7 \pm 0.2$ & $0.139 \pm 0.002$ & $-0.005 \pm 0.004$ & $0.130 \pm 0.005$ & $7 / 21$ \\
\hline & & & $50-60 \%$ & $9.0 \pm 0.2$ & $0.132 \pm 0.002$ & $-0.002 \pm 0.004$ & $0.126 \pm 0.005$ & $9 / 21$ \\
\hline & & & $60-70 \%$ & $7.8 \pm 0.2$ & $0.124 \pm 0.002$ & $0.008 \pm 0.004$ & $0.123 \pm 0.005$ & $11 / 21$ \\
\hline & & & $70-80 \%$ & $7.0 \pm 0.1$ & $0.115 \pm 0.002$ & $0.016 \pm 0.004$ & $0.113 \pm 0.005$ & $15 / 21$ \\
\hline \multirow[t]{9}{*}{ Figure 6(d) } & $K^{+}$ & 39 & $0-5 \%$ & $20.6 \pm 1.0$ & $0.177 \pm 0.003$ & $-0.051 \pm 0.005$ & $0.150 \pm 0.005$ & $8 / 21$ \\
\hline & & $|y|<0.1$ & $5-10 \%$ & $18.7 \pm 0.8$ & $0.175 \pm 0.003$ & $-0.042 \pm 0.005$ & $0.145 \pm 0.005$ & $4 / 21$ \\
\hline & & & $10-20 \%$ & $15.3 \pm 0.6$ & $0.169 \pm 0.003$ & $-0.030 \pm 0.005$ & $0.138 \pm 0.005$ & $3 / 21$ \\
\hline & & & $20-30 \%$ & $11.4 \pm 0.3$ & $0.164 \pm 0.003$ & $-0.029 \pm 0.004$ & $0.128 \pm 0.005$ & $3 / 21$ \\
\hline & & & $30-40 \%$ & $10.3 \pm 0.3$ & $0.157 \pm 0.003$ & $-0.024 \pm 0.004$ & $0.122 \pm 0.005$ & $3 / 21$ \\
\hline & & & $40-50 \%$ & $7.9 \pm 0.2$ & $0.145 \pm 0.002$ & $-0.021 \pm 0.004$ & $0.119 \pm 0.005$ & $3 / 21$ \\
\hline & & & $50-60 \%$ & $7.6 \pm 0.2$ & $0.138 \pm 0.002$ & $-0.019 \pm 0.004$ & $0.117 \pm 0.005$ & $7 / 21$ \\
\hline & & & $60-70 \%$ & $7.1 \pm 0.2$ & $0.132 \pm 0.002$ & $-0.008 \pm 0.004$ & $0.115 \pm 0.005$ & $5 / 21$ \\
\hline & & & $70-80 \%$ & $6.1 \pm 0.1$ & $0.121 \pm 0.002$ & $-0.003 \pm 0.004$ & $0.106 \pm 0.005$ & $9 / 21$ \\
\hline
\end{tabular}


TABLE 6: Continued.

\begin{tabular}{|c|c|c|c|c|c|c|c|c|}
\hline Figure & Particle & $\sqrt{s_{N N}}(\mathrm{GeV})$ & Selection & $n$ & $T_{0}(\mathrm{GeV})$ & $a_{0}$ & $\left\langle\beta_{t}\right\rangle(c)$ & $\chi^{2} /$ ndof \\
\hline \multirow[t]{9}{*}{ Figure 6(e) } & \multirow[t]{9}{*}{$\bar{p}$} & 39 & $0-5 \%$ & $13.1 \pm 0.4$ & $0.135 \pm 0.002$ & $-0.002 \pm 0.004$ & $0.387 \pm 0.008$ & $7 / 18$ \\
\hline & & $|y|<0.1$ & $5-10 \%$ & $12.7 \pm 0.3$ & $0.130 \pm 0.002$ & $0.003 \pm 0.004$ & $0.378 \pm 0.008$ & $5 / 18$ \\
\hline & & & $10-20 \%$ & $11.9 \pm 0.3$ & $0.128 \pm 0.002$ & $0.004 \pm 0.004$ & $0.370 \pm 0.008$ & $4 / 18$ \\
\hline & & & $20-30 \%$ & $11.3 \pm 0.3$ & $0.124 \pm 0.002$ & $0.005 \pm 0.004$ & $0.340 \pm 0.008$ & $5 / 18$ \\
\hline & & & $30-40 \%$ & $10.5 \pm 0.3$ & $0.117 \pm 0.002$ & $0.007 \pm 0.004$ & $0.334 \pm 0.008$ & $3 / 18$ \\
\hline & & & $40-50 \%$ & $9.0 \pm 0.2$ & $0.111 \pm 0.002$ & $0.008 \pm 0.004$ & $0.322 \pm 0.008$ & $3 / 18$ \\
\hline & & & $50-60 \%$ & $8.7 \pm 0.2$ & $0.101 \pm 0.002$ & $0.010 \pm 0.004$ & $0.316 \pm 0.008$ & $5 / 18$ \\
\hline & & & $60-70 \%$ & $8.1 \pm 0.2$ & $0.091 \pm 0.002$ & $0.012 \pm 0.004$ & $0.314 \pm 0.008$ & $7 / 18$ \\
\hline & & & $70-80 \%$ & $8.0 \pm 0.2$ & $0.088 \pm 0.001$ & $0.015 \pm 0.004$ & $0.290 \pm 0.007$ & $7 / 18$ \\
\hline \multirow[t]{9}{*}{ Figure 6(f) } & \multirow[t]{9}{*}{$p$} & 39 & $0-5 \%$ & $13.9 \pm 0.4$ & $0.139 \pm 0.002$ & $-0.007 \pm 0.004$ & $0.376 \pm 0.008$ & $3 / 17$ \\
\hline & & $|y|<0.1$ & $5-10 \%$ & $13.1 \pm 0.4$ & $0.135 \pm 0.002$ & $-0.004 \pm 0.004$ & $0.365 \pm 0.008$ & $5 / 17$ \\
\hline & & & $10-20 \%$ & $11.8 \pm 0.3$ & $0.133 \pm 0.002$ & $0.001 \pm 0.004$ & $0.350 \pm 0.008$ & $3 / 17$ \\
\hline & & & $20-30 \%$ & $10.3 \pm 0.3$ & $0.127 \pm 0.002$ & $0.003 \pm 0.004$ & $0.341 \pm 0.008$ & $4 / 17$ \\
\hline & & & $30-40 \%$ & $9.9 \pm 0.2$ & $0.121 \pm 0.002$ & $0.005 \pm 0.004$ & $0.332 \pm 0.008$ & $8 / 17$ \\
\hline & & & $40-50 \%$ & $9.5 \pm 0.2$ & $0.120 \pm 0.002$ & $0.007 \pm 0.004$ & $0.310 \pm 0.008$ & $6 / 17$ \\
\hline & & & $50-60 \%$ & $8.9 \pm 0.2$ & $0.111 \pm 0.002$ & $0.009 \pm 0.004$ & $0.303 \pm 0.008$ & $8 / 17$ \\
\hline & & & $60-70 \%$ & $8.5 \pm 0.2$ & $0.104 \pm 0.002$ & $0.011 \pm 0.004$ & $0.288 \pm 0.007$ & $9 / 17$ \\
\hline & & & $70-80 \%$ & $9.1 \pm 0.2$ & $0.100 \pm 0.002$ & $0.013 \pm 0.004$ & $0.276 \pm 0.007$ & $20 / 17$ \\
\hline
\end{tabular}

peripheral $\mathrm{Au}-\mathrm{Au}$ collisions have some influences on the transverse momentum spectra. We attribute this phenomenon to the hadronic cascade collisions (or multiple scattering) in hot and dense participant zone. The larger number of participants in central collisions means more cascade collisions and, hence, the larger transverse momentum and temperature. In the considered transverse momentum region, the changing laws of transverse momentum spectra for given particles are independent of isospin. Compared with strong interactions, contribution of electromagnetic interactions is very small and negligible.

The purpose of the RHIC-BES program is to search for the critical point in the QCD phase diagram. In the RHICBES and the related AGS energy regions, from the energy dependences of related parameters in the available central collisions, we found that $T_{0}$ and $S_{\text {hadron }}^{\prime}$ increase quickly and then slowly at around $7.7 \mathrm{GeV}$ with the increase of collision energy. This special energy is the knee point of the equation of state (EoS) of the hot and dense matter, which is related to the critical energy, if not equal. Meanwhile, we found that $a_{0}$ increases and then decreases at around $7.7 \mathrm{GeV}$ in the available central collisions. Generally, different parameters may reveal different knee points. The most probable knee point from the energy dependences of various parameters can be regarded as the critical point.

In the available central collisions, both the boundary energies of the rapid-slow increase in the energy dependence of $T_{0}$ and $S_{\text {hadron }}^{\prime}$ and of the increase-decrease change in the energy dependence of $a_{0}$ are $7.7 \mathrm{GeV}$. We argue that this energy is possibly the critical energy of deconfinement phase transition from hadronic matter to QGP. Because of the complexity of the collision process, the conclusion about the critical energy in this work is not solid. A shift of 2$3 \mathrm{GeV}$ is possible. To make a solid conclusion, one needs a comprehensive analysis of various parameters. At least, we may state that the interaction mechanisms or strengths at the energy below and above about $7.7 \mathrm{GeV}$ are different.

\section{Summary and Conclusions}

In this paper, the TP-like function has been used to analyze the transverse momentum spectra of the charged particles $\left[\pi^{\mp}, K^{\mp}\right.$, and $\left.\bar{p}(p)\right]$ produced in $\mathrm{Au}-\mathrm{Au}$ collisions at the RHIC-BES energies and the related AGS energies. The contribution of each constituent quark to the transverse momentum of charged particle is assumed to satisfy the TP-like function. Each constituent quark is also regarded as an energy resource. Therefore, the transverse momentum spectrum of mesons (baryons) is the convolution of two (three) TP-like functions. According to the fitting results, we have obtained four parameters: the power index $n$, the thermal freeze-out temperature $T_{0}$, the correction index $a_{0}$, and the average transverse flow velocity $\left\langle\beta_{t}\right\rangle$. The dependences of these parameters on collision energy and centrality have been also investigated.

It is obvious that with the increase of collision energy, $n$ decreases, which indicates that the collision system is farther away from the equilibrium, though it is still approximately at the equilibrium, at higher energy. In other words, at lower energy, the longer the collision evolves, the closer to the equilibrium the system is. More importantly, with the increase of collision energy, $T_{0}$ extracted from meson 


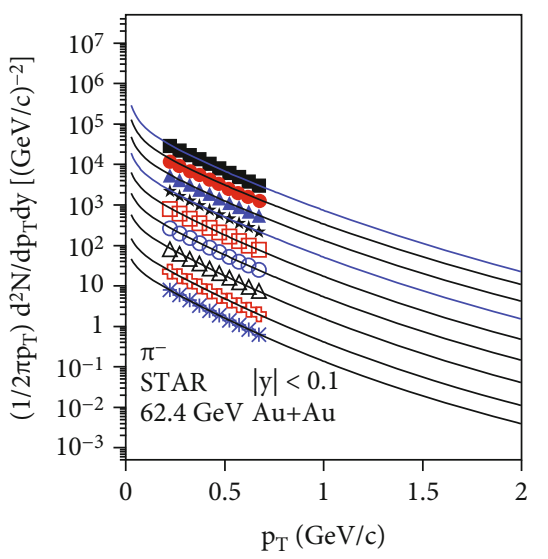

- $0-5 \%(\times 128)$ - $5-10 \%(\times 64)$

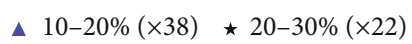

$\square 30-40 \%(\times 12) \quad \circ \quad 40-50 \%(\times 6)$

$\triangle 50-60 \%(\times 3)$ ↔ $60-70 \%(\times 1.5)$

* $70-80 \%$

(a)

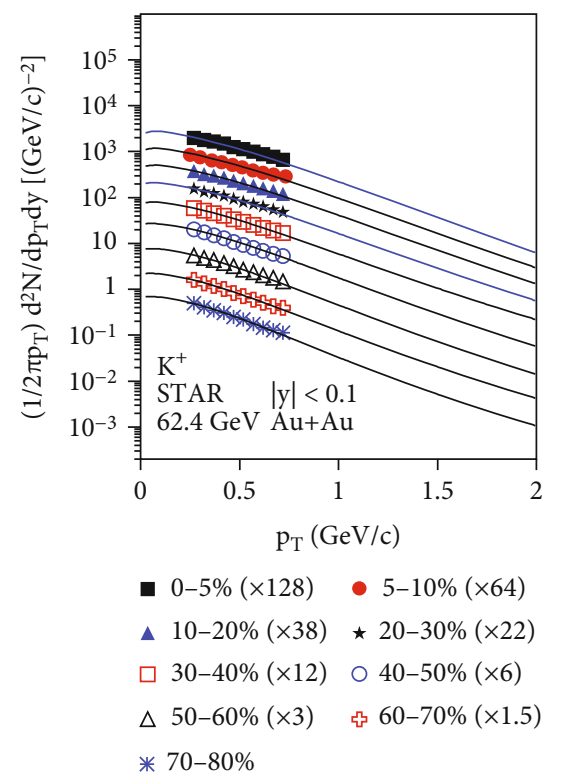

(d)

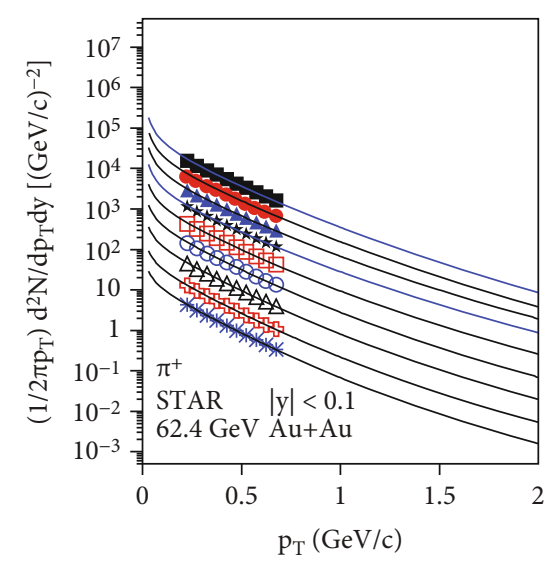

- $0-5 \%(\times 128)$ - $5-10 \%(\times 64)$

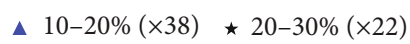

$\square 30-40 \%(\times 12) \quad \circ 40-50 \%(\times 6)$

$\triangle 50-60 \%(\times 3)$ ↔ $60-70 \%(\times 1.5)$

* $70-80 \%$

(b)

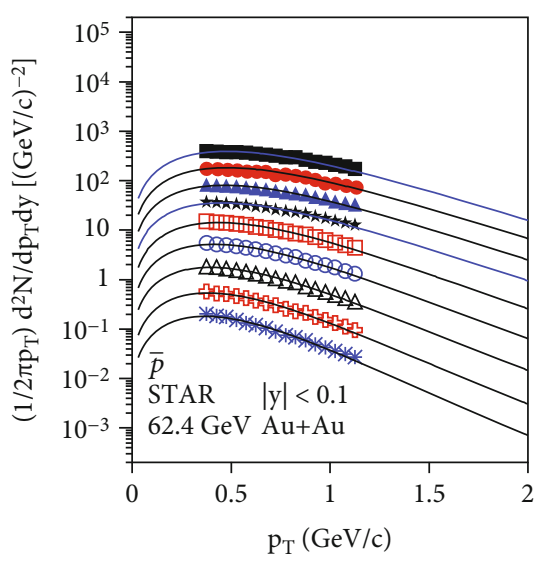

- $0-5 \%(\times 128)$ - $5-10 \%(\times 64)$

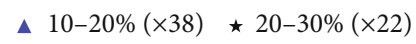

$\square 30-40 \%(\times 12) \quad$ ○ $40-50 \%(\times 6)$

$\triangle 50-60 \%(\times 3)$ ↔ $60-70 \%(\times 1.5)$

* $70-80 \%$

(e)

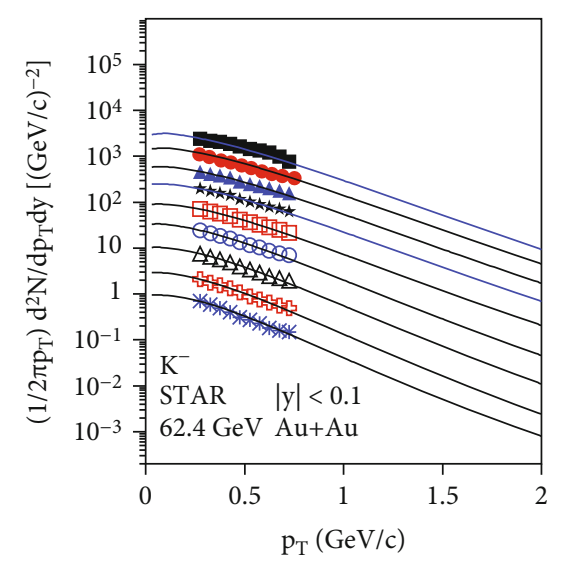

- $0-5 \%(\times 128)$ - $5-10 \%(\times 64)$

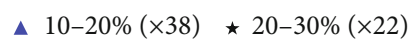

$\square 30-40 \%(\times 12) \quad \circ 40-50 \%(\times 6)$

$\triangle 50-60 \%(\times 3)$ ↔ $60-70 \%(\times 1.5)$

* $70-80 \%$

(c)

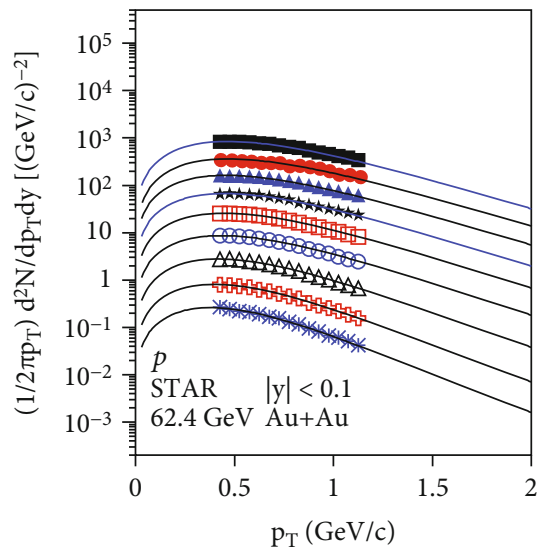

- $0-5 \%(\times 128)$ - $5-10 \%(\times 64)$

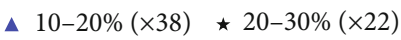

$\square$ 30-40\% $(\times 12) \quad$ ○ $40-50 \%(\times 6)$

$\triangle 50-60 \%(\times 3)$ ↔ $60-70 \%(\times 1.5)$

* $70-80 \%$

(f)

FIGURE 7: Same as Figure 1, but showing the results for $62.4 \mathrm{GeV} \mathrm{Au-Au} \mathrm{collisions.} \mathrm{The} \mathrm{symbols} \mathrm{represent} \mathrm{the} \mathrm{STAR} \mathrm{data} \mathrm{[35].}$ 


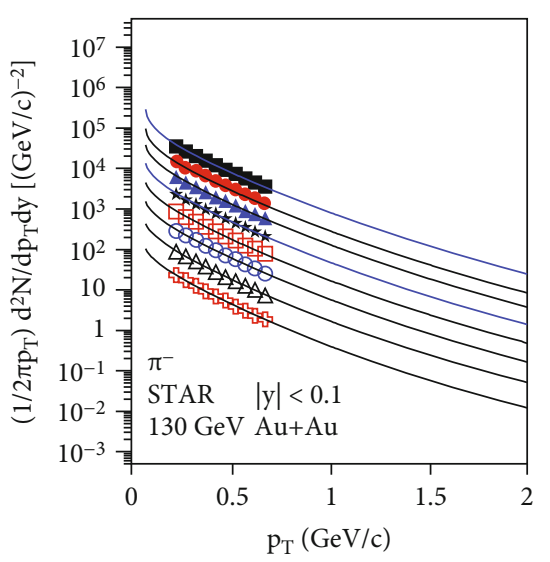

- $0-6 \%(\times 96)$ - $6-11 \%(\times 48)$

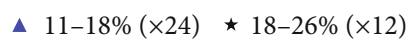

$\square 26-34 \%(\times 6) \quad$ ○ $34-45 \%(\times 3)$

$\triangle 45-58 \%(\times 1.5)$ њ $58-85 \%$

(a)

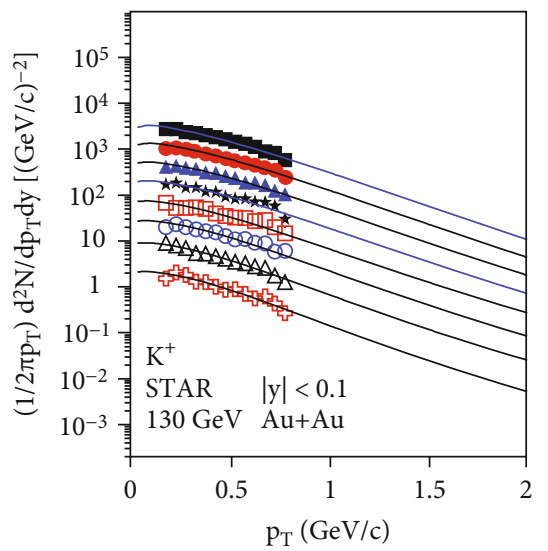

- $0-6 \%(\times 96)$ - $6-11 \%(\times 48)$

\ $11-18 \%(\times 24) \star 18-26 \%(\times 12)$

$\square 26-34 \%(\times 6) \quad$ ○ $34-45 \%(\times 3)$

$\triangle 45-58 \%(\times 1.5)$ फ $58-85 \%$

(d)

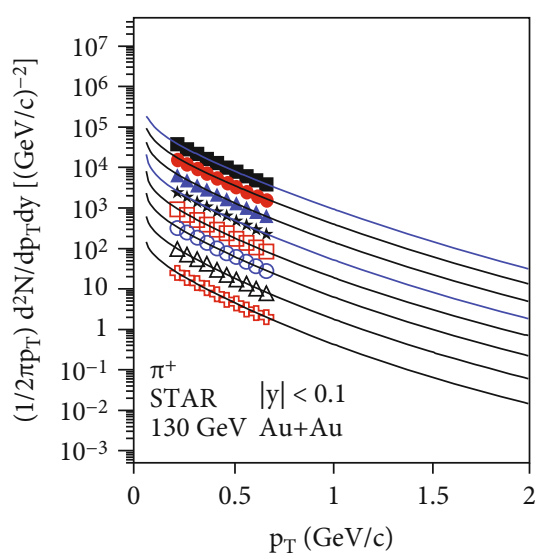

- $0-6 \%(\times 96)$ - $6-11 \%(\times 48)$

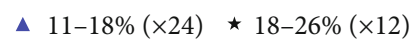

$\square 26-34 \%(\times 6) \quad$ ○ $34-45 \%(\times 3)$

$\triangle 45-58 \%(\times 1.5)$ ๒ $58-85 \%$

(b)

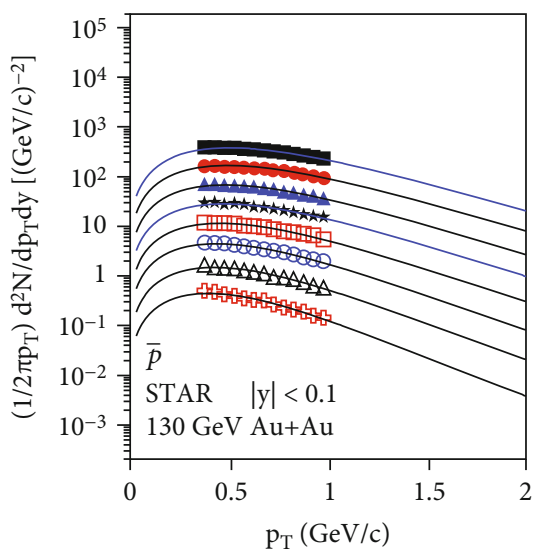

- $0-6 \%(\times 96)$ - $6-11 \%(\times 48)$

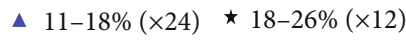

$\square 26-34 \%(\times 6) \quad \bigcirc 34-45 \%(\times 3)$

$\triangle 45-58 \%(\times 1.5)$ њ $58-85 \%$

(e)

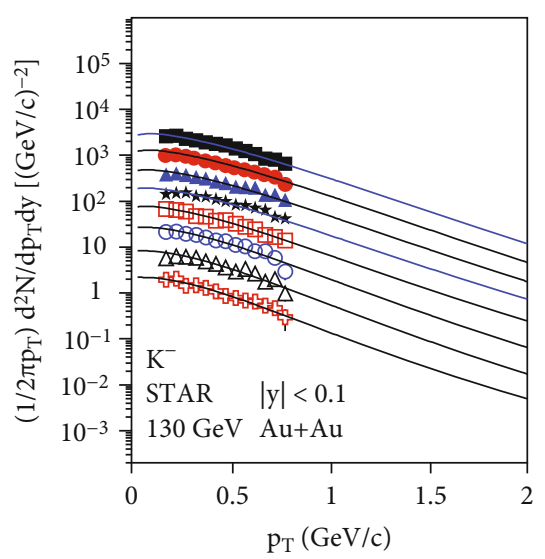

- $0-6 \%(\times 96)$ - $6-11 \%(\times 48)$

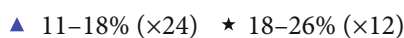

$\square 26-34 \%(\times 6) \quad$ ○ $34-45 \%(\times 3)$

$\triangle 45-58 \%(\times 1.5)$ њ $58-85 \%$

(c)

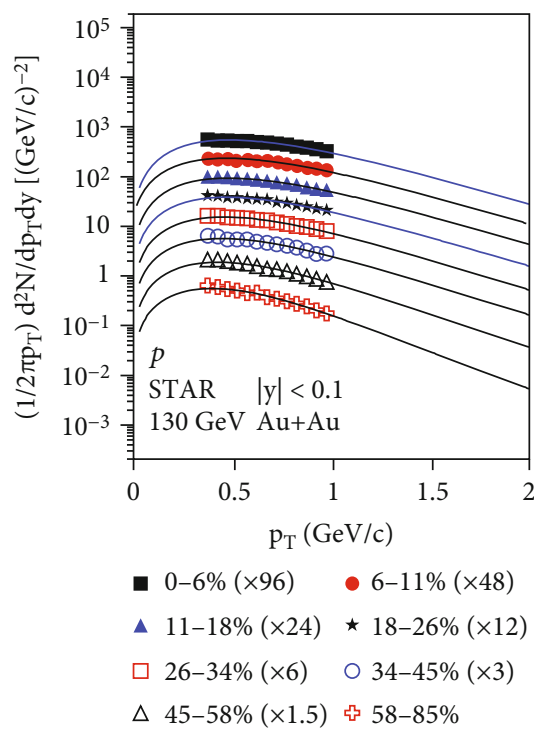

(f)

Figure 8: Same as Figure 1, but showing the results for $130 \mathrm{GeV}$ Au-Au collisions with another set of centrality intervals. The symbols represent the STAR data [35]. 


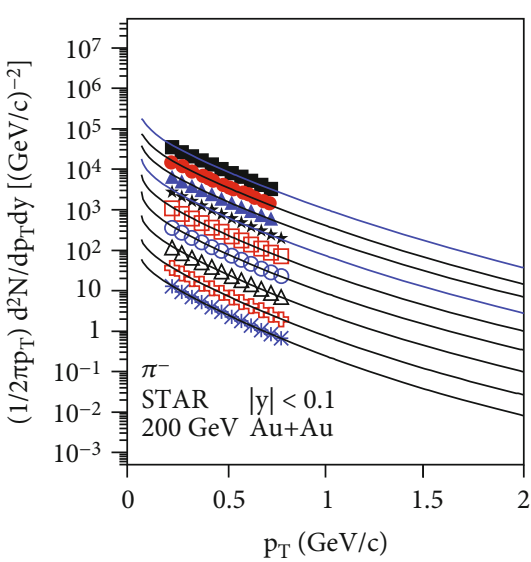

- $0-5 \%(\times 128)$ - $5-10 \%(\times 64)$

^ $10-20 \%(\times 38) \star 20-30 \%(\times 22)$

$\square 30-40 \%(\times 12) \quad \circ 40-50 \%(\times 6)$

$\triangle 50-60 \%(\times 3)$ њ $60-70 \%(\times 1.5)$

米 $70-80 \%$

(a)

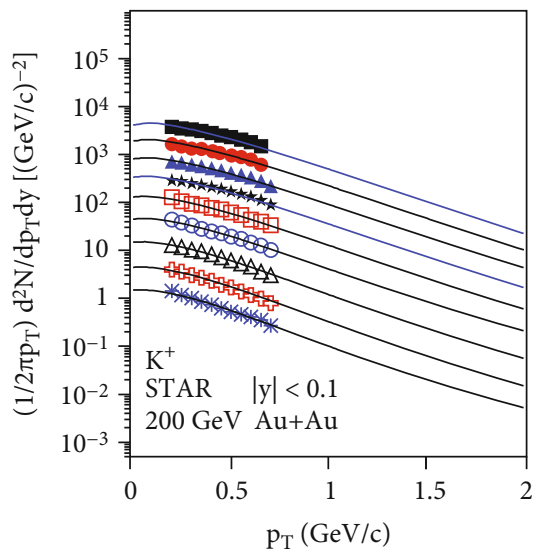

- $0-5 \%(\times 128)$ - $5-10 \%(\times 64)$

^ $10-20 \%(\times 38) \star 20-30 \%(\times 22)$

$30-40 \%(\times 12) \quad \bigcirc 40-50 \%(\times 6)$

$\triangle 50-60 \%(\times 3)$ ↔ $60-70 \%(\times 1.5)$

* $70-80 \%$

(d)

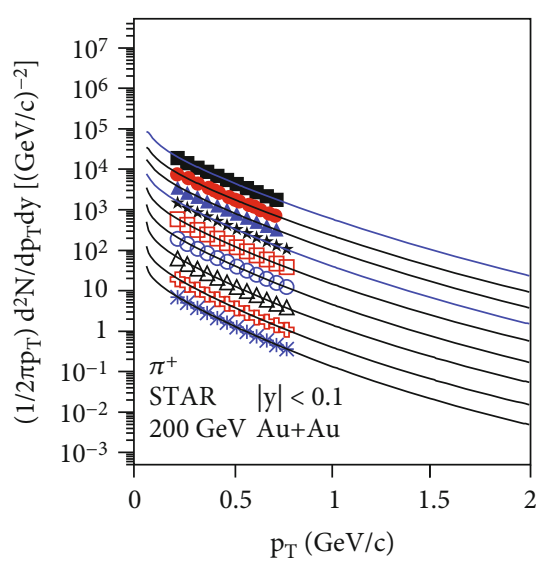

- $0-5 \%(\times 128)$ - $5-10 \%(\times 64)$

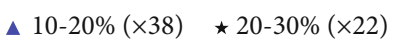

$\square 30-40 \%(\times 12) \quad \circ 40-50 \%(\times 6)$

$\triangle 50-60 \%(\times 3)$ њ $60-70 \%(\times 1.5)$

* $70-80 \%$

(b)

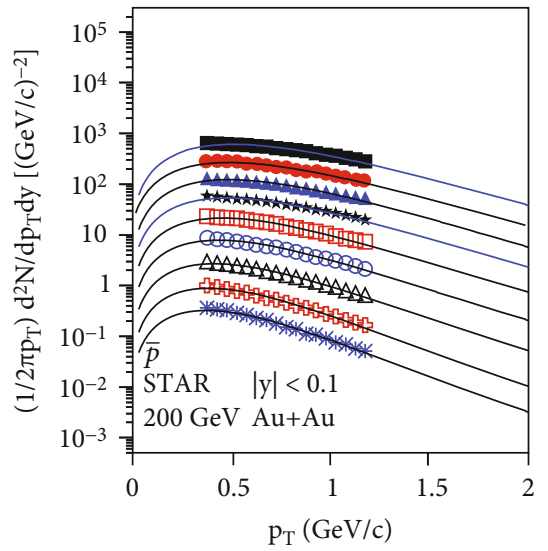

- $0-5 \%(\times 128) \quad 5-10 \%(\times 64)$

^ $10-20 \%(\times 38) \star 20-30 \%(\times 22)$

$\square 30-40 \%(\times 12) \quad \circ 40-50 \%(\times 6)$

$\triangle 50-60 \%(\times 3)$ њ60-70\% $(\times 1.5)$

* $70-80 \%$

(e)

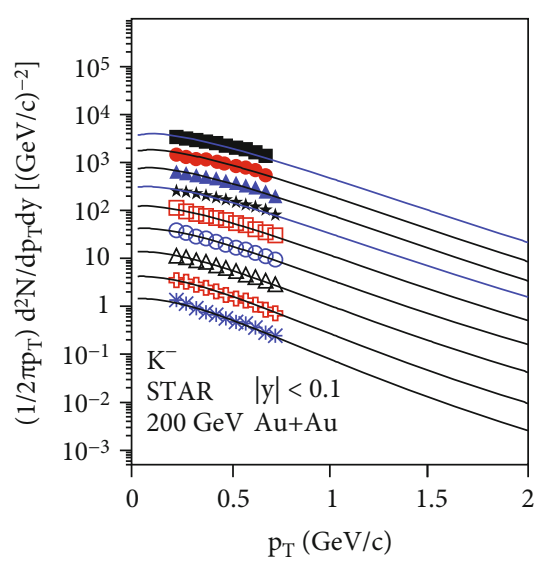

- $0-5 \%(\times 128)$ - 5-10\% (×64)

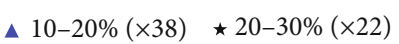

$\square 30-40 \%(\times 12) \quad \circ 40-50 \%(\times 6)$

$\triangle 50-60 \%(\times 3)$ ↔ $60-70 \%(\times 1.5)$

* $70-80 \%$

(c)

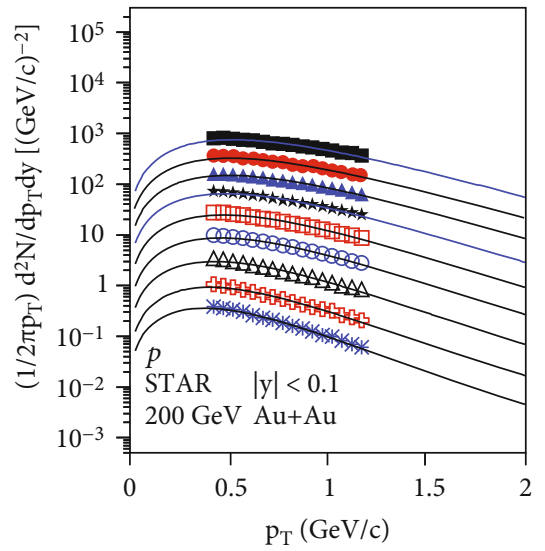

- $0-5 \%(\times 128)$ - $5-10 \%(\times 64)$

^ $10-20 \%(\times 38) \star 20-30 \%(\times 22)$

$\square 30-40 \%(\times 12) \quad \bigcirc 40-50 \%(\times 6)$

$\triangle 50-60 \%(\times 3)$ ↔ $60-70 \%(\times 1.5)$

* $70-80 \%$

(f)

FIgURE 9: Same as Figure 1, but showing the results for $200 \mathrm{GeV}$ Au-Au collisions. The symbols represent the STAR data [35]. 
TABLe 7: Values of $n, T_{0}, a_{0},\left\langle\beta_{t}\right\rangle, \chi^{2}$, and ndof corresponding to the curves in Figure 7.

\begin{tabular}{|c|c|c|c|c|c|c|c|c|}
\hline Figure & Particle & $\sqrt{s_{N N}}(\mathrm{GeV})$ & Selection & $n$ & $T_{0}(\mathrm{GeV})$ & $a_{0}$ & $\left\langle\beta_{t}\right\rangle(c)$ & $\chi^{2} /$ ndof \\
\hline \multirow[t]{9}{*}{ Figure 7(a) } & $\pi^{-}$ & 62.4 & $0-5 \%$ & $8.9 \pm 0.2$ & $0.183 \pm 0.003$ & $-0.419 \pm 0.005$ & $0.125 \pm 0.003$ & $82 / 5$ \\
\hline & & $|y|<0.1$ & $5-10 \%$ & $8.3 \pm 0.2$ & $0.175 \pm 0.003$ & $-0.416 \pm 0.005$ & $0.123 \pm 0.003$ & $83 / 5$ \\
\hline & & & $10-20 \%$ & $7.8 \pm 0.2$ & $0.171 \pm 0.003$ & $-0.414 \pm 0.005$ & $0.122 \pm 0.003$ & $73 / 5$ \\
\hline & & & $20-30 \%$ & $7.5 \pm 0.2$ & $0.168 \pm 0.003$ & $-0.412 \pm 0.005$ & $0.121 \pm 0.003$ & $69 / 5$ \\
\hline & & & $30-40 \%$ & $6.7 \pm 0.1$ & $0.162 \pm 0.003$ & $-0.410 \pm 0.005$ & $0.119 \pm 0.003$ & $62 / 5$ \\
\hline & & & $40-50 \%$ & $6.4 \pm 0.1$ & $0.159 \pm 0.003$ & $-0.408 \pm 0.005$ & $0.118 \pm 0.003$ & $29 / 5$ \\
\hline & & & $50-60 \%$ & $6.0 \pm 0.1$ & $0.154 \pm 0.003$ & $-0.404 \pm 0.005$ & $0.115 \pm 0.003$ & $29 / 5$ \\
\hline & & & $60-70 \%$ & $5.7 \pm 0.1$ & $0.146 \pm 0.002$ & $-0.394 \pm 0.005$ & $0.111 \pm 0.003$ & $20 / 5$ \\
\hline & & & $70-80 \%$ & $5.5 \pm 0.1$ & $0.139 \pm 0.002$ & $-0.388 \pm 0.005$ & $0.106 \pm 0.003$ & $4 / 5$ \\
\hline \multirow[t]{9}{*}{ Figure 7(b) } & $\pi^{+}$ & 62.4 & $0-5 \%$ & $8.2 \pm 0.2$ & $0.195 \pm 0.003$ & $-0.426 \pm 0.005$ & $0.130 \pm 0.003$ & $65 / 5$ \\
\hline & & $|y|<0.1$ & $5-10 \%$ & $7.5 \pm 0.2$ & $0.188 \pm 0.003$ & $-0.424 \pm 0.005$ & $0.126 \pm 0.003$ & $103 / 5$ \\
\hline & & & $10-20 \%$ & $6.6 \pm 0.1$ & $0.178 \pm 0.003$ & $-0.422 \pm 0.005$ & $0.125 \pm 0.003$ & $71 / 5$ \\
\hline & & & $20-30 \%$ & $6.3 \pm 0.1$ & $0.172 \pm 0.003$ & $-0.420 \pm 0.005$ & $0.124 \pm 0.003$ & $84 / 5$ \\
\hline & & & $30-40 \%$ & $6.1 \pm 0.1$ & $0.168 \pm 0.003$ & $-0.418 \pm 0.005$ & $0.123 \pm 0.003$ & $52 / 5$ \\
\hline & & & $40-50 \%$ & $6.0 \pm 0.1$ & $0.161 \pm 0.003$ & $-0.414 \pm 0.005$ & $0.121 \pm 0.003$ & $31 / 5$ \\
\hline & & & $50-60 \%$ & $5.8 \pm 0.1$ & $0.154 \pm 0.003$ & $-0.411 \pm 0.005$ & $0.118 \pm 0.003$ & $29 / 5$ \\
\hline & & & $60-70 \%$ & $5.6 \pm 0.1$ & $0.147 \pm 0.002$ & $-0.407 \pm 0.005$ & $0.115 \pm 0.003$ & $20 / 5$ \\
\hline & & & $70-80 \%$ & $5.6 \pm 0.1$ & $0.140 \pm 0.002$ & $-0.405 \pm 0.005$ & $0.113 \pm 0.003$ & $7 / 5$ \\
\hline \multirow[t]{9}{*}{ Figure 7(c) } & $K^{-}$ & 62.4 & $0-5 \%$ & $15.8 \pm 0.6$ & $0.178 \pm 0.003$ & $-0.038 \pm 0.005$ & $0.193 \pm 0.005$ & $21 / 5$ \\
\hline & & $|y|<0.1$ & $5-10 \%$ & $13.1 \pm 0.4$ & $0.173 \pm 0.003$ & $-0.026 \pm 0.004$ & $0.184 \pm 0.005$ & $4 / 5$ \\
\hline & & & $10-20 \%$ & $12.3 \pm 0.3$ & $0.170 \pm 0.003$ & $-0.018 \pm 0.004$ & $0.175 \pm 0.005$ & $8 / 5$ \\
\hline & & & $20-30 \%$ & $11.3 \pm 0.3$ & $0.166 \pm 0.003$ & $-0.009 \pm 0.004$ & $0.166 \pm 0.005$ & $4 / 5$ \\
\hline & & & $30-40 \%$ & $10.2 \pm 0.3$ & $0.157 \pm 0.003$ & $-0.007 \pm 0.004$ & $0.159 \pm 0.005$ & $5 / 5$ \\
\hline & & & $40-50 \%$ & $9.7 \pm 0.2$ & $0.144 \pm 0.002$ & $-0.022 \pm 0.004$ & $0.152 \pm 0.005$ & $12 / 5$ \\
\hline & & & $50-60 \%$ & $9.4 \pm 0.2$ & $0.136 \pm 0.002$ & $-0.015 \pm 0.004$ & $0.143 \pm 0.005$ & $7 / 5$ \\
\hline & & & $60-70 \%$ & $8.5 \pm 0.2$ & $0.127 \pm 0.002$ & $-0.008 \pm 0.004$ & $0.129 \pm 0.005$ & $7 / 5$ \\
\hline & & & $70-80 \%$ & $7.1 \pm 0.2$ & $0.119 \pm 0.002$ & $0.004 \pm 0.004$ & $0.119 \pm 0.005$ & $9 / 5$ \\
\hline \multirow[t]{9}{*}{ Figure $7(d)$} & $K^{+}$ & 62.4 & $0-5 \%$ & $18.1 \pm 0.8$ & $0.180 \pm 0.003$ & $-0.052 \pm 0.005$ & $0.171 \pm 0.005$ & $11 / 5$ \\
\hline & & $|y|<0.1$ & $5-10 \%$ & $14.2 \pm 0.5$ & $0.177 \pm 0.003$ & $-0.039 \pm 0.005$ & $0.163 \pm 0.005$ & $9 / 5$ \\
\hline & & & $10-20 \%$ & $11.1 \pm 0.3$ & $0.171 \pm 0.003$ & $-0.030 \pm 0.005$ & $0.157 \pm 0.005$ & $8 / 5$ \\
\hline & & & $20-30 \%$ & $9.2 \pm 0.2$ & $0.166 \pm 0.003$ & $-0.027 \pm 0.004$ & $0.149 \pm 0.005$ & $5 / 5$ \\
\hline & & & $30-40 \%$ & $7.4 \pm 0.2$ & $0.158 \pm 0.003$ & $-0.022 \pm 0.004$ & $0.142 \pm 0.005$ & $2 / 5$ \\
\hline & & & $40-50 \%$ & $7.3 \pm 0.2$ & $0.148 \pm 0.002$ & $-0.017 \pm 0.004$ & $0.137 \pm 0.005$ & $4 / 5$ \\
\hline & & & $50-60 \%$ & $6.7 \pm 0.1$ & $0.140 \pm 0.002$ & $-0.016 \pm 0.004$ & $0.132 \pm 0.005$ & $9 / 5$ \\
\hline & & & $60-70 \%$ & $5.9 \pm 0.1$ & $0.135 \pm 0.002$ & $-0.016 \pm 0.004$ & $0.129 \pm 0.005$ & $3 / 5$ \\
\hline & & & $70-80 \%$ & $5.4 \pm 0.1$ & $0.123 \pm 0.002$ & $-0.012 \pm 0.004$ & $0.125 \pm 0.005$ & $8 / 5$ \\
\hline
\end{tabular}


TABle 7: Continued.

\begin{tabular}{|c|c|c|c|c|c|c|c|c|}
\hline Figure & Particle & $\sqrt{s_{N N}}(\mathrm{GeV})$ & Selection & $n$ & $T_{0}(\mathrm{GeV})$ & $a_{0}$ & $\left\langle\beta_{t}\right\rangle(c)$ & $\chi^{2} /$ ndof \\
\hline \multirow[t]{9}{*}{ Figure $7(\mathrm{e})$} & $\bar{p}$ & 62.4 & $0-5 \%$ & $12.6 \pm 0.3$ & $0.142 \pm 0.002$ & $-0.004 \pm 0.004$ & $0.428 \pm 0.008$ & $94 / 11$ \\
\hline & & $|y|<0.1$ & $5-10 \%$ & $12.3 \pm 0.3$ & $0.138 \pm 0.002$ & $0.001 \pm 0.004$ & $0.424 \pm 0.008$ & $105 / 11$ \\
\hline & & & $10-20 \%$ & $11.4 \pm 0.3$ & $0.133 \pm 0.002$ & $0.003 \pm 0.004$ & $0.410 \pm 0.008$ & $81 / 11$ \\
\hline & & & $20-30 \%$ & $10.0 \pm 0.3$ & $0.129 \pm 0.002$ & $0.003 \pm 0.004$ & $0.392 \pm 0.008$ & $114 / 11$ \\
\hline & & & $30-40 \%$ & $10.7 \pm 0.3$ & $0.121 \pm 0.002$ & $0.007 \pm 0.004$ & $0.375 \pm 0.008$ & $96 / 11$ \\
\hline & & & $40-50 \%$ & $9.2 \pm 0.2$ & $0.112 \pm 0.002$ & $0.009 \pm 0.004$ & $0.349 \pm 0.008$ & $76 / 11$ \\
\hline & & & $50-60 \%$ & $8.9 \pm 0.2$ & $0.106 \pm 0.002$ & $0.011 \pm 0.004$ & $0.320 \pm 0.008$ & $50 / 11$ \\
\hline & & & $60-70 \%$ & $8.1 \pm 0.2$ & $0.096 \pm 0.002$ & $0.014 \pm 0.004$ & $0.317 \pm 0.008$ & $75 / 11$ \\
\hline & & & $70-80 \%$ & $7.8 \pm 0.2$ & $0.090 \pm 0.002$ & $0.015 \pm 0.004$ & $0.303 \pm 0.008$ & $99 / 11$ \\
\hline \multirow[t]{9}{*}{ Figure $7(f)$} & $p$ & 62.4 & $0-5 \%$ & $12.7 \pm 0.3$ & $0.148 \pm 0.002$ & $-0.009 \pm 0.004$ & $0.395 \pm 0.008$ & $29 / 10$ \\
\hline & & $|y|<0.1$ & $5-10 \%$ & $12.5 \pm 0.3$ & $0.148 \pm 0.002$ & $-0.005 \pm 0.004$ & $0.390 \pm 0.008$ & $14 / 10$ \\
\hline & & & $10-20 \%$ & $10.8 \pm 0.3$ & $0.142 \pm 0.002$ & $-0.001 \pm 0.004$ & $0.381 \pm 0.008$ & $20 / 10$ \\
\hline & & & $20-30 \%$ & $11.0 \pm 0.3$ & $0.138 \pm 0.002$ & $0.004 \pm 0.004$ & $0.372 \pm 0.008$ & $18 / 10$ \\
\hline & & & $30-40 \%$ & $11.1 \pm 0.3$ & $0.136 \pm 0.002$ & $0.005 \pm 0.004$ & $0.363 \pm 0.008$ & $10 / 10$ \\
\hline & & & $40-50 \%$ & $10.2 \pm 0.3$ & $0.129 \pm 0.002$ & $0.008 \pm 0.004$ & $0.350 \pm 0.008$ & $12 / 10$ \\
\hline & & & $50-60 \%$ & $9.9 \pm 0.2$ & $0.117 \pm 0.002$ & $0.009 \pm 0.004$ & $0.344 \pm 0.008$ & $9 / 10$ \\
\hline & & & $60-70 \%$ & $9.2 \pm 0.2$ & $0.108 \pm 0.002$ & $0.010 \pm 0.004$ & $0.321 \pm 0.008$ & $9 / 10$ \\
\hline & & & $70-80 \%$ & $8.2 \pm 0.2$ & $0.101 \pm 0.002$ & $0.011 \pm 0.004$ & $0.297 \pm 0.007$ & $25 / 10$ \\
\hline
\end{tabular}

TABLE 8: Values of $n, T_{0}, a_{0},\left\langle\beta_{t}\right\rangle, \chi^{2}$, and ndof corresponding to the curves in Figure 8.

\begin{tabular}{|c|c|c|c|c|c|c|c|c|}
\hline Figure & Particle & $\sqrt{s_{N N}}(\mathrm{GeV})$ & Selection & $n$ & $T_{0}(\mathrm{GeV})$ & $a_{0}$ & $\left\langle\beta_{t}\right\rangle(c)$ & $\chi^{2} /$ ndof \\
\hline \multirow[t]{8}{*}{ Figure 8(a) } & $\pi^{-}$ & 130 & $0-6 \%$ & $7.2 \pm 0.2$ & $0.185 \pm 0.003$ & $-0.435 \pm 0.005$ & $0.131 \pm 0.003$ & $54 / 5$ \\
\hline & & $|y|<0.1$ & $6-11 \%$ & $6.8 \pm 0.1$ & $0.177 \pm 0.003$ & $-0.432 \pm 0.005$ & $0.124 \pm 0.003$ & $46 / 5$ \\
\hline & & & $11-18 \%$ & $6.6 \pm 0.1$ & $0.173 \pm 0.003$ & $-0.431 \pm 0.005$ & $0.122 \pm 0.003$ & $34 / 5$ \\
\hline & & & $18-26 \%$ & $6.4 \pm 0.1$ & $0.170 \pm 0.003$ & $-0.430 \pm 0.005$ & $0.121 \pm 0.003$ & $17 / 5$ \\
\hline & & & $26-34 \%$ & $6.2 \pm 0.1$ & $0.165 \pm 0.003$ & $-0.427 \pm 0.005$ & $0.119 \pm 0.003$ & $12 / 5$ \\
\hline & & & $34-45 \%$ & $6.1 \pm 0.1$ & $0.163 \pm 0.003$ & $-0.426 \pm 0.005$ & $0.118 \pm 0.003$ & $14 / 5$ \\
\hline & & & $45-58 \%$ & $5.5 \pm 0.1$ & $0.157 \pm 0.003$ & $-0.424 \pm 0.005$ & $0.115 \pm 0.003$ & $9 / 5$ \\
\hline & & & $58-85 \%$ & $5.3 \pm 0.1$ & $0.150 \pm 0.003$ & $-0.414 \pm 0.005$ & $0.113 \pm 0.003$ & $8 / 5$ \\
\hline \multirow[t]{8}{*}{ Figure 8(b) } & $\pi^{+}$ & 130 & $0-6 \%$ & $8.0 \pm 0.2$ & $0.198 \pm 0.003$ & $-0.429 \pm 0.005$ & $0.137 \pm 0.003$ & $14 / 5$ \\
\hline & & $|y|<0.1$ & $6-11 \%$ & $7.3 \pm 0.2$ & $0.191 \pm 0.003$ & $-0.427 \pm 0.005$ & $0.134 \pm 0.003$ & $13 / 5$ \\
\hline & & & $11-18 \%$ & $6.4 \pm 0.1$ & $0.181 \pm 0.003$ & $-0.424 \pm 0.005$ & $0.132 \pm 0.003$ & $14 / 5$ \\
\hline & & & $18-26 \%$ & $6.1 \pm 0.1$ & $0.177 \pm 0.003$ & $-0.417 \pm 0.005$ & $0.130 \pm 0.003$ & $18 / 5$ \\
\hline & & & $26-34 \%$ & $5.7 \pm 0.1$ & $0.174 \pm 0.003$ & $-0.416 \pm 0.005$ & $0.126 \pm 0.003$ & $13 / 5$ \\
\hline & & & $34-45 \%$ & $5.4 \pm 0.1$ & $0.164 \pm 0.003$ & $-0.413 \pm 0.005$ & $0.125 \pm 0.003$ & $9 / 5$ \\
\hline & & & $45-58 \%$ & $5.4 \pm 0.1$ & $0.159 \pm 0.003$ & $-0.411 \pm 0.005$ & $0.124 \pm 0.003$ & $15 / 5$ \\
\hline & & & $58-85 \%$ & $5.0 \pm 0.1$ & $0.150 \pm 0.003$ & $-0.410 \pm 0.005$ & $0.123 \pm 0.003$ & $18 / 5$ \\
\hline
\end{tabular}


TABle 8: Continued.

\begin{tabular}{|c|c|c|c|c|c|c|c|c|}
\hline Figure & Particle & $\sqrt{s_{N N}}(\mathrm{GeV})$ & Selection & $n$ & $T_{0}(\mathrm{GeV})$ & $a_{0}$ & $\left\langle\beta_{t}\right\rangle(c)$ & $\chi^{2} /$ ndof \\
\hline \multirow[t]{8}{*}{ Figure 8(c) } & $K^{-}$ & 130 & $0-6 \%$ & $14.3 \pm 0.5$ & $0.183 \pm 0.003$ & $-0.038 \pm 0.005$ & $0.209 \pm 0.005$ & $7 / 8$ \\
\hline & & $|y|<0.1$ & $6-11 \%$ & $12.9 \pm 0.3$ & $0.177 \pm 0.003$ & $-0.027 \pm 0.004$ & $0.196 \pm 0.005$ & $9 / 8$ \\
\hline & & & $11-18 \%$ & $10.8 \pm 0.3$ & $0.174 \pm 0.003$ & $-0.019 \pm 0.004$ & $0.181 \pm 0.005$ & $5 / 8$ \\
\hline & & & $18-26 \%$ & $8.3 \pm 0.2$ & $0.168 \pm 0.003$ & $-0.016 \pm 0.004$ & $0.167 \pm 0.005$ & $12 / 8$ \\
\hline & & & $26-34 \%$ & $8.0 \pm 0.2$ & $0.161 \pm 0.003$ & $-0.014 \pm 0.004$ & $0.159 \pm 0.005$ & $7 / 8$ \\
\hline & & & $34-45 \%$ & $7.6 \pm 0.2$ & $0.148 \pm 0.002$ & $-0.007 \pm 0.004$ & $0.154 \pm 0.005$ & $10 / 8$ \\
\hline & & & $45-58 \%$ & $7.1 \pm 0.2$ & $0.141 \pm 0.002$ & $-0.003 \pm 0.004$ & $0.144 \pm 0.005$ & $14 / 8$ \\
\hline & & & $58-85 \%$ & $5.6 \pm 0.1$ & $0.132 \pm 0.002$ & $0.001 \pm 0.004$ & $0.137 \pm 0.005$ & $7 / 8$ \\
\hline \multirow[t]{8}{*}{ Figure $8(\mathrm{~d})$} & $K^{+}$ & 130 & $0-6 \%$ & $15.9 \pm 0.6$ & $0.186 \pm 0.003$ & $-0.054 \pm 0.005$ & $0.194 \pm 0.005$ & $10 / 8$ \\
\hline & & $|y|<0.1$ & $6-11 \%$ & $14.1 \pm 0.5$ & $0.181 \pm 0.003$ & $-0.041 \pm 0.005$ & $0.188 \pm 0.005$ & $10 / 8$ \\
\hline & & & $11-18 \%$ & $11.2 \pm 0.3$ & $0.175 \pm 0.003$ & $-0.032 \pm 0.005$ & $0.182 \pm 0.005$ & $7 / 8$ \\
\hline & & & $18-26 \%$ & $9.3 \pm 0.2$ & $0.170 \pm 0.003$ & $-0.022 \pm 0.004$ & $0.171 \pm 0.005$ & $12 / 8$ \\
\hline & & & $26-34 \%$ & $7.6 \pm 0.2$ & $0.163 \pm 0.003$ & $-0.017 \pm 0.004$ & $0.167 \pm 0.005$ & $17 / 8$ \\
\hline & & & $34-45 \%$ & $6.9 \pm 0.1$ & $0.152 \pm 0.003$ & $-0.013 \pm 0.004$ & $0.162 \pm 0.005$ & $15 / 8$ \\
\hline & & & $45-58 \%$ & $6.3 \pm 0.1$ & $0.144 \pm 0.002$ & $-0.011 \pm 0.004$ & $0.159 \pm 0.005$ & $7 / 8$ \\
\hline & & & $58-85 \%$ & $5.9 \pm 0.1$ & $0.138 \pm 0.002$ & $-0.009 \pm 0.004$ & $0.147 \pm 0.005$ & $15 / 8$ \\
\hline \multirow[t]{8}{*}{ Figure 8(e) } & $\bar{p}$ & 130 & $0-6 \%$ & $12.1 \pm 0.3$ & $0.146 \pm 0.002$ & $-0.006 \pm 0.004$ & $0.457 \pm 0.008$ & $81 / 8$ \\
\hline & & $|y|<0.1$ & $6-11 \%$ & $11.9 \pm 0.3$ & $0.143 \pm 0.002$ & $-0.002 \pm 0.004$ & $0.444 \pm 0.008$ & $33 / 8$ \\
\hline & & & $11-18 \%$ & $11.3 \pm 0.3$ & $0.137 \pm 0.002$ & $0.002 \pm 0.004$ & $0.429 \pm 0.008$ & $12 / 8$ \\
\hline & & & $18-26 \%$ & $10.7 \pm 0.3$ & $0.133 \pm 0.002$ & $0.003 \pm 0.004$ & $0.421 \pm 0.008$ & $32 / 8$ \\
\hline & & & $26-34 \%$ & $9.3 \pm 0.2$ & $0.125 \pm 0.002$ & $0.007 \pm 0.004$ & $0.398 \pm 0.008$ & $25 / 8$ \\
\hline & & & $34-45 \%$ & $9.0 \pm 0.2$ & $0.117 \pm 0.002$ & $0.008 \pm 0.004$ & $0.375 \pm 0.008$ & $26 / 8$ \\
\hline & & & $45-58 \%$ & $8.7 \pm 0.2$ & $0.110 \pm 0.002$ & $0.011 \pm 0.004$ & $0.367 \pm 0.008$ & $25 / 8$ \\
\hline & & & $58-85 \%$ & $7.9 \pm 0.2$ & $0.102 \pm 0.002$ & $0.012 \pm 0.004$ & $0.328 \pm 0.008$ & $22 / 8$ \\
\hline \multirow[t]{8}{*}{ Figure $8(\mathrm{f})$} & $p$ & 130 & $0-6 \%$ & $12.3 \pm 0.4$ & $0.149 \pm 0.002$ & $-0.009 \pm 0.004$ & $0.439 \pm 0.008$ & $66 / 8$ \\
\hline & & $|y|<0.1$ & $6-11 \%$ & $12.0 \pm 0.4$ & $0.148 \pm 0.002$ & $-0.006 \pm 0.004$ & $0.432 \pm 0.008$ & $41 / 8$ \\
\hline & & & $11-18 \%$ & $10.6 \pm 0.3$ & $0.144 \pm 0.002$ & $-0.002 \pm 0.004$ & $0.425 \pm 0.008$ & $13 / 8$ \\
\hline & & & $18-26 \%$ & $9.2 \pm 0.2$ & $0.139 \pm 0.002$ & $0.001 \pm 0.004$ & $0.411 \pm 0.008$ & $6 / 8$ \\
\hline & & & $26-34 \%$ & $8.9 \pm 0.2$ & $0.133 \pm 0.002$ & $0.004 \pm 0.004$ & $0.398 \pm 0.008$ & $16 / 8$ \\
\hline & & & $34-45 \%$ & $8.1 \pm 0.2$ & $0.129 \pm 0.002$ & $0.007 \pm 0.004$ & $0.375 \pm 0.008$ & $24 / 8$ \\
\hline & & & $45-58 \%$ & $7.7 \pm 0.2$ & $0.117 \pm 0.002$ & $0.008 \pm 0.004$ & $0.363 \pm 0.008$ & $10 / 8$ \\
\hline & & & $58-85 \%$ & $7.4 \pm 0.2$ & $0.102 \pm 0.002$ & $0.010 \pm 0.004$ & $0.333 \pm 0.008$ & $11 / 8$ \\
\hline
\end{tabular}


TABLE 9: Values of $n, T_{0}, a_{0},\left\langle\beta_{t}\right\rangle, \chi^{2}$, and ndof corresponding to the curves in Figure 9.

\begin{tabular}{|c|c|c|c|c|c|c|c|c|}
\hline Figure & Particle & $\sqrt{s_{N N}}(\mathrm{GeV})$ & Selection & $n$ & $T_{0}(\mathrm{GeV})$ & $a_{0}$ & $\left\langle\beta_{t}\right\rangle(c)$ & $\chi^{2} /$ ndof \\
\hline \multirow[t]{9}{*}{ Figure 9(a) } & $\pi^{-}$ & 200 & $0-5 \%$ & $6.7 \pm 0.1$ & $0.189 \pm 0.003$ & $-0.439 \pm 0.005$ & $0.139 \pm 0.003$ & $58 / 6$ \\
\hline & & $|y|<0.1$ & $5-10 \%$ & $6.1 \pm 0.1$ & $0.180 \pm 0.003$ & $-0.437 \pm 0.005$ & $0.138 \pm 0.003$ & $50 / 6$ \\
\hline & & & $10-20 \%$ & $5.7 \pm 0.1$ & $0.178 \pm 0.003$ & $-0.434 \pm 0.005$ & $0.135 \pm 0.003$ & $77 / 6$ \\
\hline & & & $20-30 \%$ & $5.5 \pm 0.1$ & $0.174 \pm 0.003$ & $-0.432 \pm 0.005$ & $0.133 \pm 0.003$ & $64 / 7$ \\
\hline & & & $30-40 \%$ & $5.2 \pm 0.1$ & $0.168 \pm 0.003$ & $-0.430 \pm 0.005$ & $0.132 \pm 0.003$ & $54 / 7$ \\
\hline & & & $40-50 \%$ & $5.1 \pm 0.1$ & $0.167 \pm 0.003$ & $-0.426 \pm 0.005$ & $0.125 \pm 0.003$ & $46 / 7$ \\
\hline & & & $50-60 \%$ & $5.0 \pm 0.1$ & $0.160 \pm 0.003$ & $-0.423 \pm 0.005$ & $0.123 \pm 0.003$ & $33 / 7$ \\
\hline & & & $60-70 \%$ & $5.0 \pm 0.1$ & $0.153 \pm 0.003$ & $-0.420 \pm 0.005$ & $0.120 \pm 0.003$ & $13 / 7$ \\
\hline & & & $70-80 \%$ & $4.9 \pm 0.1$ & $0.145 \pm 0.002$ & $-0.419 \pm 0.005$ & $0.116 \pm 0.003$ & $5 / 7$ \\
\hline \multirow[t]{9}{*}{ Figure 9(b) } & $\pi^{+}$ & 200 & $0-5 \%$ & $7.0 \pm 0.1$ & $0.201 \pm 0.003$ & $-0.432 \pm 0.005$ & $0.141 \pm 0.003$ & $29 / 6$ \\
\hline & & $|y|<0.1$ & $5-10 \%$ & $6.4 \pm 0.1$ & $0.194 \pm 0.003$ & $-0.426 \pm 0.005$ & $0.139 \pm 0.003$ & $24 / 6$ \\
\hline & & & $10-20 \%$ & $6.3 \pm 0.1$ & $0.184 \pm 0.003$ & $-0.421 \pm 0.005$ & $0.137 \pm 0.003$ & $42 / 6$ \\
\hline & & & $20-30 \%$ & $6.1 \pm 0.1$ & $0.180 \pm 0.003$ & $-0.418 \pm 0.005$ & $0.135 \pm 0.003$ & $25 / 7$ \\
\hline & & & $30-40 \%$ & $5.7 \pm 0.1$ & $0.177 \pm 0.003$ & $-0.416 \pm 0.005$ & $0.132 \pm 0.003$ & $16 / 7$ \\
\hline & & & $40-50 \%$ & $5.5 \pm 0.1$ & $0.167 \pm 0.003$ & $-0.412 \pm 0.005$ & $0.131 \pm 0.003$ & $31 / 7$ \\
\hline & & & $50-60 \%$ & $5.2 \pm 0.1$ & $0.162 \pm 0.003$ & $-0.406 \pm 0.005$ & $0.130 \pm 0.003$ & $26 / 7$ \\
\hline & & & $60-70 \%$ & $4.9 \pm 0.1$ & $0.153 \pm 0.003$ & $-0.403 \pm 0.005$ & $0.125 \pm 0.003$ & $11 / 7$ \\
\hline & & & $70-80 \%$ & $4.8 \pm 0.1$ & $0.146 \pm 0.002$ & $-0.401 \pm 0.005$ & $0.124 \pm 0.003$ & $2 / 7$ \\
\hline \multirow[t]{9}{*}{ Figure 9(c) } & $K^{-}$ & 200 & $0-5 \%$ & $12.4 \pm 0.3$ & $0.187 \pm 0.003$ & $-0.040 \pm 0.005$ & $0.232 \pm 0.005$ & $9 / 5$ \\
\hline & & $|y|<0.1$ & $5-10 \%$ & $11.3 \pm 0.3$ & $0.181 \pm 0.003$ & $-0.033 \pm 0.005$ & $0.212 \pm 0.005$ & $8 / 5$ \\
\hline & & & $10-20 \%$ & $10.4 \pm 0.3$ & $0.178 \pm 0.003$ & $-0.026 \pm 0.004$ & $0.195 \pm 0.005$ & $8 / 6$ \\
\hline & & & $20-30 \%$ & $7.9 \pm 0.2$ & $0.174 \pm 0.003$ & $-0.018 \pm 0.004$ & $0.183 \pm 0.005$ & $8 / 6$ \\
\hline & & & $30-40 \%$ & $7.4 \pm 0.2$ & $0.165 \pm 0.003$ & $-0.015 \pm 0.004$ & $0.173 \pm 0.005$ & $5 / 6$ \\
\hline & & & $40-50 \%$ & $6.0 \pm 0.1$ & $0.152 \pm 0.003$ & $-0.010 \pm 0.004$ & $0.162 \pm 0.005$ & $5 / 6$ \\
\hline & & & $50-60 \%$ & $5.9 \pm 0.1$ & $0.145 \pm 0.002$ & $-0.007 \pm 0.004$ & $0.150 \pm 0.005$ & $4 / 6$ \\
\hline & & & $60-70 \%$ & $5.9 \pm 0.1$ & $0.136 \pm 0.002$ & $-0.004 \pm 0.004$ & $0.136 \pm 0.005$ & $4 / 6$ \\
\hline & & & $70-80 \%$ & $5.6 \pm 0.1$ & $0.127 \pm 0.002$ & $-0.003 \pm 0.004$ & $0.128 \pm 0.005$ & $12 / 6$ \\
\hline \multirow[t]{9}{*}{ Figure $9(d)$} & $K^{+}$ & 200 & $0-5 \%$ & $13.9 \pm 0.3$ & $0.188 \pm 0.003$ & $-0.053 \pm 0.005$ & $0.239 \pm 0.005$ & $10 / 5$ \\
\hline & & $|y|<0.1$ & $5-10 \%$ & $11.6 \pm 0.3$ & $0.183 \pm 0.003$ & $-0.039 \pm 0.005$ & $0.227 \pm 0.005$ & $8 / 5$ \\
\hline & & & $10-20 \%$ & $9.6 \pm 0.2$ & $0.178 \pm 0.003$ & $-0.030 \pm 0.005$ & $0.217 \pm 0.005$ & $6 / 6$ \\
\hline & & & $20-30 \%$ & $8.7 \pm 0.2$ & $0.173 \pm 0.003$ & $-0.026 \pm 0.004$ & $0.208 \pm 0.005$ & $5 / 6$ \\
\hline & & & $30-40 \%$ & $7.7 \pm 0.2$ & $0.166 \pm 0.003$ & $-0.020 \pm 0.004$ & $0.197 \pm 0.005$ & $4 / 6$ \\
\hline & & & $40-50 \%$ & $6.0 \pm 0.1$ & $0.156 \pm 0.003$ & $-0.017 \pm 0.004$ & $0.187 \pm 0.005$ & $4 / 6$ \\
\hline & & & $50-60 \%$ & $5.8 \pm 0.1$ & $0.148 \pm 0.002$ & $-0.014 \pm 0.004$ & $0.176 \pm 0.005$ & $3 / 6$ \\
\hline & & & $60-70 \%$ & $5.5 \pm 0.1$ & $0.143 \pm 0.002$ & $-0.012 \pm 0.004$ & $0.162 \pm 0.005$ & $5 / 6$ \\
\hline & & & $70-80 \%$ & $4.4 \pm 0.1$ & $0.131 \pm 0.002$ & $-0.007 \pm 0.004$ & $0.155 \pm 0.005$ & $6 / 6$ \\
\hline \multirow[t]{9}{*}{ Figure 9(e) } & $\bar{p}$ & 200 & $0-5 \%$ & $11.8 \pm 0.3$ & $0.151 \pm 0.003$ & $-0.012 \pm 0.004$ & $0.472 \pm 0.008$ & $79 / 12$ \\
\hline & & $|y|<0.1$ & $5-10 \%$ & $10.5 \pm 0.3$ & $0.147 \pm 0.002$ & $-0.011 \pm 0.004$ & $0.464 \pm 0.008$ & $89 / 12$ \\
\hline & & & $10-20 \%$ & $10.3 \pm 0.3$ & $0.141 \pm 0.002$ & $-0.010 \pm 0.004$ & $0.446 \pm 0.008$ & $80 / 12$ \\
\hline & & & $20-30 \%$ & $8.4 \pm 0.2$ & $0.138 \pm 0.002$ & $-0.010 \pm 0.004$ & $0.428 \pm 0.008$ & $89 / 12$ \\
\hline & & & $30-40 \%$ & $6.8 \pm 0.1$ & $0.129 \pm 0.002$ & $-0.009 \pm 0.004$ & $0.399 \pm 0.008$ & $57 / 12$ \\
\hline & & & $40-50 \%$ & $6.3 \pm 0.1$ & $0.121 \pm 0.002$ & $-0.007 \pm 0.004$ & $0.374 \pm 0.008$ & $74 / 12$ \\
\hline & & & $50-60 \%$ & $6.2 \pm 0.1$ & $0.115 \pm 0.002$ & $-0.005 \pm 0.004$ & $0.347 \pm 0.008$ & $44 / 12$ \\
\hline & & & $60-70 \%$ & $5.9 \pm 0.1$ & $0.104 \pm 0.002$ & $-0.005 \pm 0.004$ & $0.317 \pm 0.008$ & $69 / 12$ \\
\hline & & & $70-80 \%$ & $5.8 \pm 0.1$ & $0.103 \pm 0.002$ & $-0.002 \pm 0.004$ & $0.289 \pm 0.007$ & $43 / 12$ \\
\hline
\end{tabular}


TABle 9: Continued.

\begin{tabular}{|c|c|c|c|c|c|c|c|c|}
\hline Figure & Particle & $\sqrt{s_{N N}}(\mathrm{GeV})$ & Selection & $n$ & $T_{0}(\mathrm{GeV})$ & $a_{0}$ & $\left\langle\beta_{t}\right\rangle(c)$ & $\chi^{2} /$ ndof \\
\hline \multirow[t]{9}{*}{ Figure $9(\mathrm{f})$} & $p$ & 200 & $0-5 \%$ & $11.7 \pm 0.3$ & $0.154 \pm 0.003$ & $-0.011 \pm 0.004$ & $0.492 \pm 0.008$ & $30 / 11$ \\
\hline & & $|y|<0.1$ & $5-10 \%$ & $11.2 \pm 0.3$ & $0.151 \pm 0.003$ & $-0.008 \pm 0.004$ & $0.478 \pm 0.008$ & $35 / 11$ \\
\hline & & & $10-20 \%$ & $10.8 \pm 0.3$ & $0.145 \pm 0.002$ & $-0.005 \pm 0.004$ & $0.466 \pm 0.008$ & $23 / 11$ \\
\hline & & & $20-30 \%$ & $8.8 \pm 0.2$ & $0.133 \pm 0.002$ & $0.002 \pm 0.004$ & $0.449 \pm 0.008$ & $31 / 11$ \\
\hline & & & $30-40 \%$ & $8.1 \pm 0.2$ & $0.127 \pm 0.002$ & $0.003 \pm 0.004$ & $0.436 \pm 0.008$ & $29 / 11$ \\
\hline & & & $40-50 \%$ & $7.5 \pm 0.2$ & $0.122 \pm 0.002$ & $0.004 \pm 0.004$ & $0.417 \pm 0.008$ & $30 / 11$ \\
\hline & & & $50-60 \%$ & $6.8 \pm 0.1$ & $0.118 \pm 0.002$ & $0.006 \pm 0.004$ & $0.371 \pm 0.008$ & $44 / 11$ \\
\hline & & & $60-70 \%$ & $5.3 \pm 0.1$ & $0.107 \pm 0.002$ & $0.008 \pm 0.004$ & $0.338 \pm 0.008$ & $36 / 11$ \\
\hline & & & $70-80 \%$ & $5.0 \pm 0.1$ & $0.100 \pm 0.002$ & $0.010 \pm 0.004$ & $0.300 \pm 0.007$ & $14 / 11$ \\
\hline
\end{tabular}

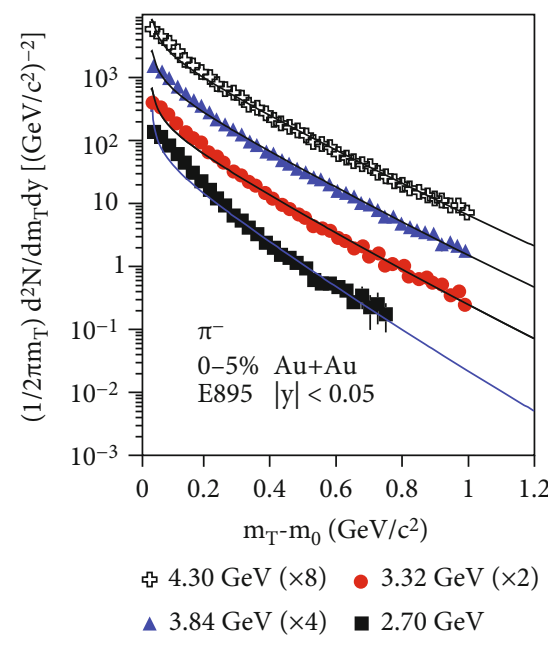

(a)

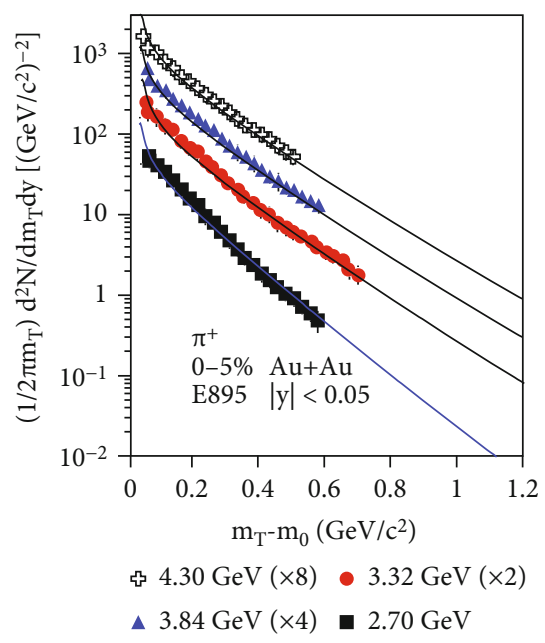

(b)

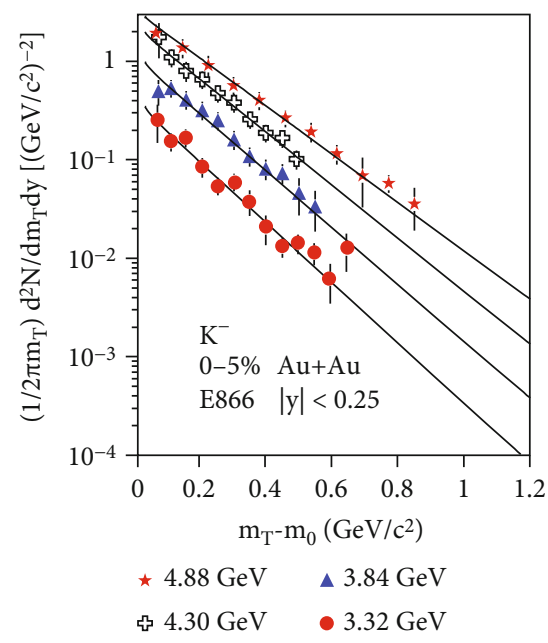

(c)

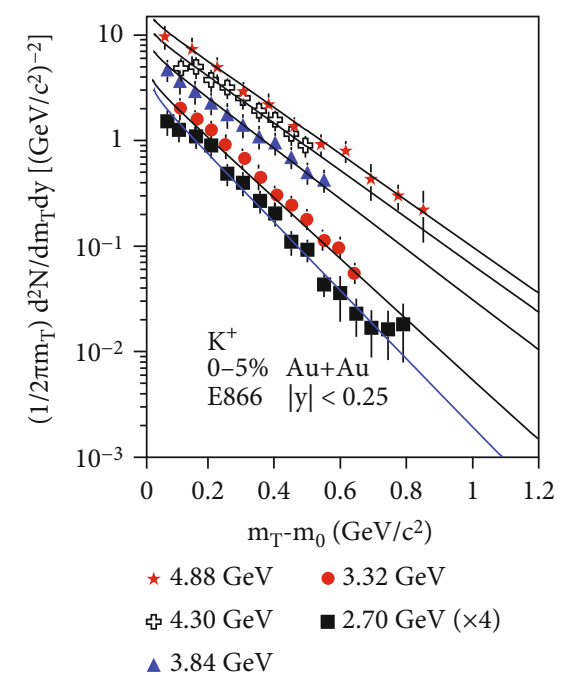

(d)

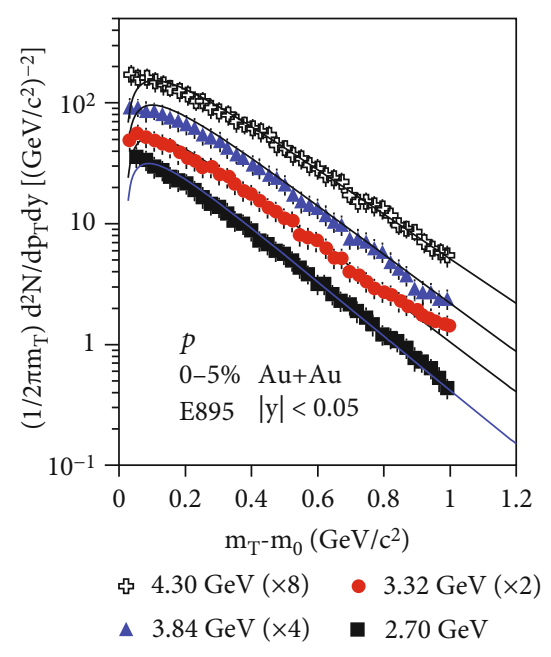

(e)

Figure 10: Transverse mass spectra of $\pi^{-}(\mathrm{a}), \pi^{+}(\mathrm{b}), K^{-}(\mathrm{c}), K^{+}(\mathrm{d})$, and $p$ (e) produced in $0-5 \%$ Au-Au collisions at midrapidity at $\sqrt{s_{N N}}=2.70,3.32,3.84,4.30$, and $4.88 \mathrm{GeV}$, where only panel (d) contains five energies. The symbols in panels (a), (b), and (e) represent the experimental data measured by the E895 Collaboration [36], and those in panels (c) and (d) are from the E866 Collaboration [37], where some sets of data are rescaled by different amounts. The data for $\bar{p}$ is not available in the same or similar experiments. The curves are our results fitted by Equation (4) for $\pi^{\mp}$ and $K^{\mp}$ (panels (a)-(d)) or Equation (6) for $p$ (panel (e)). 
TABLE 10: Values of $n, T_{0}, a_{0},\left\langle\beta_{t}\right\rangle, \chi^{2}$, and ndof corresponding to the curves in Figure 10.

\begin{tabular}{|c|c|c|c|c|c|c|c|c|}
\hline Figure & Particle & $\sqrt{s_{N N}}(\mathrm{GeV})$ & Selection & $n$ & $T_{0}(\mathrm{GeV})$ & $a_{0}$ & $\left\langle\beta_{t}\right\rangle(c)$ & $\chi^{2} /$ ndof \\
\hline \multirow[t]{4}{*}{ Figure 10(a) } & $\pi^{-}$ & 2.70 & $0-5 \%$ & $20.6 \pm 1.0$ & $0.104 \pm 0.002$ & $-0.455 \pm 0.005$ & $0.098 \pm 0.002$ & $35 / 25$ \\
\hline & & 3.32 & $|y|<0.05$ & $19.9 \pm 0.9$ & $0.127 \pm 0.002$ & $-0.441 \pm 0.005$ & $0.105 \pm 0.003$ & $30 / 35$ \\
\hline & & 3.84 & & $19.0 \pm 0.8$ & $0.135 \pm 0.002$ & $-0.430 \pm 0.005$ & $0.107 \pm 0.003$ & $13 / 35$ \\
\hline & & 4.30 & & $18.2 \pm 0.8$ & $0.141 \pm 0.002$ & $-0.424 \pm 0.005$ & $0.110 \pm 0.003$ & $13 / 35$ \\
\hline \multirow[t]{4}{*}{ Figure 10(b) } & $\pi^{+}$ & 2.70 & $0-5 \%$ & $21.3 \pm 1.1$ & $0.107 \pm 0.002$ & $-0.463 \pm 0.005$ & $0.110 \pm 0.003$ & $53 / 18$ \\
\hline & & 3.32 & $|y|<0.05$ & $20.5 \pm 1.0$ & $0.137 \pm 0.002$ & $-0.458 \pm 0.005$ & $0.112 \pm 0.003$ & $56 / 23$ \\
\hline & & 3.84 & & $19.8 \pm 0.9$ & $0.144 \pm 0.002$ & $-0.443 \pm 0.005$ & $0.114 \pm 0.003$ & $54 / 18$ \\
\hline & & 4.30 & & $19.0 \pm 0.8$ & $0.147 \pm 0.002$ & $-0.438 \pm 0.005$ & $0.116 \pm 0.003$ & $61 / 15$ \\
\hline \multirow[t]{4}{*}{ Figure $10(c)$} & $K^{-}$ & 3.32 & $0-5 \%$ & $31.7 \pm 2.1$ & $0.108 \pm 0.002$ & $-0.044 \pm 0.005$ & $0.092 \pm 0.004$ & $9 / 8$ \\
\hline & & 3.84 & $|y|<0.25$ & $30.4 \pm 2.0$ & $0.114 \pm 0.002$ & $-0.037 \pm 0.005$ & $0.095 \pm 0.004$ & $4 / 6$ \\
\hline & & 4.30 & & $29.6 \pm 1.9$ & $0.122 \pm 0.002$ & $-0.028 \pm 0.004$ & $0.099 \pm 0.004$ & $1 / 5$ \\
\hline & & 4.88 & & $28.8 \pm 1.8$ & $0.134 \pm 0.002$ & $-0.020 \pm 0.004$ & $0.103 \pm 0.005$ & $1 / 6$ \\
\hline \multirow[t]{5}{*}{ Figure $10(\mathrm{~d})$} & $K^{+}$ & 2.70 & $0-5 \%$ & $33.5 \pm 2.3$ & $0.102 \pm 0.002$ & $-0.064 \pm 0.005$ & $0.103 \pm 0.005$ & $6 / 11$ \\
\hline & & 3.32 & $|y|<0.25$ & $32.4 \pm 2.2$ & $0.115 \pm 0.002$ & $-0.057 \pm 0.005$ & $0.109 \pm 0.005$ & $1 / 7$ \\
\hline & & 3.84 & & $31.8 \pm 2.1$ & $0.140 \pm 0.002$ & $-0.048 \pm 0.005$ & $0.113 \pm 0.005$ & $1 / 6$ \\
\hline & & 4.30 & & $31.0 \pm 2.0$ & $0.149 \pm 0.002$ & $-0.039 \pm 0.005$ & $0.116 \pm 0.005$ & $1 / 4$ \\
\hline & & 4.88 & & $30.3 \pm 2.0$ & $0.151 \pm 0.002$ & $-0.036 \pm 0.005$ & $0.119 \pm 0.005$ & $1 / 6$ \\
\hline \multirow[t]{4}{*}{ Figure 10(e) } & $p$ & 2.70 & $0-5 \%$ & $22.0 \pm 1.1$ & $0.106 \pm 0.002$ & $-0.016 \pm 0.004$ & $0.324 \pm 0.005$ & $8 / 34$ \\
\hline & & 3.32 & $|y|<0.05$ & $21.3 \pm 1.1$ & $0.114 \pm 0.002$ & $-0.013 \pm 0.004$ & $0.330 \pm 0.005$ & $14 / 35$ \\
\hline & & 3.84 & & $20.5 \pm 1.0$ & $0.116 \pm 0.002$ & $-0.010 \pm 0.004$ & $0.334 \pm 0.005$ & $9 / 35$ \\
\hline & & 4.30 & & $19.6 \pm 0.9$ & $0.122 \pm 0.002$ & $-0.003 \pm 0.004$ & $0.342 \pm 0.005$ & $9 / 35$ \\
\hline
\end{tabular}




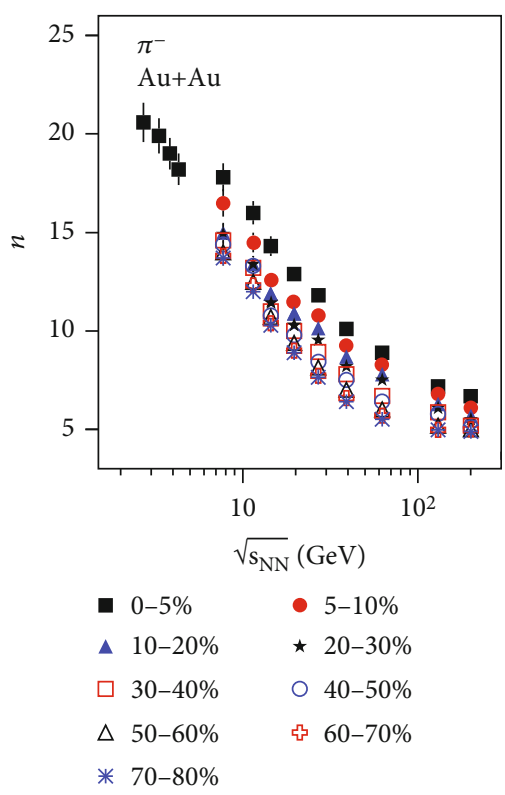

(a)

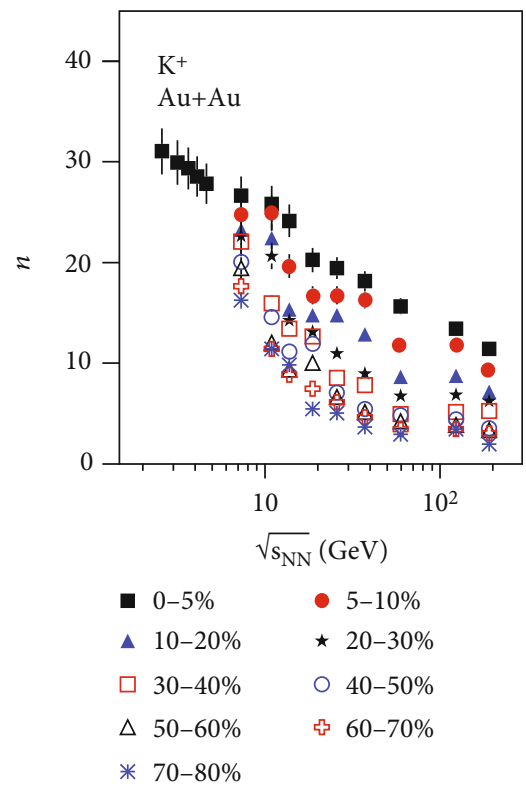

(d)

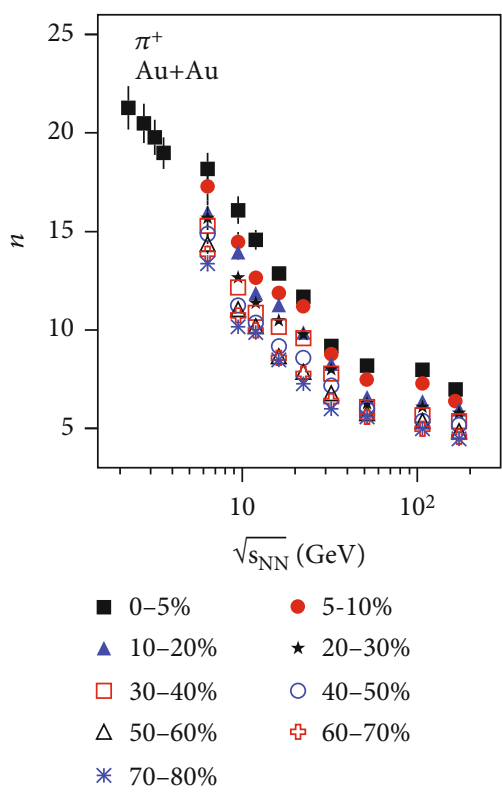

(b)

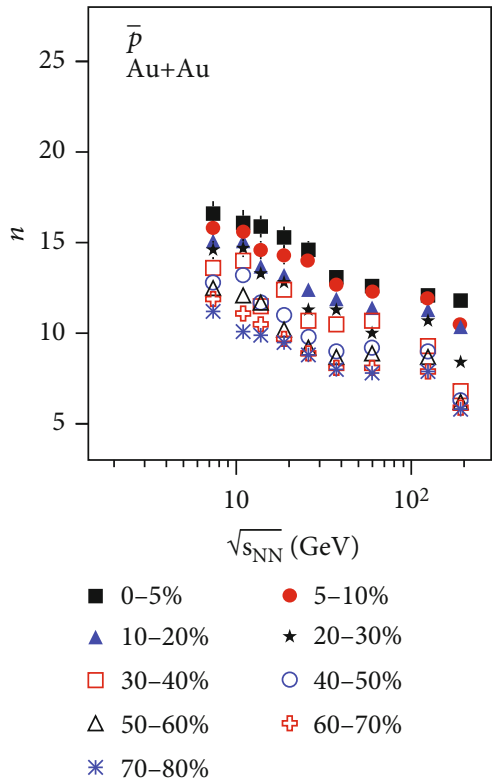

(e)

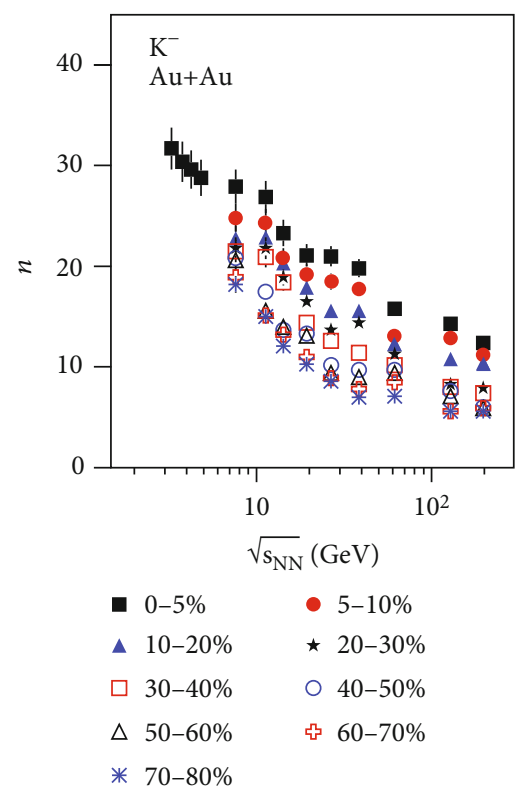

(c)

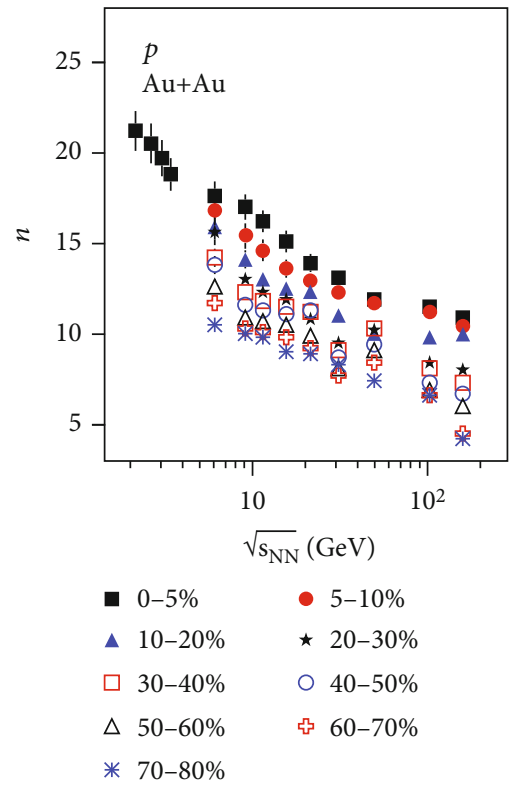

(f)

FIgURE 11: Dependence of power index $n$ on collision energy $\sqrt{s_{N N}}$ in Au-Au collision with nine main centrality intervals which are marked in the panels. (a-f) correspond to the results from $\pi^{-}, \pi^{+}, K^{-}, K^{+}, \bar{p}$, and $p$ spectra, respectively. In particular, at $130 \mathrm{GeV}$, the centrality intervals $0-6 \%, 6-11 \%, 11-18 \%, 18-26 \%, 26-34 \%, 34-45 \%$, and $45-58 \%$ are orderly classified into the closest main centrality intervals, and the centrality interval $58-85 \%$ is simultaneously classified into $60-70 \%$ and $70-80 \%$. The results for different particles are cited in Tables $1-10$ 


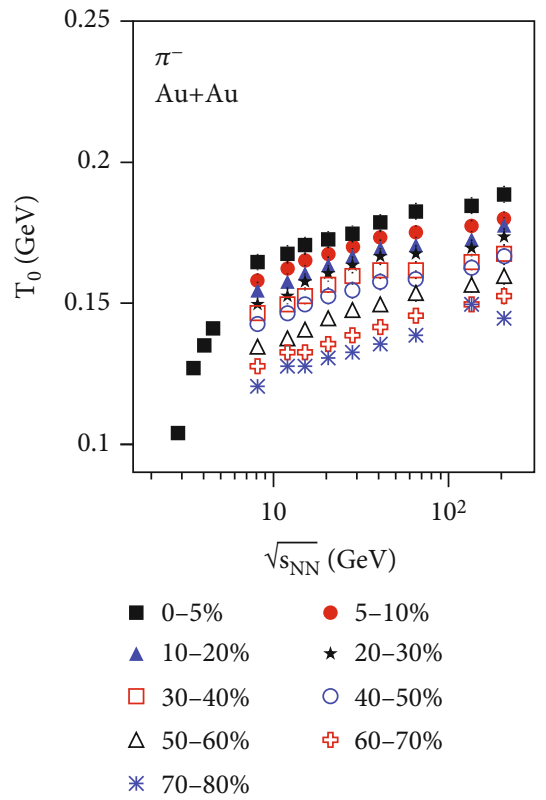

(a)

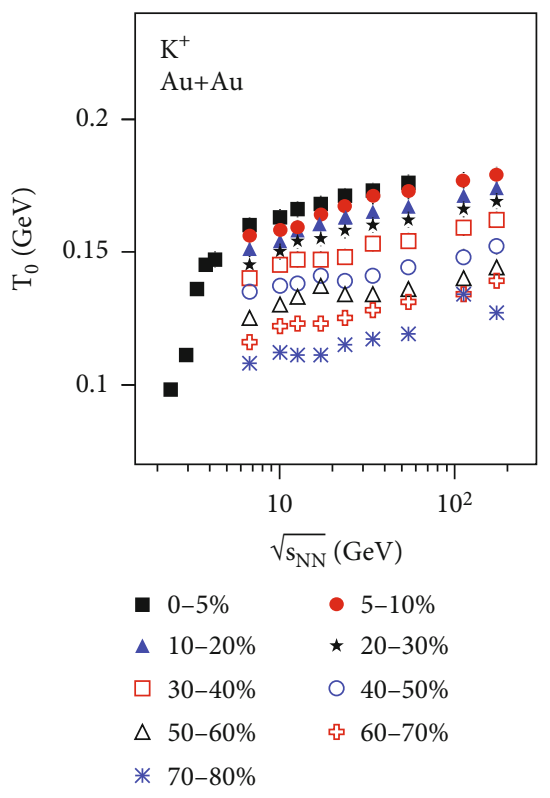

(d)

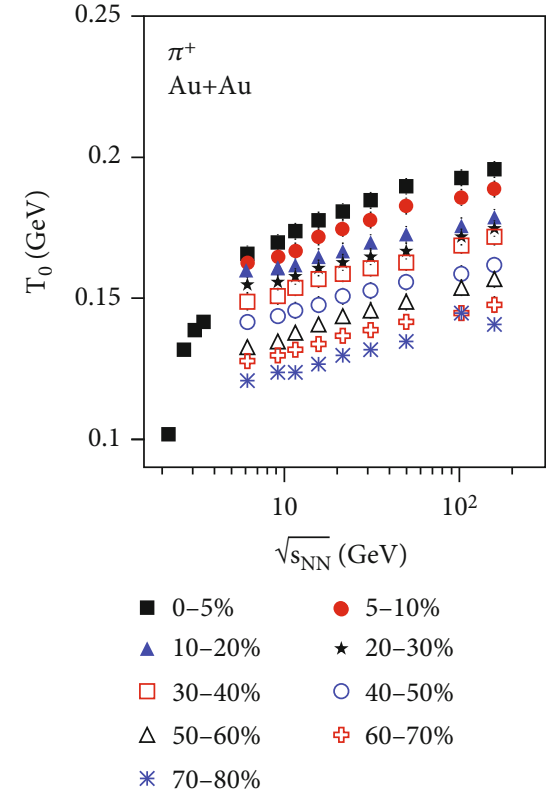

(b)

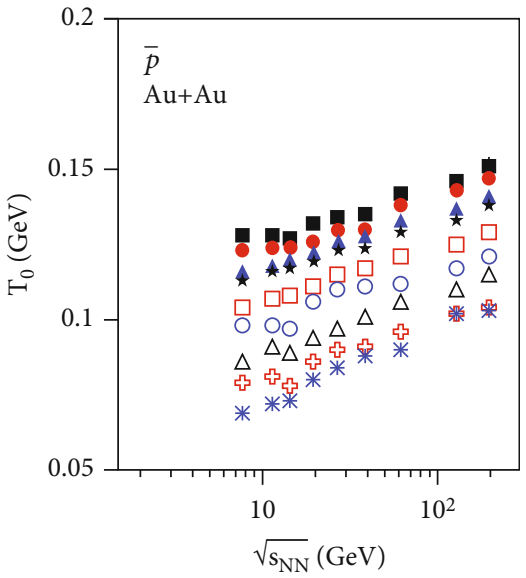

- $0-5 \%$ - $5-10 \%$

- $10-20 \%$

$\star 20-30 \%$

$30-40 \% \quad \circ \quad 40-50 \%$

$\triangle 50-60 \%$ ¡ $60-70 \%$

* $70-80 \%$

(e)

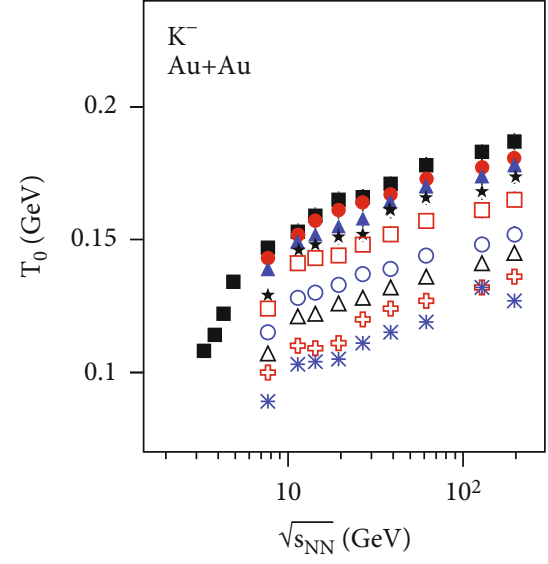

- $0-5 \%$ - $5-10 \%$

$\triangle 10-20 \% \quad \star 20-30 \%$

$\square \quad 30-40 \% \quad \bigcirc \quad 40-50 \%$

$\triangle 50-60 \%$ ↔ $60-70 \%$

* $70-80 \%$

(c)

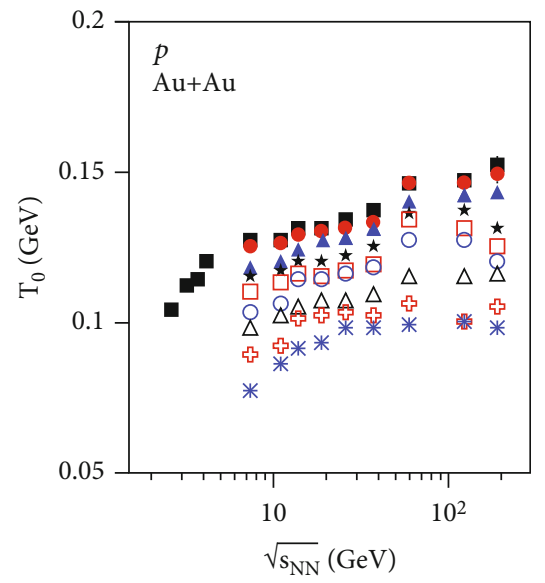
- $0-5 \%$
- $5-10 \%$
\ $10-20 \%$

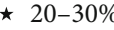
口 $30-40 \%$
○ $40-50 \%$
$\triangle 50-60 \%$
† $60-70 \%$
* $70-80 \%$

(f)

FIgURE 12: Same as Figure 11, but showing the dependence of thermal freeze-out temperature $T_{0}$ on $\sqrt{s_{N N}}$. 


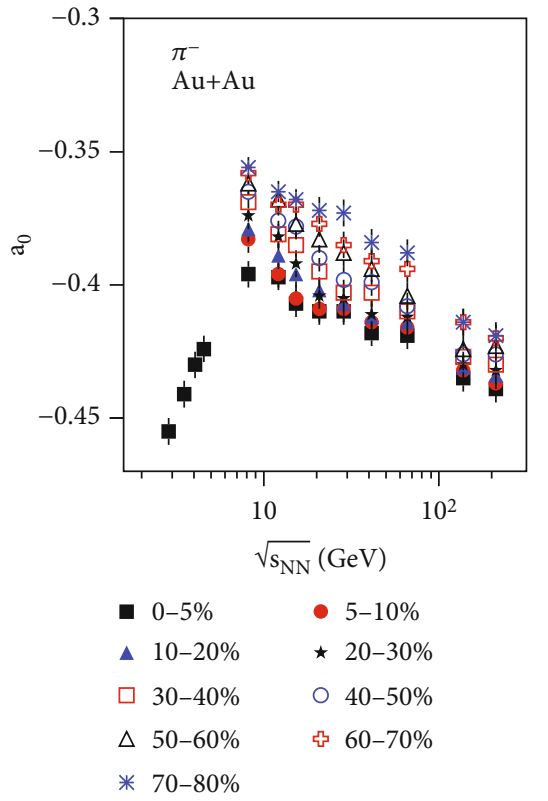

(a)

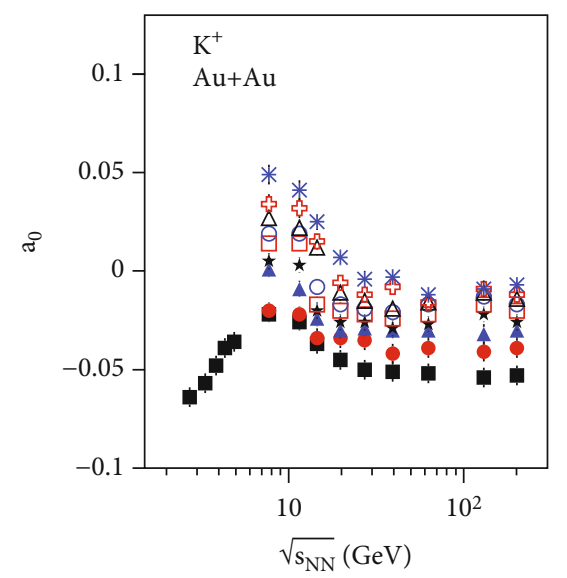

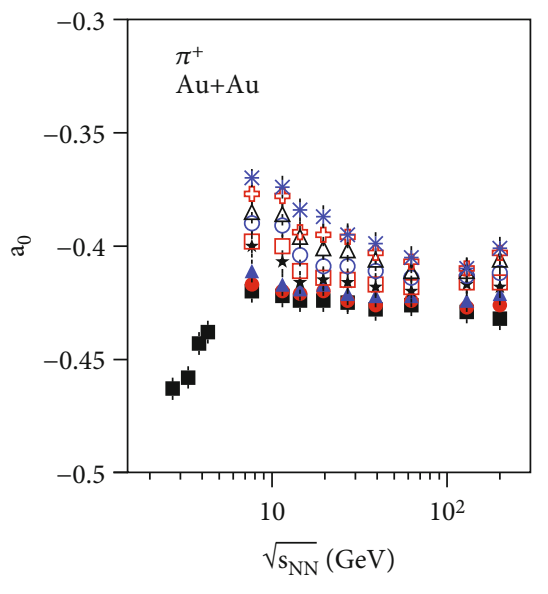

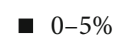

- $5-10 \%$

- $10-20 \%$

^ 20-30\%

$\square \quad 30-40 \%$

○ $40-50 \%$

$\triangle 50-60 \%$

њ $60-70 \%$

* $70-80 \%$

(b)

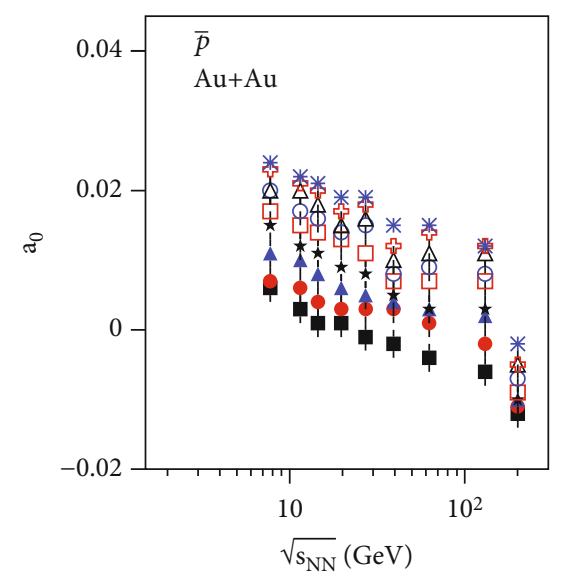

- $0-5 \%$

- $5-10 \%$

- $10-20 \%$

^ 20-30\%

$\square \quad 30-40 \%$

○ $40-50 \%$

$\triangle 50-60 \%$

њ $60-70 \%$

* $70-80 \%$

(e)

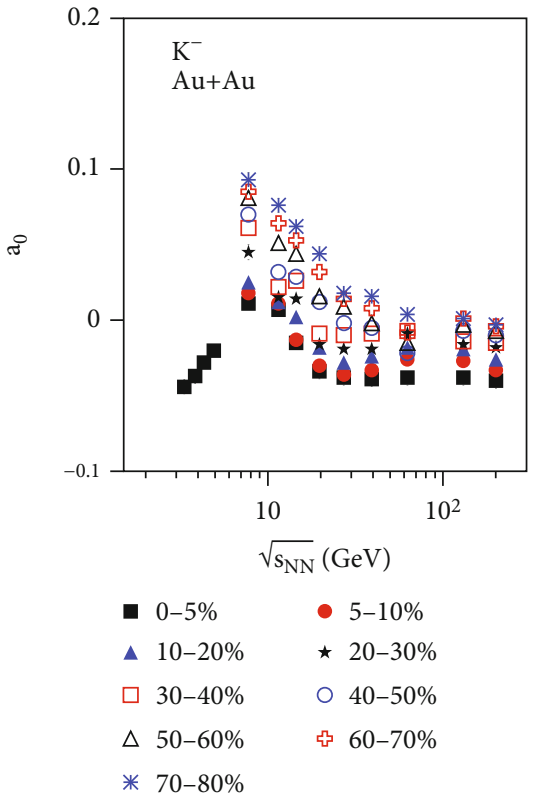

(c)

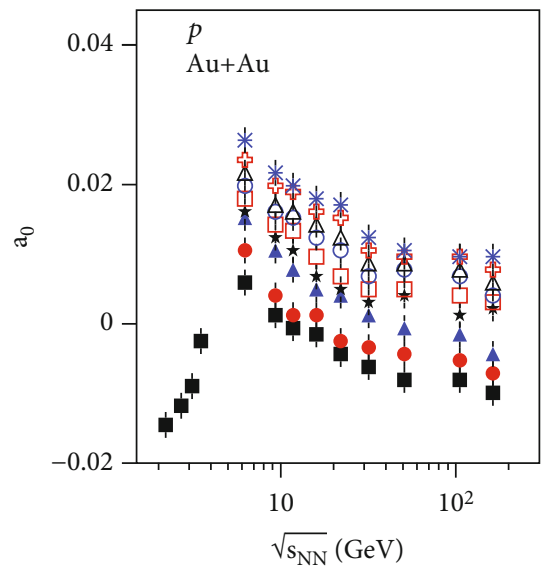
- $0-5 \%$
- $5-10 \%$
- $10-20 \%$
^ $20-30 \%$
$\square \quad 30-40 \%$
○ $40-50 \%$
$\triangle 50-60 \%$
њ $60-70 \%$

(d)

e)

(f)

FIgURE 13: Same as Figure 11, but showing the dependence of correction index $a_{0}$ on $\sqrt{s_{N N}}$. 


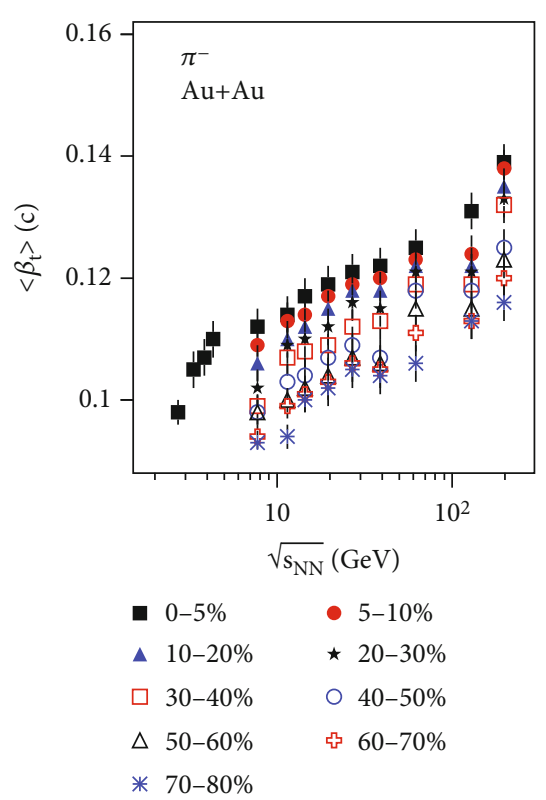

(a)

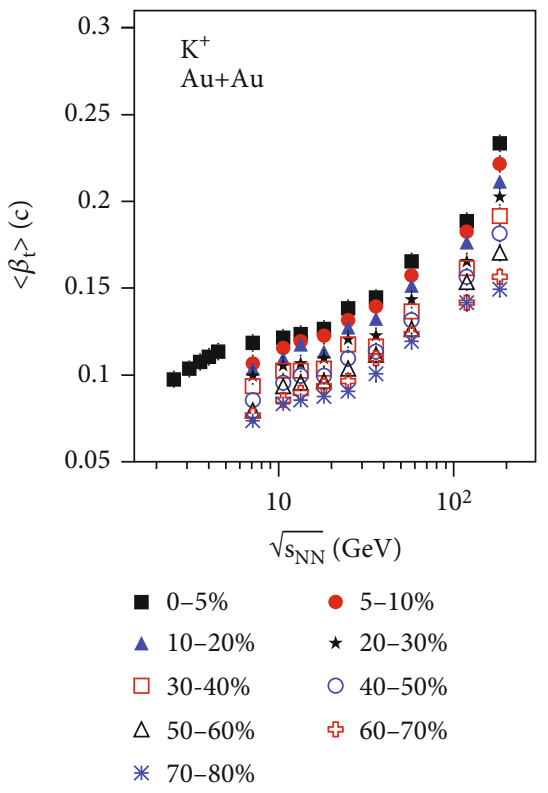

(d)

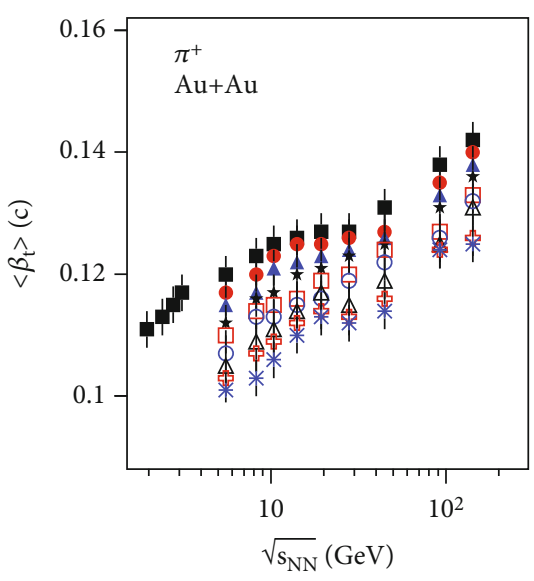

- $0-5 \%$ - $5-10 \%$

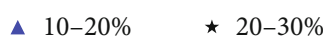

$\square 30-40 \% \quad \bigcirc \quad 40-50 \%$

$\triangle 50-60 \%$ ↔ $60-70 \%$

* $70-80 \%$

(b)

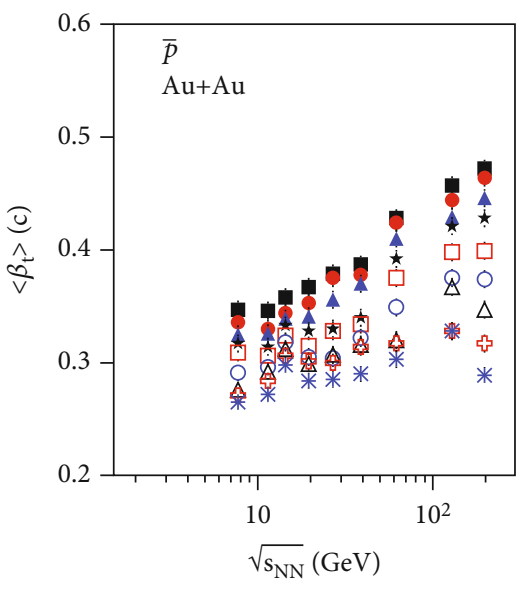

$\begin{array}{ll}\text { - } 0-5 \% & \text { - } 5-10 \% \\ \text { \ } 10-20 \% & \star 20-30 \% \\ \square 30-40 \% & \circ 40-50 \% \\ \triangle 50-60 \% & \text { ↔ } 60-70 \% \\ \text { * } 70-80 \% & \end{array}$

(e)

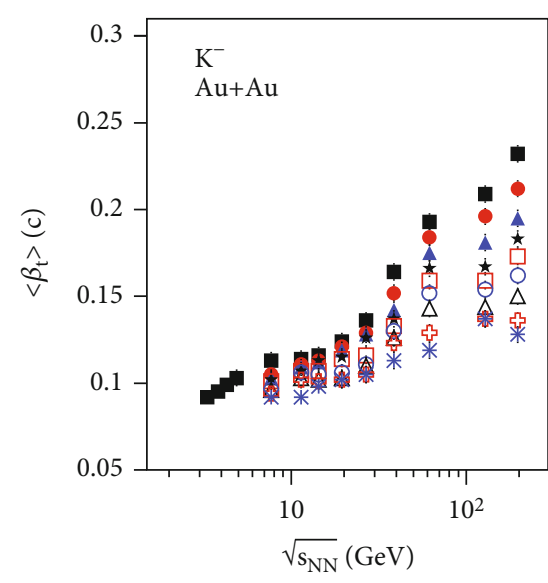

- $0-5 \%$ - $5-10 \%$

^ $10-20 \% \quad \star 20-30 \%$

$30-40 \% \quad \bigcirc \quad 40-50 \%$

$\triangle 50-60 \%$ ↔ $60-70 \%$

* $70-80 \%$

(c)

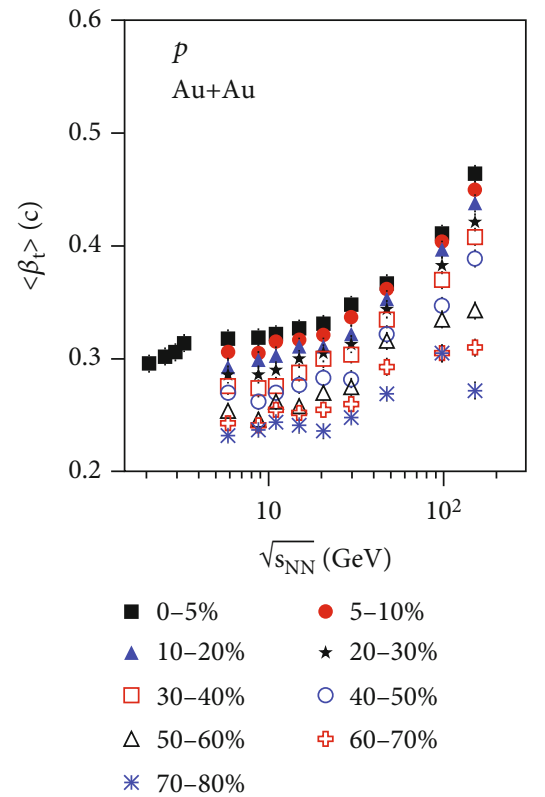

(f)

FIGURE 14: Same as Figure 11, but showing the dependence of average transverse flow velocity $\left\langle\beta_{t}\right\rangle$ on $\sqrt{s_{N N}}$. 


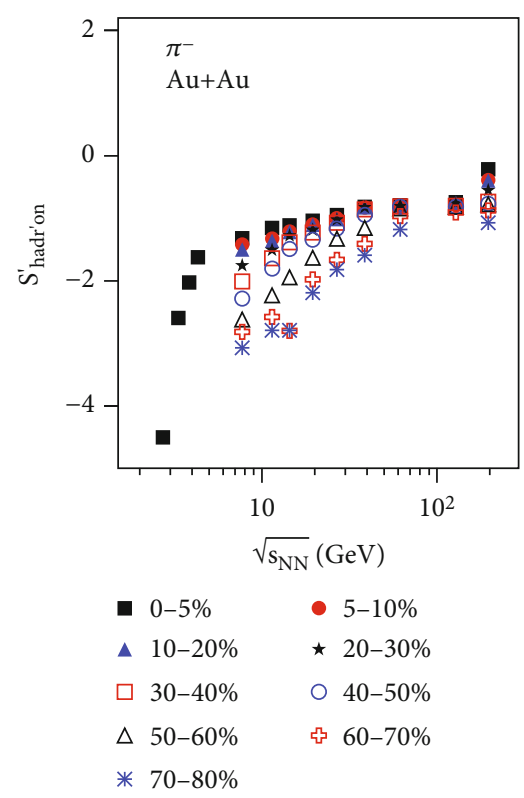

(a)

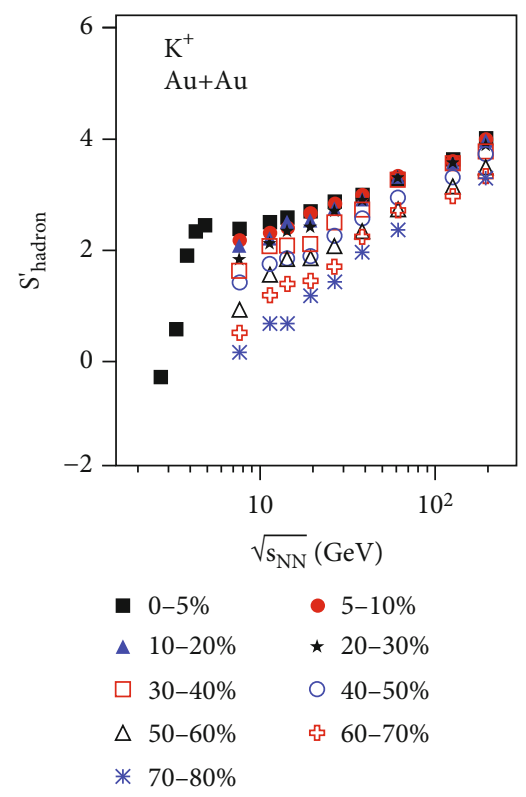

(d)

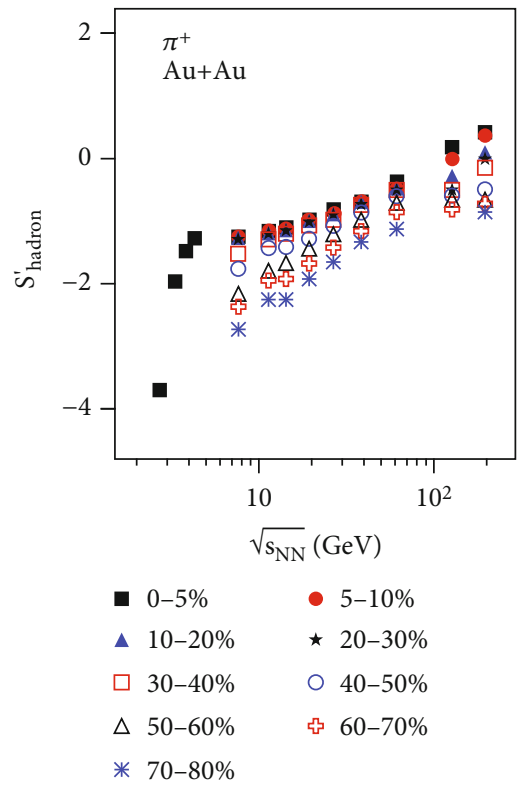

(b)

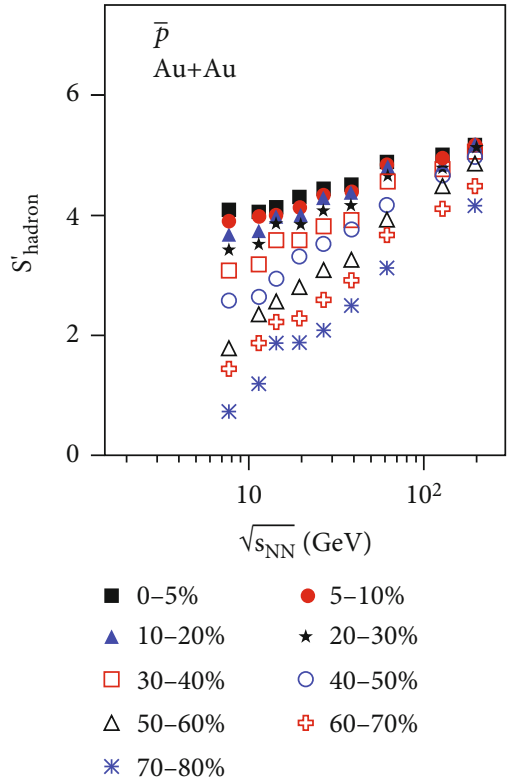

(e)

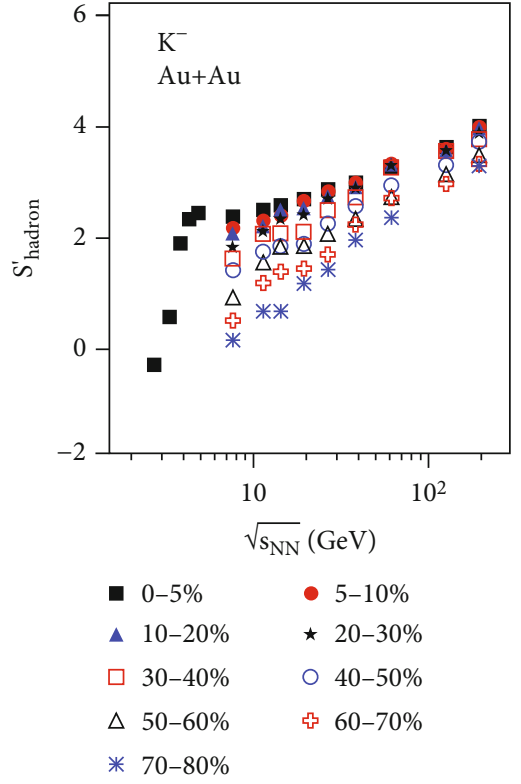

(c)

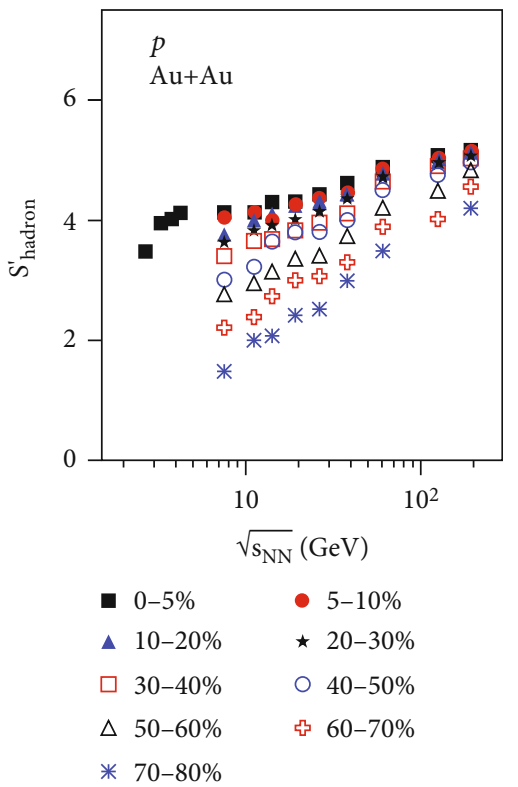

(f)

Figure 15: Same as Figure 11, but showing the dependence of pseudoentropy $S_{\text {hadron }}^{\prime}$ on $C$.

spectra in central Au-Au collisions increases quickly in the range of less than $7.7 \mathrm{GeV}$ and slowly in the range of greater than $7.7 \mathrm{GeV}$. Meanwhile, $\left\langle\beta_{t}\right\rangle$ increases with the increase of collision energy. From central to peripheral collisions, all of $n, T_{0}$, and $\left\langle\beta_{t}\right\rangle$ decrease, and $a_{0}$ increases. Even in peripheral collisions, the system is approximately at the equilibrium.

We have introduced the concept and definition of a new quantity, the pseudoentropy. The pseudoentropy is carried out a more in-depth study on the transverse momentum spectra of charged particles. With the increase of collision energy, the pseudoentropy extracted from the meson spectra in central Au-Au collisions increases quickly in the range of less than $7.7 \mathrm{GeV}$ and slowly in the range of greater than
7.7 GeV. This behavior is similar to that for $T_{0}$. We argue that $7.7 \mathrm{GeV}$ is a special energy at which the phase transition of deconfinement from hadronic matter to QGP can possibly happen. At least, the interaction mechanism in the system or the production mechanism of charged particles at the energy below and above about $7.7 \mathrm{GeV}$ are different.

\section{Data Availability}

The data used to support the findings of this study are included within the article and are cited at relevant places within the text as references. 


\section{Ethical Approval}

The authors declare that they are in compliance with ethical standards regarding the content of this paper.

\section{Disclosure}

The funding agencies have no role in the design of the study; in the collection, analysis, or interpretation of the data; in the writing of the manuscript; or in the decision to publish the results.

\section{Conflicts of Interest}

The authors declare that there are no conflicts of interest regarding the publication of this paper.

\section{Acknowledgments}

A preprint has previously been published [71]. The work of X.H.Z. and F.H.L. was supported by the National Natural Science Foundation of China under grant nos. 12147215, 12047571, 11575103, and 11947418; the Scientific and Technological Innovation Programs of Higher Education Institutions in Shanxi (STIP) under grant no. 201802017; the Shanxi Provincial Natural Science Foundation under grant no. 201901D111043; and the Fund for Shanxi "1331 Project" Key Subjects Construction. The work of X.H.Z. was also supported by the Innovative Foundation for Graduate Education in Shanxi University. The work of Y.Q.G. was supported by the Scientific and Technological Innovation Programs of Higher Education Institutions in Shanxi (STIP) under grant no. 2019L0629. The work of K.K.O. was supported by the Ministry of Innovative Development of the Republic of Uzbekistan within the fundamental project no. F3-20200929146 on analysis of open data on heavy-ion collisions at RHIC and LHC.

\section{References}

[1] S. Badger, J. M. Campbell, and R. K. Ellis, “QCD corrections to the hadronic production of a heavy quark pair and a W-boson including decay correlations," Journal of High Energy Physics, vol. 2011, no. 3, article 27, 2011.

[2] J. Gao, C. S. Li, B. H. Li, H. X. Zhu, and C.-P. Yuan, "Next-toleading order QCD corrections to a heavy resonance production and decay into top quark pair at the LHC," Physical Review D, vol. 82, no. 1, article 014020, 2010.

[3] D. J. Gross, "The discovery of asymptotic freedom and the emergence of QCD," Proceedings of the National Academy of Sciences of the United States of America, vol. 102, no. 26, pp. 9099-9108, 2005.

[4] A. Khuntia, S. Tripathy, R. Sahoo, and J. Cleymans, "Multiplicity dependence of non-extensive parameters for strange and multi-strange particles in proton-proton collisions at $\sqrt{s}=7 \mathrm{TeV}$ at the LHC," The European Physical Journal A, vol. 53, no. 5, p. 103, 2017.

[5] H. Satz and R. Stock, "Quark matter: the beginning," Nuclear Physics A, vol. 956, pp. 898-901, 2016.

[6] S. Furusawa, T. Sanada, and S. Yamada, "Hydrodynamical study on the conversion of hadronic matter to quark matter:
II. Diffusion-induced conversion," Physical Review D, vol. 93, no. 4, article 043019, 2016.

[7] S. Imai, H. Toki, and W. Weise, "Quark-hadron matter at finite temperature and density in a two-color PNJL model," Nuclear Physics A, vol. 913, pp. 71-102, 2013.

[8] B. Liu, M. D. Toro, G. Y. Shao, V. Greco, C. W. Shen, and Z. H. $\mathrm{Li}$, "Hadron-quark phase coexistence in a hybrid MIT-Bag model," The European Physical Journal A, vol. 47, no. 9, article 104, 2011.

[9] B. Mohanty, "Exploring the quantum chromodynamics landscape with high-energy nuclear collisions," New Journal of Physics, vol. 13, no. 6, article 065031, 2011.

[10] P. Braun-Munzinger and J. Wambach, "Colloquium: phase diagram of strongly interacting matter," Reviews of Modern Physics, vol. 81, no. 3, pp. 1031-1050, 2009.

[11] K. Fukushima and T. Hatsuda, "The phase diagram of dense QCD," Reports on Progress in Physics, vol. 74, no. 1, article 014001, 2011.

[12] N. Bratovic, T. Hatsuda, and W. Weise, "Role of vector interaction and axial anomaly in the PNJL modeling of the QCD phase diagram," Physics Letters B, vol. 719, no. 1-3, pp. 131135,2013

[13] S. Carignano, D. Nickel, and M. Buballa, "Influence of vector interaction and Polyakov loop dynamics on inhomogeneous chiral symmetry breaking phases," Physical Review D, vol. 82, no. 5, article 054009, 2010.

[14] L. Adamczyk, J. R. Adams, J. K. Adkins et al., "Collision energy dependence of moments of net-kaon multiplicity distributions at RHIC," Physics Letters B, vol. 785, pp. 551-560, 2018.

[15] L. Adamczyk, J. K. Adkins, G. Agakishiev et al., "Energy dependence of moments of net-proton multiplicity distributions at RHIC," Physical Review Letters, vol. 112, no. 3, article 032302, 2014.

[16] L. Adamczyk, J. K. Adkins, G. Agakishiev et al., "Beam-energy dependence of the directed flow of protons, antiprotons, and pions in Au+Au collisions," Physical Review Letters, vol. 112, no. 16, article 162301, 2014.

[17] L. Adamczyk, J. K. Adkins, G. Agakishiev et al., "Beam energy dependence of moments of the net-charge multiplicity distributions in $\mathrm{Au}+\mathrm{Au}$ collisions at RHIC," Physical Review Letters, vol. 113, no. 9, article 092301, 2014.

[18] X. F. Luo, "Exploring the QCD phase structure with beam energy scan in heavy-ion collisions," Nuclear Physics A, vol. 956, pp. 75-82, 2016.

[19] S. Ejiri, R. Iwami, and N. Yamada, "Critical point search from an extended parameter space of lattice QCD at finite temperature and density," Nuclear Physics A, vol. 956, pp. 826-829, 2016.

[20] I. Arsene, I. G. Bearden, D. Beavis et al., "Quark-gluon plasma and color glass condensate at RHIC? The perspective from the BRAHMS experiment," Nuclear Physics A, vol. 757, no. 1-2, pp. 1-27, 2005.

[21] K. Adcox, S. S. Adler, S. Afanasiev et al., "Formation of dense partonic matter in relativistic nucleus-nucleus collisions at RHIC: experimental evaluation by the PHENIX Collaboration," Nuclear Physics A, vol. 757, no. 1-2, pp. 184-283, 2005.

[22] B. B. Back, M. D. Baker, M. Ballintijn et al., "The PHOBOS perspective on discoveries at RHIC," Nuclear Physics A, vol. 757, no. 1-2, pp. 28-101, 2005.

[23] J. Adams, M. M. Aggarwal, Z. Ahammed et al., "Experimental and theoretical challenges in the search for the quark-gluon 
plasma: the STAR Collaboration's critical assessment of the evidence from RHIC collisions," Nuclear Physics A, vol. 757, no. 1-2, pp. 102-183, 2005.

[24] L. Kumar and for the STAR Collaboration, "Bulk properties in $\mathrm{Au}+\mathrm{Au}$ collisions at $\sqrt{\mathrm{s}_{\mathrm{NN}}}=9.2 \mathrm{GeV}$ in STAR experiment at RHIC," Nuclear Physics A, vol. 830, no. 1-4, pp. 275c-278c, 2009.

[25] B. Mohanty, "QCD phase diagram: phase transition, critical point and fluctuations," Nuclear Physics A, vol. 830, no. 1-4, pp. 899c-907c, 2009.

[26] C. Yang and for the STAR Collaboration, "The STAR detector upgrades and physics in beam energy scan phase II," EPJ Web of Conferences, vol. 182, article 02130, 2018.

[27] K. C. Meehan and for the STAR Collaboration, "Fixed target collisions at STAR," Nuclear Physics A, vol. 956, pp. 878-881, 2016.

[28] X. Sun and for the STAR Collaboration, "Flow in the RHIC beam energy scan from STAR," Journal of Physics: Conference Series, vol. 535, article 012005, 2014.

[29] K. C. Meehan and for the STAR Collaboration, "The fixedtarget experiment at STAR," Journal of Physics: Conference Series, vol. 742, article 012022, 2016.

[30] G. Odyniec, "The RHIC beam energy scan program in STAR and what's next...," Journal of Physics: Conference Series, vol. 455, article 012037, 2013.

[31] J. Cleymans, H. Oeschler, K. Redlich, and S. Wheaton, "Comparison of chemical freeze-out criteria in heavy-ion collisions," Physical Review C, vol. 73, no. 3, article 034905, 2006.

[32] C. Yang and for the STAR Collaboration, "The STAR beam energy scan phase II physics and upgrades," Nuclear Physics A, vol. 967, pp. 800-803, 2017.

[33] L. Adamczyk, J. K. Adkins, G. Agakishiev et al., "Bulk properties of the medium produced in relativistic heavy-ion collisions from the beam energy scan program," Physical Review C, vol. 96, no. 4, article 044904, 2017.

[34] J. Adam, L. Adamczyk, J. R. Adams et al., "Bulk properties of the system formed in $\mathrm{Au}+\mathrm{Au}$ collisions at $\sqrt{\mathrm{s}_{\mathrm{NN}}}=14.5 \mathrm{GeV}$ at the BNL STAR detector," Physical Review C, vol. 101, no. 2, article 024905, 2020.

[35] B. I. Abelev, M. M. Aggarwal, Z. Ahammed et al., "Systematic measurements of identified particle spectra in pp, d+Au, and $\mathrm{Au}+\mathrm{Au}$ collisions at the STAR detector," Physical Review C, vol. 79, no. 3, article 034909, 2009.

[36] J. L. Klay, N. N. Ajitanand, J. M. Alexander et al., "Charged pion production in $2 \mathrm{~A}$ to $8 \mathrm{~A} \mathrm{GeV}$ central $\mathrm{Au}+\mathrm{Au}$ collisions," Physical Review C, vol. 68, no. 5, article 054905, 2003.

[37] L. Ahle, Y. Akiba, K. Ashktorab et al., "An excitation function of $\mathrm{K}^{-}$and $\mathrm{K}^{+}$production in $\mathrm{Au}+\mathrm{Au}$ reactions at the AGS," Physics Letters B, vol. 490, no. 1, pp. 53-60, 2000.

[38] J. L. Klay, N. N. Ajitanand, J. M. Alexander et al., "Longitudinal flow of protons from (2-8)A GeV central Au+Au collisions," Physical Review Letters, vol. 88, no. 10, article 102301, 2002.

[39] C. Tsallis, "Possible generalization of Boltzmann-Gibbs statistics," Journal of Statistical Physics, vol. 52, no. 1-2, pp. 479487, 1988.

[40] T. S. Biró, G. Purcsel, and K. Ürmössy, "Non-extensive approach to quark matter," The European Physical Journal A, vol. 40, no. 3, pp. 325-340, 2009.

[41] H. Zheng and L. L. Zhu, "Can Tsallis distribution fit all the particle spectra produced at RHIC and LHC?," Advances in High Energy Physics, vol. 2015, Article ID 180491, 9 pages, 2015.
[42] H. Zheng, L. L. Zhu, and A. Bonasera, "Systematic analysis of hadron spectra in $\mathrm{p}+\mathrm{p}$ collisions using Tsallis distributions," Physical Review D, vol. 92, no. 7, article 074009, 2015.

[43] S. Chatrchyan, V. Khachatryan, A. M. Sirunyan et al., "Study of the inclusive production of charged pions, kaons, and protons in pp collisions at $\sqrt{s}=0.9,2.76$, and $7 \mathrm{TeV}$," The European Physical Journal C, vol. 72, no. 10, article 2164, 2012.

[44] J. Cleymans and M. W. Paradza, "Tsallis statistics in high energy physics: chemical and thermal freeze-outs," Physics, vol. 2, no. 4, pp. 654-664, 2020.

[45] P.-P. Yang, F.-H. Liu, and R. Sahoo, "A new description of transverse momentum spectra of identified particles produced in proton-proton collisions at high energies," Advances in High Energy Physics, vol. 2020, Article ID 6742578, 16 pages, 2020.

[46] Y.-M. Tai, P.-P. Yang, and F.-H. Liu, "An analysis of transverse momentum spectra of various jets produced in high energy collisions," Advances in High Energy Physics, vol. 2021, Article ID 8832892, 16 pages, 2021.

[47] E. Schnedermann, J. Sollfrank, and U. Heinz, "Thermal phenomenology of hadrons from $200 \mathrm{~A} \mathrm{GeV} \mathrm{S+S} \mathrm{collisions,"} \mathrm{Phys-}$ ical Revies C, vol. 48, no. 5, pp. 2462-2475, 1993.

[48] M. Waqas and B.-C. Li, "Kinetic freeze-out temperature and transverse flow velocity in $\mathrm{Au}-\mathrm{Au}$ collisions at RHIC-BES energies," Advanves in High Energy Physics, vol. 2020, article 1787183, 14 pages, 2020.

[49] P. K. Khandai, P. Sett, P. Shukla, and V. Singh, "System size dependence of hadron $\mathrm{p}_{\mathrm{T}}$ spectra in $\mathrm{p}+\mathrm{p}$ and $\mathrm{Au}+\mathrm{Au}$ collisions at $\sqrt{\mathrm{s}_{\mathrm{NN}}}=200 \mathrm{GeV}$," Journal of Physics G, vol. 41, no. 2, article 025105, 2014.

[50] Z.-J. Xiao and C.-D. Lü, Introduction to Particle Physics, Science Press, Beijing, China, 2016.

[51] V. Andreev, A. Baghdasaryan, A. Baty et al., "Measurement of charged particle multiplicity distributions in DIS at HERA and its implication to entanglement entropy of partons," The European Physical Journal C, vol. 81, no. 3, article 212, 2021.

[52] X. Feal, C. Pajares, and R. A. Vazquez, "Thermal behavior and entanglement in $\mathrm{Pb}-\mathrm{Pb}$ and $p$-p collisions," Physical Review $C$, vol. 99, no. 1, article 015205, 2019.

[53] Z. Tu, D. E. Kharzeev, and T. Ullrich, "Einstein-PodolskyRosen paradox and quantum entanglement at subnucleonic scales," Physical Review Letters, vol. 124, no. 6, article 062001, 2020.

[54] D. E. Kharzeev and E. M. Levin, "Deep inelastic scattering as a probe of entanglement," Physical Review D, vol. 95, no. 11, article 114008, 2017.

[55] A. Motornenko, V. Vovchenko, C. Greiner, and H. Stoecker, "Kinetic freeze-out temperature from yields of short-lived resonances," Physical Review C, vol. 102, no. 2, article 024909, 2020.

[56] J. A. L. López and on behalf of the ATLAS Collaboration, "Measurement of charmonium production in heavy-ion collisions with the ATLAS detector," Nuclear Physics A, vol. 967, pp. 584-587, 2017.

[57] G. R. Che, J. B. Gu, W. C. Zhang, and H. Zheng, "Identified particle spectra in $\mathrm{Pb}-\mathrm{Pb}, \mathrm{Xe}-\mathrm{Xe}$ and $\mathrm{p}-\mathrm{Pb}$ collisions with the Tsallis blast-wave model," Journal of Physics G, vol. 48, no. 9, article 095103, 2021.

[58] A. Jaiswal, N. Haque, A. Abhishek et al., "Dynamics of QCD matter - current status," International Journal of Modern Physics E, vol. 30, no. 2, article 2130001, 2021. 
[59] J. Chen, J. Deng, Z. B. Tang, Z. B. Xu, and L. Yi, "Nonequilibrium kinetic freeze-out properties in relativistic heavy ion collisions from energies employed at the RHIC beam energy scan to those available at the LHC," Physical Review C, vol. 104, no. 3, article 034901, 2021.

[60] X. F. Luo, S. S. Shi, N. Xu, and Y. F. Zhang, "A study of properties of the QCD phase diagram in high-energy nuclear collisions," Particles, vol. 3, no. 2, pp. 278-307, 2020.

[61] S. Bhattacharyya, "Backward shower particle production in high energy nucleus-nucleus collisions - an outlook to centrality dependence," International Journal of Modern Physics E, vol. 30, no. 4, article 2150032, 2021.

[62] S. Chatterjee, S. Das, L. Kumar et al., "Freeze-out parameters in heavy-ion collisions at AGS, SPS, RHIC, and LHC energies," Advances in High Energy Physics, vol. 2015, Article ID 349013, 20 pages, 2015.

[63] A. Bacchetta, F. Delcarro, C. Pisano, M. Radici, and A. Signori, "Extraction of partonic transverse momentum distributions from semi-inclusive deep-inelastic scattering, Drell-Yan and Z-boson production," Journal of High Energy Physics, vol. 2017, no. 6, article 81, 2017.

[64] V. V. Anisovich, S. M. Gerasyuta, and A. V. Sarantsev, "Lowlying meson spectroscopy and confinement," International Journal of Modern Physics A, vol. 6, no. 4, pp. 625-666, 1991.

[65] L. Adamczyk, J. K. Adkins, G. Agakishiev et al., "Global $\Lambda$ hyperon polarization in nuclear collisions," Nature, vol. 548, pp. 62-65, 2017.

[66] S. A. Bass, M. Gyulassy, H. Stöcker, and W. Greiner, "Signatures of quark-gluon plasma formation in high energy heavyion collisions: a critical review," Journal of Physics G, vol. 25, no. 3, pp. R1-R57, 1999.

[67] D. Stauffer, "Scaling theory of percolation clusters," Physics Reports, vol. 54, no. 1, pp. 1-74, 1979.

[68] W. Busza, K. Rajagopal, and W. van der Schee, "Heavy ion collisions: the big picture and the big questions," Annual Review of Nuclear and Particle Science, vol. 68, pp. 339-376, 2018.

[69] W.-J. Xie and F.-S. Zhang, "Nuclear collective flows as a probe to the neutron-proton effective mass splitting," Physics Letters $B$, vol. 735 , no. 30, pp. 250-255, 2014.

[70] D. E. Kharzeev, J. Liao, S. A. Voloshin, and G. Wang, "Chiral magnetic and vortical effects in high-energy nuclear collisions-A status report," Progress in Particle and Nuclear Physics, vol. 88, pp. 1-28, 2016.

[71] X.-H. Zhang, Y.-Q. Gao, F.-H. Liu, and K. K. Olimov, "Thermal freeze-out parameters and pseudo-entropy from charged hadron spectra in high energy collisions," 2021, https://arxiv .org/abs/2112.09473. 